\title{
SIMULATION OF DAMAGE VIBRATION RESPONSE OF STRUCTURES WITH REALISTIC FIELD UNCERTAINTIES
}

\author{
A thesis submitted to \\ the Faculty of Graduate and Postdoctoral Affairs \\ in Partial Fulfillment of the requirements for the degree \\ Master of Applied Science \\ by \\ Nabeel Y. Khan \\ B.Sc. Civil Engineering \\ University of Kashmir, India \\ Department of Civil and Environmental Engineering \\ Carleton University \\ Ottawa-Carleton Institute of Civil and Environmental Engineering \\ September 2018 \\ (C)2018 Nabeel Khan
}




\begin{abstract}
The concept of Pseudo load is introduced and explained in this thesis. A methodology based on Pseudo load has been developed that can reliably simulate the damage response of structures with realistic noise and uncertainties observed in the field. The method is applied and tested on numerical examples with field captured vibration data having realistic noise and uncertainties as well as on random data. It is shown that the Pseudo load method is effective in preserving the influences of noise and uncertainties in the simulated damage vibration response of the example structures. The limitations in the developed Pseudo load methodology are identified and resolved. The methodology is implemented on the Confederation Bridge to obtain simulated field damage responses of the bridge for further research on the development of vibration based structural health monitoring algorithms and tools.
\end{abstract}




\section{Acknowledgements}

I am thankful to Almighty Allah for the countless blessing and wisdom he bestowed upon me, the strength, the peace of mind and good health in order to finish this Research.

This thesis became a reality with the kind support and help of many individuals and institutions. I would like to sincerely thank all of them

To my supervisors: Dr. David T. Lau and Dr. Heng A. Khoo for their time, guidance, and unwavering support, for the quality knowledge they shared with me, for their advice and thoughtful observations in improving my work, for the insight they instilled in me. I am extremely fortunate to have the opportunity to learn and grow under their supervision. I owe special thanks to professor David T. Lau for accepting me as his research assistant and giving me the opportunity to work on Confederation Bridge monitoring project.

To my thesis committee: Dr. Murat Saatcioglu and Dr. Abhijit Sarkar for their insightful comments and encouragement.

To the Faculty of Graduate Studies at Carleton University for the Teaching Assistantship and the Department of Civil and Environmental Engineering for awarding me Kochar Family Scholarship 2016-2018 to pursue my graduate studies.

To Mr. and Mrs. Kochar for their generous funding to Kochar family scholarship, of which I am a grateful recipient. 
To my family for their valuable support and continuous encouragement. Words cannot express how grateful I am to my lovely mother, Zahida for her love, patience, sacrifice and support during my entire life. Your prayers have sustained me this far.

Finally, I would like to thank all my friends and colleagues especially Haseeb, Hissan, Mohannad and Tarundeep for sharing their knowledge, experiences and time with me, and for their constant support and motivation. 


\section{Table of Contents}

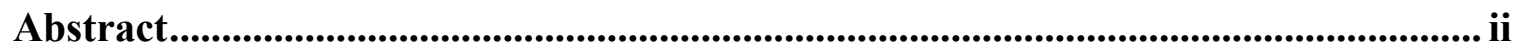

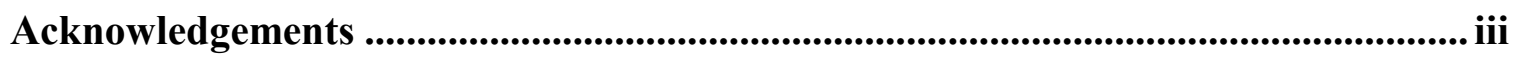

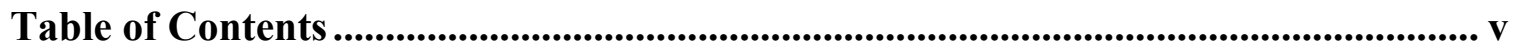

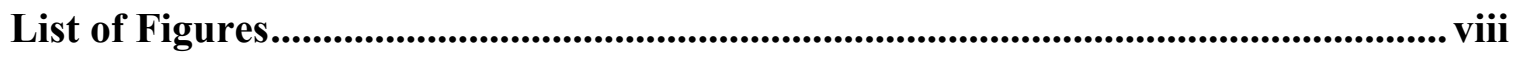

List of Tables ............................................................................................................................... xii

List of Symbols and Abbreviations .................................................................. xiii

Chapter 1: Introduction .............................................................................................................. 1

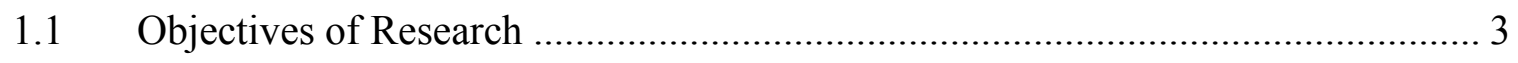

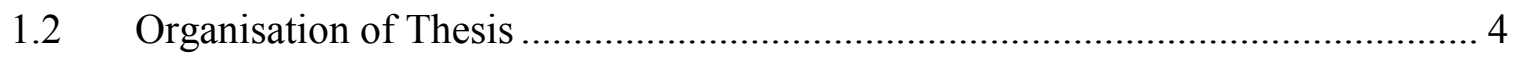

Chapter 2: Proposed Methodology and Validation .................................................... 5

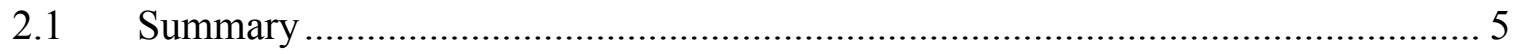

2.2 Introduction to Vibration based SHM (VBSHM) ......................................... 8

2.2.1 Effect of Operational and Environmental Variables...................................... 11

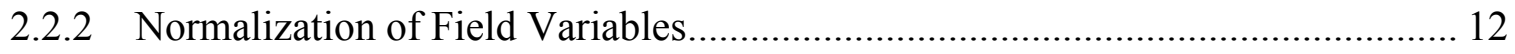

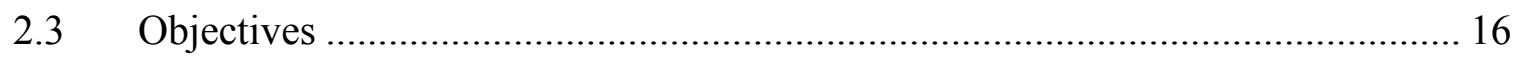

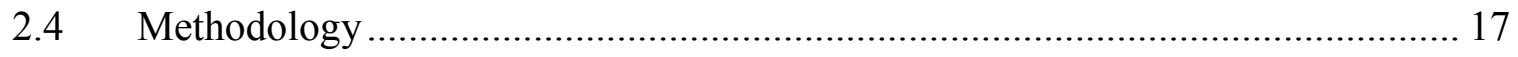

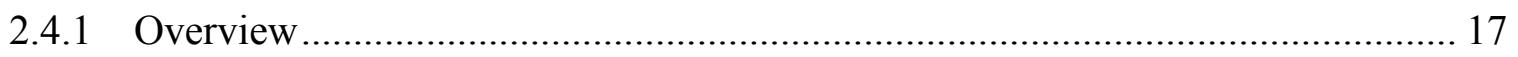

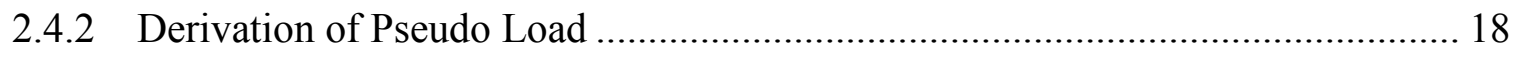

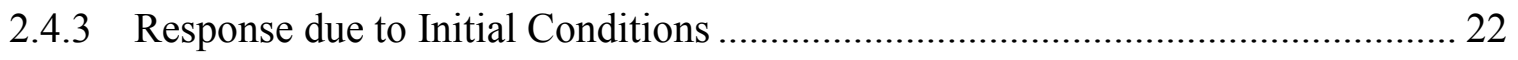

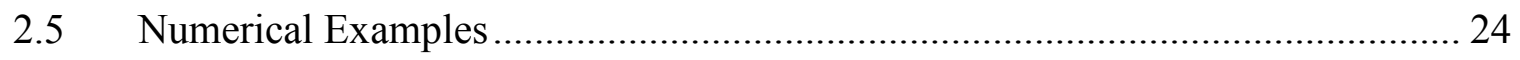




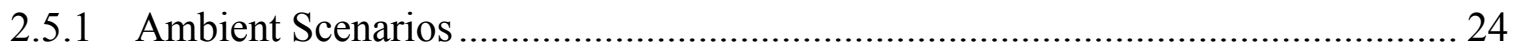

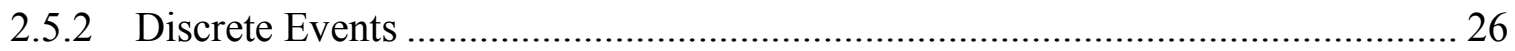

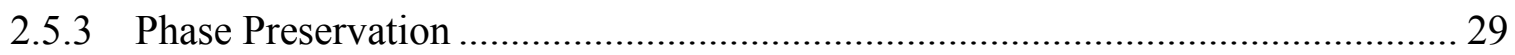

Chapter 3 Case Study of Simulation of Damage Vibration Response ......................... 53

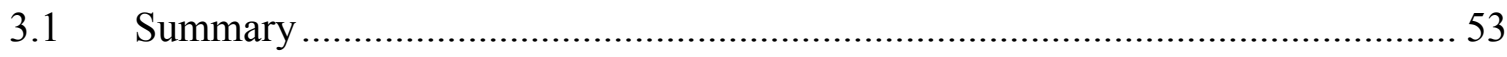

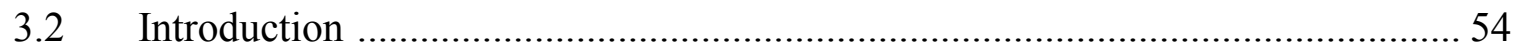

3.3 Vibration Monitoring of Confederation Bridge ................................................... 57

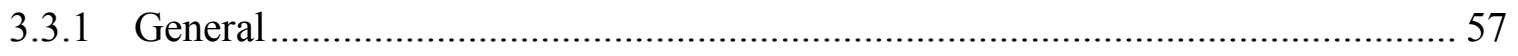

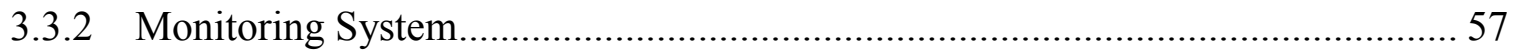

3.3.3 Data Processing by Graphic User Interface (GUI) ……………………............ 58

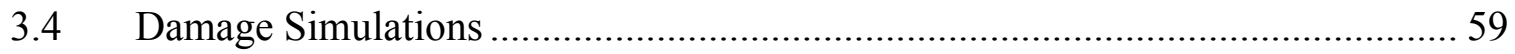

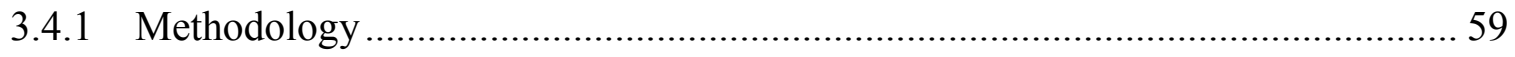

3.4.2 Pseudo Load for Monitored Structures ............................................................... 60

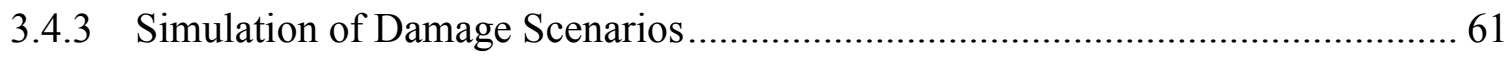

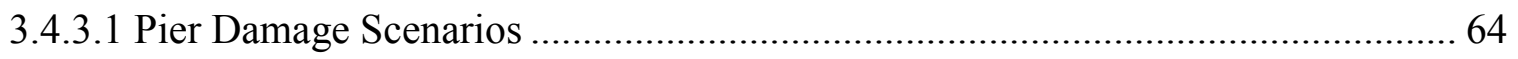

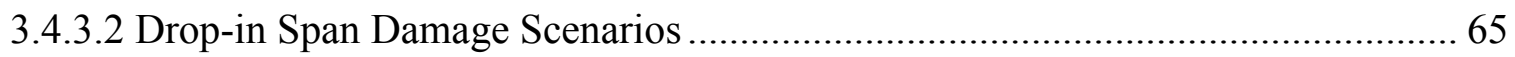

3.5 Discussion on Simulated Damage Responses.................................................... 65

Chapter 4 Conclusions and Recommendations.................................................................... 90

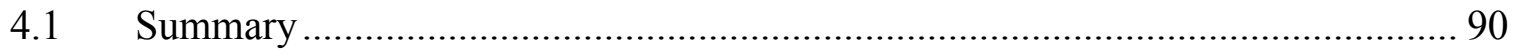

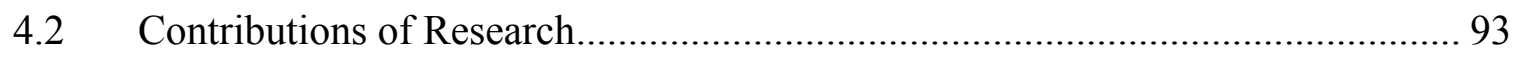

4.3 Conclusions and Future Works......................................................................... 94 


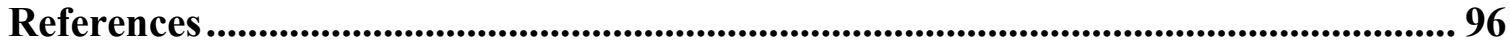




\section{List of Figures}

2.1 Basic focus of the research...............................................

2.2 The schematic diagram of how damage responses can be simulated from measured responses and finite element model...................................... 32

2.3 (a) How to compute Pseudo loads and (b) how Pseudo loads can be used to simulate damage responses................................................33

2.4 Structural model of the four storey moment resisting frame building example......34

2.5 Random loads for the example building ...................................

2.6 Acceleration responses of the example building ............................ 36

2.7 Percentage normalised error in the damage response simulated by Pseudo load at;

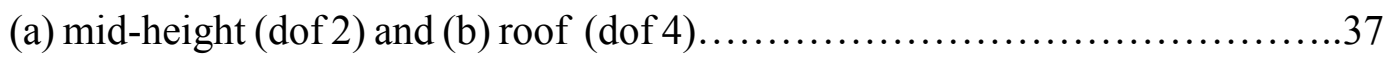

2.8 Picture of the building in Richmond, California with accelerometers installed at all floor levels, provided by The Centre of Engineering Strong Motion Data (CESMD) in website www.strongmotioncentre.org (b) simplified model adopted for

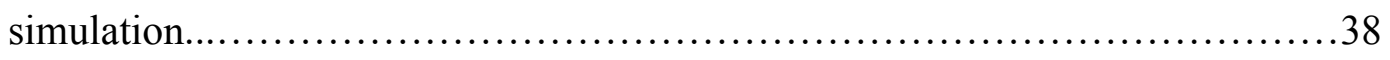

2.9 Acceleration response recorded by the sensors at the three floor levels in EW lateral direction of the building provided by The Centre of Engineering Strong Motion Data (CESMD) in website www.strongmotioncentre.org.

2.10 Percentage normalized error in the response simulated by Pseudo load at; (a) first floor (dof 1) and (b) roof (dof 3), of the model adopted in discrete event example..40

2.11 Normalized relative error in the response simulated by Pseudo load at; (a) first floor (dof 1) and b) roof (dof 3), of the model adopted in discrete event example.....41 
2.12 Comparison of the exact response and the response simulated by Pseudo load at first floor of the model adopted in discrete event example 42

2.13 Comparison of the exact response and the response simulated by Pseudo load at roof level of the model adopted in discrete event example 43

2.14 (a) Comparison of the exact phase and the phase obtained from response simulated by Pseudo load in the model adopted in discrete event example at first floor (b) difference between the exact phase and the phase obtained from response simulated by Pseudo load. 44

2.15 Normalized relative error in the response simulated by Pseudo load in the ambient scenario example at; (a) mid height (dof 2) and b) roof (dof 4) 45

2.16 Figure 2.17. Comparison of (a) the exact response and (b) the response simulated by Pseudo load at the roof level in the ambient scenario example 46

2.17 Comparison of the exact response and the response simulated by Pseudo load at the roof level in the ambient scenario event example.

2.18 (a) Comparison of the exact phase and the phase obtained from response simulated by Pseudo load in the ambient scenario example at roof level (b) difference between the exact phase and the phase obtained from response simulated by Pseudo load...48

3.1 (a) Dimensions and main component of typical structural module span (b) location of 50 accelerometers out of the total 76 installed in Confederation Bridge monitoring system

3.2 Visualization module of GUI developed for the Confederation Bridge taken from the SPLASH algorithm developed by Desjardins (2004). 68 
3.3 (a) Test structure of Confederation Bridge used for demonstrating the proposed methodology (b) beam element model of the test structure schematic showing mesh

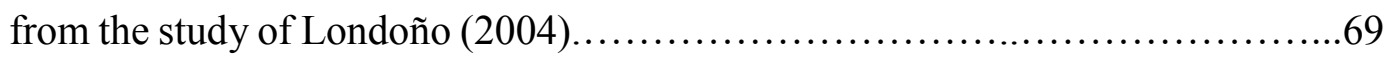

3.4 Transverse acceleration response recorded by sensors (a) 1 and (b) 3 for simulating damage response in pier damage scenario ........................................................ 70

3.5 Transverse acceleration response recorded by sensors (a) 8 and (b) 9 for simulating damage response in drop-in span joint damage scenario ....................................... 71

3.6 Normalized relative error in the response simulated by Pseudo load at sensor 1 for

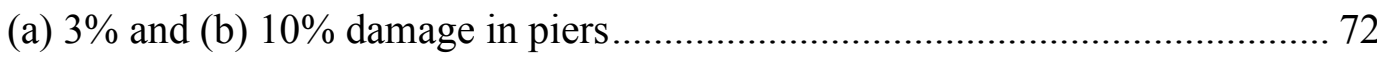

3.7 Comparison of the exact response and the response simulated by Pseudo load at sensor 1 for $3 \%$ damage in piers 73

3.8 Comparison of the exact response and the response simulated by Pseudo load at sensor 1 for $10 \%$ damage in piers 74

3.9 Comparison of the (a) exact phase and (b) the phase obtained from response simulated by Pseudo load at sensor 1 for $10 \%$ damage in piers that satisfies the criterion in Eq. 2.19, with (c) difference in phase results. 75

3.10 Normalized relative error in the response simulated by Pseudo load at sensor 8 for (a) $3 \%$ and (b) $10 \%$ damage in drop-in span joint 76

3.11 Comparison of the exact response and the response simulated by Pseudo load at sensor 8 for 3\% damage in drop-in span joint 77

3.12 Comparison of the exact response and the response simulated by Pseudo load at sensor 8 for $10 \%$ damage in drop-in span joint 78 
3.13 Comparison of the (a) exact phase and (b) the phase obtained from response simulated by Pseudo load at sensor 8 for $10 \%$ damage in drop-in span joint that satisfies the criterion in Eq. 2.19, with (c) difference in phase results 79

3.14 Comparison of the relative difference between the captured response and the response simulated by Pseudo load at sensor 1; (a) captured (b) difference with 3\% damage (c) difference with 5\% damage (d) difference with 10\% damage........ 80

3.15 Comparison of the relative difference between the captured response and the response simulated by Pseudo load at sensor 8; (a) captured (b) difference with 3\% damage (c) difference with 5\% damage (d) difference with $10 \%$ damage. 81

3.16 Comparison of the power spectral density of the (a) captured response and the response simulated by Pseudo load at sensor 1 for (b) 3\% and (c) 10\% damage in

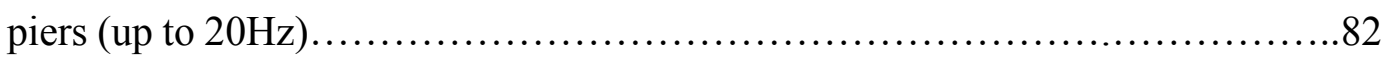

3.17 Power spectral density of the response captured by sensor $8 \ldots \ldots \ldots \ldots \ldots \ldots \ldots \ldots \ldots . . . . . \ldots 3$

3.18 Power spectral density of the response simulated by Pseudo load at sensor 1 for 3\%

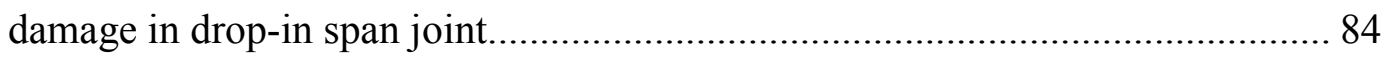

3.19 Power spectral density of the response simulated by Pseudo load at sensor 8 for $10 \%$ damage in drop-in span joint

3.20 Absolute difference between the power spectral densities of the captured response and the response simulated by Pseudo load at sensor 8 for 3\% damage in drop-in span joint. 86

3.21 Absolute difference between the power spectral densities of the captured response and the response simulated by Pseudo load at sensor 8 for $10 \%$ damage in drop-in span joint 87 


\section{List of Tables}

Table 2.1 Geometric and material properties of themodel in ambient scenario example...49

Table 2.2 Initial conditions in the ambient scenario example..........................50

Table 2.3 Geometric and material properties of the model in discrete event example......51

Table 2.4 Initial conditions in the discrete event example.........................52

Table 3.1 Initial conditions for pier damage simulation.............................. 88

Table 3.2 Initial conditions for drop in span joint damage simulation...................89 


\section{List of Symbols and Abbreviations}

C Damping of a sdof system

[C] Damping matrix of a mdof system

$F_{n} \quad$ Dynamic load at $n$ channels

$K \quad$ Stiffness of a sdof system

[K] Stiffness matrix of a mdof system

$\left[\mathrm{K}_{\mathrm{d}}\right] \quad$ Stiffness matrix of damaged mdof structure

M Mass of a sdof system

[M] Mass matrix of a mdof system

$\left[\mathrm{M}_{\mathrm{d}}\right] \quad$ Mass matrix of damaged mdof structure

$n \quad$ Number of channels/sensors

$m \quad$ Number of degrees of freedom

$P_{\text {pseudo }} \quad$ Pseudo load

$S_{n} \quad$ Vector of initial displacements

$S_{0} \quad$ Initial displacement

$\ddot{u}_{n} \quad$ Captured acceleration response at $n$ channels

$\dot{u}_{n} \quad$ Captured velocity response at $n$ channels

$\dot{\tilde{u}}_{n} \quad$ Calculated velocity response at $n$ channels with zero initial condition 
$u_{n} \quad$ Captured displacement response at $n$ channels

$\tilde{u}_{n} \quad$ Calculated displacement response at $n$ channels with zero initial displacement

$\ddot{u}_{n d} \quad$ Simulated damage acceleration response at $n$ channels

$\dot{u}_{n d} \quad$ Simulated damage velocity response at $n$ channels

$\dot{\tilde{u}}_{n d} \quad$ Simulated damage velocity response at $n$ channels with zero initial velocity

$u_{n d} \quad$ Simulated damage displacement response at $n$ channels

$\tilde{u}_{n d} \quad$ Simulated damage response at $n$ channels with zero initial displacement

$\ddot{u}_{i} \quad$ Acceleration due to initial conditions

$\dot{u}_{i} \quad$ Velocity due to initial conditions

$u_{i} \quad$ Displacement due to initial displacement

$\ddot{u}_{s} \quad$ Captured acceleration response at sensor locations

$\dot{\tilde{u}}_{s} \quad$ Calculated velocity response at sensor locations with zero initial velocity

$\tilde{u}_{s} \quad$ Calculated displacement at sensor locations with zero initial displacement

$\ddot{u}_{d} \quad$ Unknown acceleration response at locations where sensors are unavailable

$\dot{\tilde{u}}_{d} \quad$ Unknown velocity response at locations where sensors are unavailable

$\tilde{u}_{d} \quad$ Unknown displacement response at locations where sensors are unavailable

$V_{n} \quad$ Vector of initial velocities

$V_{0} \quad$ Initial velocity 
dof Degrees of freedom

mdof Multiple degrees of freedom

VBSHM Vibration based structural health monitoring 


\section{Chapter 1. Introduction}

The technique of structural health monitoring encapsulates all the methodologies developed and implemented to assess the conditions and quantify the performance of structures for safe operations, timely repair, and maintenance. In current operation and maintenance practice of civil engineering structures, periodic visual inspection is still the most common approach adopted as a first step in the established methodology to evaluate the safety, structural conditions and assessing the needs of repair and maintenance of structures. It is commonly recognized that there are significant limitations with the existing practice with regards to inspection of parts of a structure that are inaccessible or difficult to reach, consistency and reliability of inspection results as they are dependent on the

judgement and experience of the inspection personnel. More crucially, the inability of current visual inspection practice to detect deterioration or abnormality before they become visible on the structure's surface prevents the adoption of early remedial or mitigation measures that would reduce repair and maintenance costs and allow for better performance of the structure. For critical structures, the impacts of these limitations are even more amplified. In recognition that the continuous reliable service and high performance of infrastructures come to symbolize the quality of life in terms of safety, security and socioeconomic well-being of society, there is the need to improve the aforementioned approach in ensuring the safe operation and cost-effective and efficient maintenance of society's important infrastructure. With the rapid technology advances in recent years in areas of sensors and telecommunication technologies, and the merging of these new advancements with those in traditional structural engineering fields of structural modelling and analysis, structural dynamics and system identifications, vibration based structural health 
monitoring (VBSHM) has emerged as a promising new method for non-destructive evaluation of civil infrastructure. In structural condition assessment by VBSHM approach, vibration response data of the structure captured by sensors of monitoring systems are processed and analyzed by system identification algorithms to extract information on the vibration characteristics of the monitored structure, such as its modal properties of vibration frequencies, mode shapes and damping ratios. The basic premise of VBSHM for identifying structural deterioration or damage is that any changes observed in the extracted dynamic characteristics compared to a reference such as that when the structure is healthy or undamaged are considered as manifestations of damage or deterioration or change in the conditions of the structure. Although VBSHM methods have a strong theoretical basis, it is found difficult to apply VBSHM techniques to real world structures because of the noise and uncertainties in the field monitoring data (Londoño et al. 2013). Consequently, any observed changes in the field extracted dynamic characteristics of a monitored structure are the combined results of the variabilities in the ambient operational and environmental conditions as well as any damage in the structure. The source of noise and uncertainties in the monitoring data can be from the structure itself, such as uncertainties in its boundary conditions and structural and material properties, and/or from its operation and surrounding environment such as temperature, wind speed and direction, traffic conditions on the structure (in case of bridges) or nearby surroundings (in the case of buildings), as well as measurement noise from the monitoring sensors and data capturing instrumentation and equipment etc. It is important to recognize that captured vibration response data from the field inherently contain the actual effects of noises and uncertainties from the operational and environmental conditions as well as from the sensors and monitoring systems in the 
field. It is a challenge for VBSHM system identification methods to analyze field monitoring data with noise and uncertainties without adverse impact on the accuracy and reliability of their structural condition assessment or damage detection results. In other words, it is critically important for the VBSHM methodologies to be able to distinguish any observed discrepancies in the field extracted dynamic characteristics of a monitored structure caused by noise and uncertainties from those due to damage and deteriorations in the structure. This is a critical challenge that must be overcome before VBSHM methods can be realistically accepted for implementation in the field to solve real world problems. In order to be able to test the validity of existing VBSHM methods or to develop more reliable VBSHM methods, a methodology is critically needed to realistically simulate the effects of noise and uncertainties in vibration response data of a structure.

\subsection{Objectives of Research}

The objective of this research is to develop a reliable methodology that can accurately simulate field damage vibration response data of structures with the effects of noise and uncertainties under any damage scenarios of the structures. By applying the developed methodology, the actual captured vibration response data of a monitored structure still in the intact state or condition in the field is processed and the influences of the noise and uncertainties in the captured response data can be identified. Applying these identified captured effects of noise and uncertainties to any simulated damage state of the structure, realistic damaged vibration responses of the structure with the same effects of noise and uncertainties as in the original initial intact state of the structure can be derived which 
guarantees that the differences between these two pairs of datasets are due to the simulated damage alone. These recorded and simulated damage vibration response data sets can then be used to evaluate the applicability of existing vibration based damage detection algorithms in practical cases or to develop more advanced field damage detection VBSHM algorithms and tools.

\subsection{Organization of Thesis}

Chapter 2 presents an overview of vibration based structural health monitoring (VBSHM) and its advantages in comparison to the existing common practice of visual inspection based approach for structural risk assessment. The limitations of applying VBSHM in practical applications of actual structures in the field are presented with the focus on operational and environmental uncertainties. A new methodology of Pseudo load to simulate the damage vibration response of structures with realistic field uncertainties is presented. Numerical examples are presented to demonstrate the validity and accuracy of the proposed new method.

Chapter 3 presents the derivation of the Pseudo load method and its application to the case of a real structure in the field. A brief overview of the Confederation Bridge and its monitoring system that has been collecting vibration response data since its opening in 1997 are discussed. Simulated vibration responses of the example structure under actual field uncertainty conditions for difference damage scenarios are generated and discussed.

Chapter 4 presents a brief summary of the research findings, conclusions and recommendations. 


\section{Chapter 2. Proposed Methodology and Validation}

\subsection{Summary}

Data from vibration based structural health monitoring (VBSHM) have been used in structural condition assessment and damage detection applications of various civil engineering structures, especially on large complex structures including the Confederation Bridge in Canada. The basic premise of structural health assessment and damage detection by VBSHM is based on identifying the changes in structural dynamic response parameters quantified from vibration response data captured by sensors under ambient loading conditions, e.g. from wind and traffic, or extreme event loadings. In recent years, several damage detection algorithms based on VBSHM approach have been developed by many researchers worldwide. However, most of these early research and algorithms do not consider the influence of noise and uncertainties from the field (Kullaa 2003 and 2004, Sohn et al. 2003, Yan et al. 2005a, and Figueiredo et al. 2010). In reality, there are considerable uncertainties in the captured monitoring data of real structures under the field operational conditions which are difficult or near impossible to replicate in laboratory tests or by numerical models. To detect the damage of structural health and to arrest its deterioration at it earliest stage so that one can take remedial measures, it is necessary for the developed algorithms to have enough sensitivity to detect smallest possible change in the structural response parameters, which are highly sensitive to noise and uncertainties in the captured monitoring data. The noise and uncertainties in the monitoring data comes from measurement noise from sensor equipment and variability of the structural response and environmental conditions, such as boundary conditions, material properties, 
temperature, loading condition, wind speed and direction, traffic conditions etc. Although some existing damage detection algorithms account for the effect of uncertainties by considering white noise (He et al. 2014) with uniform power spectral density, however they do not reflect the realistic characteristics of actual uncertainties of structures exposed and operating in the field.

To address the issues of variabilities in field operational and environmental conditions and their effect on the recorded vibration data, researchers have developed algorithms that attempt to identify changes in the structural response parameters primarily due to structural damages. Some of these vibration based algorithms have been tested on data obtained from laboratory testing under controlled environment. But it is noted that controlled laboratory testing does not include the effect of field noise and uncertainties (Sohn 2003, Kullaa 2003 and 2004, Yan et al. 2005a, Figueiredo et al. 2010). Other researchers have developed statistical models to quantify noise and uncertainties and applied these statistical models to investigate the effect of noise and uncertainties on the variabilities and accuracy of the results of vibration based structural condition assessment and damage detection algorithms (Londoño and Lau 2003, Londoño et al. 2013). Some of these statistical models are developed by observing the correlation between selected operational and environmental parameters of temperature (Peeters 2000), wind, traffic, ambient and other load scenarios (Londoño et al. 2013, Li et al. 2018) and structural response in field captured monitoring data. However, civil engineering structures which are exposed to harsh environmental conditions like the Confederation Bridge in Canada can have noise and uncertainties in the captured responses from a wide range of sources which may be unknown. Each source of noise and uncertainty may affect the structural response parameters differently. Therefore, 
the statistical models and algorithms developed and tested using such data (Yan et al. 2005b, Kullaa 2014) would have limitation on practical applications. Also, because of the wide range of noise and uncertainties which civil engineering structures may be subjected to, it is difficult or impractical to develop comprehensive statistical models that can quantify all sources of noise and uncertainties and their effect on the vibration behaviour of monitored structures in the field. Not only that development of meaningful statistical models requires long term monitoring and continuous collection of vast amounts of response monitoring data, there are limitations on the applicability of these statistical models. While the statistical models developed based on observations extracted from data collected in the past can be updated with new data as they are collected, it is still difficult to use these statistical models to predict the characteristics of noise and uncertainties in the future due to the wide range of variabilities and unknown uncertainties in the operation of civil engineering structures in the field. Furthermore, it is important to recognize that the statistical models developed for one structure are not likely to be readily applicable to another structure because the noise and uncertainties related to the specific field operational and environmental conditions of one structure may not be the same in the case of another structure. Therefore, there is the need to overcome the limitations inherent in the statistical models and to find an alternate approach that not only can be used to simulate the damage response of monitored structures with realistic noise and uncertainties in the field, but also has the universal applicability to all monitored structures.

In the proposed methodology, actual field monitoring data are used as the basis for simulating the damage structure response data. Since the field monitoring data preserve all the noise and uncertainties of the structural response behaviour and environment 
conditions, as such they are the exact representation of the field noise and uncertainties. Any changes to the structural response due to change in the structural condition of finite element models are combined with the preserved noise and uncertainties in the monitoring data to give the realistic simulated vibration response data of the damaged structure. The proposed methodology duplicates the noise and uncertainties present in the field captured responses and applies them to the simulated damage response data of the monitored structure. The damage response generated therefore has the damage characteristics of the monitored structure and the realistic noise and uncertainties captured from the field. Such response data are needed to examine the effectiveness of existing vibration based damage detection algorithms especially when the structural response parameters are masked by field noise and uncertainties. At the same time, the proposed new methodology would also overcome the limitations of statistical model based VBSHM methods. Numerical examples are presented to demonstrate the capability and validity of the proposed method.

\subsection{Introduction to Vibration Based Structural Health Monitoring (VBSHM)}

With advanced technologies and invention of new materials, complex structures based on presumed field behaviours are designed and constructed worldwide. Owing to the great amount of effort in technologies, time and investment demanded to realize the construction of important structures, and to maintain our confidence in their continuous operation, it is important to continuously scrutinize the performance and keep up with the maintenance of these structures in order to maintain and prolong their functionality and service life. Furthermore, structures located in harsh environment of temperature, humidity and snow, 
and continuously subjected to extreme load effects of traffic, wind and earthquakes, could suffer progressive deterioration or sudden damage due to overstress from extreme accident or natural hazard events. The research of structural health monitoring (SHM) for civil engineering applications is the development and application of new technologies and methodologies to maintain the integrity and reliability of civil infrastructure. The goal of SHM is to detect, localize, and quantify structural damage and assess degradation rate to reduce the maintenance cost, rehabilitation time and chances of any catastrophic failure.

The traditional visual based approach of monitoring the performance of structures is highly subjective and dependent on the judgement and experience of the inspector. In addition, the accuracy of visual based structural inspection can be affected by issue of inaccessibility of some parts of the structure or structural components, e.g. submerged part of a structure. It is also difficult to detect initiation of damage at the initial phase of degradation, e.g. corrosion is often detected only after the damage has progressed for some time when the effect is finally shown up on the structure's surface. Sometimes the visual monitoring approach is aided by other destructive or non-destructive evaluation techniques like liquid penetration testing, magnetic particle testing, radiographic testing etc. to provide more detailed information on the condition of the inspected structure. However, these techniques need a priori knowledge of the location of damage and are thus not practical for applications, especially in large structures, to detect initiation of condition deterioration or structural damage. Additional limitations of these supplementary techniques include: limitation of material type that these techniques can be applied to, expensive, time consuming and cause disruption to the evaluated structure's operations. For critical structures with high social and economic importance the effects of the limitations are even 
more consequential. In light of these limitations, there is the need to improve the current practice and/or develop new approaches to ensure the safety and performance of society's infrastructure. Recently the field of vibration based structural health monitoring (VBSHM) has attracted increasing research attention and significant advances have been made in its development as an alternative to the current practice of structural evaluation, especially on its suitability for implementation in continuous monitoring systems for early detection of structural abnormalities.

In the most fundamental form though not restricted to, vibration based SHM techniques are based on detecting the changes in the dynamic characteristics of the structure such as natural frequencies, mode shapes and damping properties. As these characteristics are directly dependent on the geometric and material properties along with the boundary conditions of the structure, as such any change in the dynamic behaviour of the structure can be interpreted as an indication of possible change in the stiffness, mass or energy dissipation properties etc. of the structure. Consequently, the information of the detected change can be used to identify, locate, and quantify the severity of damage or change of condition in the structure. All VBSHM methodologies rely on response data measured using sensors, usually accelerometers, strain gauges etc., in time domain and if needed the data are converted into frequency domain and further into modal domain. Significant research has been done by many researchers worldwide to develop state-of-the-art damage detection methodologies and algorithms in all three domains, particularly in modal domain which has received prime attention because modal properties are efficient way with easy to understand physical meaning for characterizing the dynamic behaviour of a structural system. In the modal domain, any detected change in the modal properties of a structure 
can be interpreted as a change in the condition of the structure which can then be used to classify the structure into damaged or undamaged states. In comparison, the methodologies for condition assessment in time domain, by auto regressive models, or in frequency domain by frequency response functions, features are extracted from the vibration response data of the structure as characterization of the structure which may have the advantage of higher sensitivity to damage than modal properties in the modal domain.

\subsubsection{Effect of Operational and Environmental Variables}

In early research of VBSHM in quantification of the dynamic characteristics of a structure from its dynamic monitoring response, the influence of the environment on the dynamic behaviour of the structure is ignored. With recent advancements in VBSHM, the significance of the influence of variabilities in the field operational and environmental conditions on the dynamic characteristics of a structure is recognized (Rohrmann et al. 2000, Peeters et al. 2001, Sohn 2007, Hu et al. 2009, Kullaa 2010 and 2014, Westgate et al. 2011, Rahman and Lau 2013), and there are research efforts to quantify these influences due to operational conditions, such as ambient loading conditions, slight changes to the boundary conditions of the structure, and due to environmental conditions, such as temperature, humidity and wind speed and direction. Moorty and Roeder (1992) studied the effect of temperature on boundary conditions of Sutton Creek Bridge, in Kootenai Forest in Montana, and found significant expansion of the bridge deck with rise in temperature. Wood (1992) in his study on the effect of temperature on 5 bridges in UK found the stiffness changed due to change in temperature. Farrar et al. (1997) found that under normal environmental conditions, the first mode of the Alamosa Canyon Bridge varied approximately $5 \%$ over a 24 -hour period, which may be greater than that induced 
by damage and thus may lead to false detection of damage. Alampalli (1998) introduced damage in the bottom flanges of the girders of a bridge used for the test and found the change in natural frequency was smaller than that caused by the freezing of the bridge supports. Kim et al. (2001) studied effect of traffic load on the dynamic characteristics of three bridges and reported a change of up to $5.4 \%$ in the measured natural frequencies was observed with the shortest span steel bridge in the study. It is therefore recognized that the variabilities of operational and environmental effects, induce noise and uncertainties in the captured vibration response, that may either mask the changes caused by structural damages or give a false indication of damage. Thus, in practical applications particularly of structures in the field, the damage detection results of VBSHM are often found to be unreliable. Therefore, in the development of a practical structural health monitoring solution, it is important to distinguish the effects of damage from those caused by variations or uncertainties in the operational and environmental conditions when detecting changes in the dynamic characteristics of the monitored structure.

\subsubsection{Normalization of Field Variables}

In the literature, researchers have developed several statistical methods that claim to account for the effects of operational and environmental variables from the field captured data before being used for damage detection. Although insights on the influence of noise and uncertainties on VBSHM have been gained, most of these previous studies use simulated damage data that lack realistic representation of the actual uncertainties of structures operating in the field. Others are based on experimental tests under highly controlled laboratory testing environment that is altogether different from the noisy and uncertain environment of structures in the field. 
Some examples of previous studies on operational and environmental uncertainties of VBSHM are reviewed herein. Ruotolo and Surface (1997) used singular value decomposition method to determine the rank of matrix consisting of feature vectors (modes/mode shapes/frequency response functions etc.) under different operating conditions in their study of the effect of noise. The method was applied to a laboratory experimental study of 2D trusses. Sohn et al. (2003) used a unique combination of time series forecasting, auto associated neutral networking (ANN) and statistical methods to detect damages under field operating conditions. In the proposed methodology, first a time series forecasting model using auto regressive and auto regressive with exogenous outputs (AR-ARX) is fit into the measured vibration data, obtained under normal operating conditions. Then the extracted coefficients of AR-ARX model obtained from various operational and environmental effects are fed to ANN to characterize the dependency of extracted coefficients on the intrinsic variables (noise and uncertainties). Once the network is properly trained the extracted coefficients used as inputs are reproduced as output with their relationship with field uncertainties. Finally, for any time history signal recorded from an unknown state, the extracted coefficients are fed to the trained ANN to obtain its relationship with field variables. If the prediction error is high, then it shows damage in the structure. The methodology was experimentally tested on an 8 degree-of-freedom model with variation in excitation levels used as variable replicating operational and environmental conditions. Kullaa (2003) proposed an iterative procedure based on factor analysis to eliminate the operational and environmental effects without the need to quantify these effects. The operational and environmental effects were replicated by changing temperature. The method was tested using simulated data and applied to a laboratory 
experiment. Kullaa (2004) attempted to establish nonlinear relationship between the damage sensitive feature and operational and environmental effects and detect the damage using factor analysis. The approach was tested on a finite element model of a vehicle crane. Operational variation was simulated by changing the configuration of the crane vehicle, and damage was simulated by reducing stiffness. Yan et al. (2005a) used principal component analysis and novelty detection statistical technique to detect damages under field operating conditions assuming linear relationship between the structural response parameters and operational conditions. The proposed methodology is tested on a finite element model of a 3-span bridge subjected to load action from temperature gradient. In addition, the methodology is also applied to the testing of a wooden bridge excited by a dynamic shaker. Peeters (2000) attempted to quantify the variabilities of the field data from the Z24 Bridge in Switzerland. Forty-nine sensors installed on the bridge captured the responses and various environmental parameters (temperature, wind characteristics, humidity, etc.) of the bridge over an approximate one-year period prior to its demolition. A relationship between the modal frequencies extracted from the recorded data of the structure and temperature of the environment was established using an autoregression model with exogenous output (ARX). After collecting data for one year, the bridge was artificially damaged. Response data of the damaged structure were again collected. Since the damaged response data were collected under controlled condition, the recorded data do not include the effect of uncertainties from the representative operational and environmental conditions of the structure. The measured frequencies of the damaged structure were compared against the statistical model of the pre-damaged structure which showed significant deviations and the researchers claimed the discrepancies were due to 
damage alone. Yan et al. (2005b) proposed methodology based on principal component analysis (PCA) and novelty statistical detection techniques to identify nonlinear effects of operational influences on structural response parameters. The procedure was validated on the data collected by Peeters (2000). Figueiredo et al. (2010) used a unique combination of associated neutral network, factor analysis, Mahalanobis distance, and singular value decomposition to detect damages in presence of operational and environmental variations. The procedure is tested on a 3 storey frame structure where the changes in stiffness and mass properties were assumed to represent the variations in operational and environmental conditions. Kullaa (2014) proposed gaussian mixture model (GMM) to compensate the nonlinear effects of operational and environmental variables. Then the principal component analysis (PCA) is used to detect the damage. The procedure was validated on the data collected by Peeters (2000), which again did not specifically account for the effects of uncertainties.

The overall observation on the review of previous studies is that the influences of noise and uncertainties have not been adequately considered in the development and testing of the condition assessment and damage detection methods of VBSHM. This is mainly because the opportunities for collecting actual damage data of real structures are rare. Even if there is such an opportunity, the condition of the scenario is likely to be controlled for safety reason and thus is not representative of the variable environment with actual operational and environmental uncertainties in the field. 


\subsection{Objectives}

Recognizing the significant influences of operational and environmental uncertainties on the dynamic characteristics and vibration response of a structure, it is important to account for the effects of noise and uncertainties in the development of methods and procedures of VBSHM. To test the accuracy and effectiveness of existing condition assessment and damage detection algorithms or to develop more effective new methods for realistic VBSHM applications, it is necessary to have an accurate model or procedure that can duplicate the effects of realistic noise and uncertainties from typical operational and environmental conditions of structures in the field. A robust methodology that can simulate damage responses of monitored structures with realistic operational and environmental uncertainties is needed. For a monitored structure with installed sensors to capture vibration response in the field, it is important to recognize that every dataset collected from the monitoring sensors contains the actual and unique operational and environmental uncertainties as occurred at the time when the dataset is collected. Consequently, simulated damage response modified from the original captured undamaged response should exhibit identical operational and environmental uncertainties in both cases and the difference should then be able to be attributed to damage alone.

As such, the objective of the research is to develop a methodology capable of simulating the realistic field damage response of a monitored structure by modifying its captured field response data while preserving all the noise and uncertainty characteristics in the original data. With the developed methodology and procedure for easy generation of damage vibration response data under different damage scenarios, existing condition assessment and damage detection algorithms can be tested and improved on their effectiveness, or new 
assessment algorithms can be developed to solve real world SHM problems under realistic field conditions.

\subsection{Methodology}

\subsubsection{Overview}

The proposed methodology for simulating realistic damage response is a multivariate output only procedure based on modification of actual field captured vibration response sensor data while preserving the embedded field noise and uncertainties already in the data. The procedure is based on the theory of structural dynamics and considering the full noise and uncertainties as included in any ambient vibration response data captured by sensors on monitored structures in the field. In the formulation of the proposed method, a new concept of Pseudo load is introduced which can be used to represent the load actions corresponding to the captured vibration response of the monitored structure. For typical civil engineering structures operating under ambient conditions in the field, the load actions which cause the captured vibration response by the monitoring system are generally not known. However, by using a finite element model of the monitored structure, these previously unknown load actions can be quantified by derivation of the Pseudo load of the captured response dataset. In the simulation of the damage vibration response, the derived Pseudo load is then applied to a modified computer model of the structure that corresponds to the simulated damaged state of the structure to generate the damage vibration response. The proposed new method can easily be applied to simulate the vibration response of the 
structure under any damage scenario with all the noise and uncertainty effects from the field variables preserved in their original form, as shown in Fig. 2.1.

\subsubsection{Derivation of Pseudo Load}

One of the biggest challenges in simulating a realistic damage response of a structure under field operational and environmental conditions is that it is impossible or impractical to measure or quantify all the ambient variables, whether related to the load actions or the environment for a structure operating in the field. In other words, only the output from the ambient operational load and environmental actions in the form of field measured responses are known, but not the forces causing those responses. Alternatively, if the field recorded vibration response includes the full set of acceleration $\ddot{u}_{n}$, velocity $\dot{u}_{n}$ and displacement $u_{n}$ time history responses of a structure, the full time history of the dynamic load actions corresponding to the recorded dynamic response can easily be derived from the equation of motion of the structure, as shown in Eq. 2.1.

$$
[\mathrm{M}] \ddot{u}_{n}+[\mathrm{C}] \dot{u}_{n}+[\mathrm{K}] u_{n}=F_{n}
$$

where $[\mathrm{M}],[\mathrm{C}]$, and $[\mathrm{K}]$ are the mass, damping and stiffness matrix, respectively; $\ddot{u}_{n}$, $\dot{u}_{n}$ and $u_{n}$ denote the field recorded acceleration, velocity and displacement response at $\mathrm{n}$ measuring locations of the structure; $F_{n}$ is the dynamic load on the monitored structure. As the left-hand side of the equation of motion shown in Eq. 2.1 is completely deterministic and equal to the load actions on the right-hand side of the equation, this load action time history can then be used to generate damage response of any assumed damage scenarios of the structure, as shown in Fig. 2.2. The calculated load, if used as input excitation in the finite element model of the monitored structure, as shown in Eq. 2.1, will produce the same 
captured responses $\left(\ddot{u}_{n}, \dot{u}_{n}\right.$ and $\left.u_{n}\right)$. Therefore, all the noise and uncertainties present in the captured response are preserved in the calculated load $F_{n}$ since the load $F_{n}$ includes all the inherent field uncertainties, the application of the same dynamic load to the simulated damaged structure will result in the simulated damage vibration response having the same uncertainty effects as in the original response. With the captured responses $\left(\ddot{u}_{n}, \dot{u}_{n}, u_{n}\right)$ and the simulated damage responses $\left(\ddot{u}_{n d}, \dot{u}_{n d}, u_{n d}\right)$ having the same identical field uncertainties, any difference in the dynamic characteristics between the two structural states will be due to damage alone. Having a method capable to generate any damage vibration response while preserving the full characteristics of naturally embedded ambient noise and uncertainties in field captured vibration response data, the simulation results can be used to develop more practical VBSHM tools for real world applications in the field.

In typical vibration measurement systems of structural health monitoring applications, it is common that acceleration responses are measured by accelerometers and not velocity and displacement. As a result, it is not possible to use the aforementioned procedure for determining the dynamic load time history $F_{n}$, as acceleration is the only captured vibration response for a monitored structure. Therefore, the velocity and displacement responses can only be obtained by numerical integration of the field measured acceleration responses of the structures. However, the initial conditions of the structure as required by numerical integration are not known. To overcome this lack of information on the initial conditions of the structure, the concept of Pseudo load is introduced. By means of numerical integration of the captured acceleration response, velocity and displacement time history responses can be calculated by assuming zero initial conditions. As shown in Eqs. 2.2 and 
2.3, integrating the measured acceleration $\left(\ddot{u}_{n}\right)$ response twice to get velocity and displacement response

$$
\begin{aligned}
& \dot{u}_{n}=\int \ddot{u}_{n} d t+V_{n} \\
& u_{n}=\iint \ddot{u}_{n} d t+V_{n} t+S_{n}
\end{aligned}
$$

where $V_{n}$ and $S_{n}$ are the unknown initial conditions of initial velocity and initial displacement respectively at the corresponding $n$ sensor measurement locations. By rearranging, the above integrals can be rewritten as follows

$$
\begin{aligned}
& \dot{\tilde{u}}_{n}=\int \ddot{u}_{n} d t=V_{n} \\
& \tilde{u}_{n}=\iint \ddot{u}_{n} d t=-S_{n}
\end{aligned}
$$

Substituting Eqs. 2.4 and 2.5 into Eq. 2.1 gives

$$
[\mathrm{M}] \ddot{u}_{n}+[\mathrm{C}] \dot{\tilde{u}}_{n}+[\mathrm{K}] \tilde{u}_{n}=F_{n}-[\mathrm{C}]\left\{V_{n}\right\}-[\mathrm{K}]\left\{V_{n} t+S_{n}\right\}=P_{\text {pseudo }}
$$

The right-hand side of Eq. 2.6 is the definition of the Pseudo load in geometric coordinates given by the left-hand side of Eq. 2.6 in terms of the field measured acceleration response $\ddot{u}_{n}$ and the calculated field displacement $\tilde{u}_{n}$ and velocity response $\dot{\tilde{u}}_{n}$ with zero initial conditions. The difference between the exact dynamic load and the Pseudo load is the discrepancy of not considering the free vibration response from initial conditions of the structure. Except for the discrepancy of the initial conditions, it is important to recognize that the Pseudo load contains all the effects of noise and uncertainties from the operational and environmental conditions of the structure as embedded in the captured acceleration response of the structure. By applying the derived Pseudo load to any damage state of the 
structure, the simulated damage vibration response of the damaged structure can be generated. The simulated damage response is exact with completely preserved characteristics of the noise and uncertainties as embedded in the original captured acceleration vibration response signal with the exception of ignoring the effects of the initial conditions of the structure. The concept of the Pseudo load and its application in simulations of damage vibration response are shown in Fig. 2.3.

For typical civil engineering structures such as buildings and bridges, the effect of initial condition on the dynamic response of a structure quickly diminishes due to damping or energy dissipation properties of the structure. Therefore, after the dissipation of the free vibration response of the structure due to initial conditions, the simulated damage vibration response derived by the application of Pseudo load to the structure is the same as that derived with the exact load and initial conditions. The simulated damage response of a monitored structure computed using the exact load and the one using the Pseudo load are not the same because Pseudo load as given in Eq. 2.6 assumes zero initial conditions. However, it is shown in the next section that this lack of consideration of initial conditions does not affect the validity of the simulated damage acceleration response as the rest of the simulated damage acceleration response using the exact loads $\ddot{u}_{n d}$ and that using Pseudo load $\ddot{u}_{n p d}$ are the same after the free vibration response resulting from the initial conditions of the structure dies out. This observation is demonstrated in numerical examples presented in Section 2.5. 


\subsubsection{Response due to Initial Conditions}

The validity of using the Pseudo loads calculated from Eq. 2.6, as input excitation to simulate the field acceleration responses in the damage state is a concern since the Pseudo load lacks the information of the initial conditions in the forcing functions. In this section, it is shown that the response of a structure due to initial conditions decays quickly. Thereafter, the simulated acceleration response of the damaged structure using Pseudo load becomes the same as the exact damage response of the structure.

Considering the acceleration $\ddot{u}_{i}$, velocity $\dot{u}_{i}$ and displacement response $u_{i}$ of a single degree-of-freedom (sdof) system with $M, C$, and $K$ as the mass, damping and stiffness respectively due to arbitrary initial conditions. The equation of motion of the sdof system from Eq. 2.6 is given by Eq. 2.7.

$$
\begin{aligned}
& M \ddot{u}_{i}+C \dot{u}_{i}+K u_{i}=-C\left(\mathrm{~V}_{O}\right)-K\left(\mathrm{~V}_{O} \mathrm{t}+\mathrm{S}_{O}\right) \text { or } \\
& M \ddot{u}_{i}+C \dot{u}_{i}+K u_{i}=-C \mathrm{~V}_{O}-K \mathrm{~S}_{O}-K \mathrm{~V}_{O} \mathrm{t} \text { or } \\
& \ddot{u}_{i}+\mathrm{c} \dot{u}_{i}+\mathrm{k} u_{i}=\mathrm{At}+\mathrm{B}
\end{aligned}
$$

where $\mathrm{S}_{O}$ is the initial displacement, and $\mathrm{V}_{O}$ is the initial velocity, $\mathrm{A}=-K\left(\mathrm{~V}_{O} \mathrm{t}\right) / M$, $\mathrm{B}=-K\left(\mathrm{~V}_{O} \mathrm{t}+\mathrm{S}_{O}\right) / M, \mathrm{c}=C / \mathrm{m}$ and $\mathrm{k}=K / M$,

where right-hand side of Eq. 2.9 are forcing function due to the initial conditions $\left(\mathrm{S}_{O}\right.$ and $\mathrm{V}_{O}$ ). Multiplying the differential operator $D^{2}$ on both sides of Eq. 2.9, where $\mathrm{D}=\frac{d}{d t}$, gives

$$
D^{2}\left(D^{2}+\mathrm{Dc}+\mathrm{k}\right) u_{i}=0
$$

Solving Eq. 2.9 gives the roots of 


$$
D= \pm 0 \text { and } D=\frac{-c \pm \sqrt{c^{2}-4 k}}{2}
$$

For an underdamped system,

$$
0.5 \sqrt{\mathrm{c}^{2}-4 \mathrm{k}}=\lambda<1
$$

Resulting in the complex roots

$$
D=\frac{-c \pm i \sqrt{4 k-c^{2}}}{2}
$$

Therefore, the general solution of Eq. 2.10 with four roots gives

$$
u_{i}=\left(H_{1} t+H_{2} B\right)+e^{\frac{-c t}{2}}\left(G_{1} \sin \lambda t+G_{2} \cos \lambda t\right)
$$

where $H_{1}, H_{2}, G_{1}$ and $G_{2}$ are the unknown constants obtained from the unknown initial conditions. The acceleration response after differentiating Eq. 2.14 twice gives

$$
\begin{aligned}
\ddot{u}_{i}= & e^{\frac{-c t}{2}}\left[-G_{1} \lambda^{2} \sin \lambda t-G_{2} \lambda^{2} \cos \lambda t\right]-\frac{c}{2} e^{\frac{-c t}{2}}\left[G_{1} \lambda \sin \lambda t-G_{2} \lambda \cos \lambda t\right]- \\
& \frac{c}{2} e^{\frac{-c t}{2}}\left[G_{1} \lambda \cos \lambda t-G_{2} \lambda \sin \lambda t\right]+\frac{c^{2}}{4} e^{\frac{-c t}{2}}\left[G_{1} \sin \lambda t+G_{2} \cos \lambda t\right]
\end{aligned}
$$

The terms on the right-hand side of Eq. 2.15 are subjected to exponential decay. Therefore, given long enough duration, the response due to the initial conditions will eventually die out and the simulated acceleration response by the Pseudo load will become the same as the exact response of the structure. 


\subsection{Numerical Examples}

\subsubsection{Ambient Scenarios}

Ambient vibration scenarios are characterized by long duration continuous vibrations with non-zero initial conditions of amplitude likely to be of the same order of the peak response. Highway bridges and buildings in urban areas are subjected to ambient loads, such as wind loads and traffic loads on the structure, as in the case of bridges, or nearby traffic for buildings, causing vibration response of the structures. In the following numerical example, a building in a typical urban area is subjected to ambient load actions represented by random loads. The model of a 4 -storey single bay moment resisting frame building is shown in Fig. 2.4. The properties of the structure are provided in Table 2.1. A sensor is assumed on each floor that captures the acceleration response in the lateral direction.

For the example building, as shown in Fig 2.4, the mass and stiffness matrices of the building are assumed as follows

$[\mathrm{M}]=\left[\begin{array}{cccc}10 & 0 & 0 & 0 \\ 0 & 10 & 0 & 0 \\ 0 & 0 & 10 & 0 \\ 0 & 0 & 0 & 10\end{array}\right] \mathrm{kNsec}^{2} / \mathrm{m}, \quad[\mathrm{K}]=\left[\begin{array}{cccc}200 & -100 & 0 & 0 \\ -100 & 200 & -100 & 0 \\ 0 & -100 & 200 & -100 \\ 0 & 0 & -100 & 100\end{array}\right] \mathrm{kN} / \mathrm{m}$

The building is assumed to have 5\% Rayleigh damping in first two vibration modes.

In the absence of actual field recorded acceleration data, four randomly generated time history signals, as shown in Fig. 2.5, at a sampling frequency of $50 \mathrm{~Hz}$ are used as input loads. The corresponding lateral acceleration responses of the building with zero initial conditions are shown in Fig. 2.6. To account for the effect from the initial conditions, the displacement and velocity responses at $\mathrm{t}=20$ seconds are taken as the initial conditions, as 
shown in Table 2.2. For the simulation of the damage response, the base column is assumed to have damage of reduced lateral stiffness by $10 \%$ while those of the remaining columns are reduced by $5 \%$. The mass matrix is assumed to remain the same while the stiffness matrix of the damaged structure changes accordingly.

$\left[\mathrm{M}_{\mathrm{d}}\right]=\left[\begin{array}{cccc}10 & 0 & 0 & 0 \\ 10 & 10 & 0 & 0 \\ 0 & 0 & 10 & 0 \\ 0 & 0 & 0 & 10\end{array}\right] \mathrm{kNsec}^{2} / \mathrm{m} \quad\left[\mathrm{K}_{\mathrm{d}}\right]=\left[\begin{array}{cccc}185 & -95 & 0 & 0 \\ -95 & 190 & -95 & 0 \\ 0 & -95 & 190 & -95 \\ 0 & 0 & -95 & 95\end{array}\right] \mathrm{kN} / \mathrm{m}$

To generate the Pseudo loads given in Eq. 2.6, the acceleration responses from $t=20 \mathrm{sec}$ onwards are integrated to obtain the velocity and displacement response with zero initial conditions, the Pseudo loads are then applied to the damaged structure to obtain the simulated acceleration vibration response. The simulated damage responses by the Pseudo load method are compared with the exact damage response of the structure.

Figure 2.7 shows the percentage normalized error in the damage acceleration response obtained from Pseudo loads with respect to exact damage response at degrees-of-freedom 2 (mid height of example building) and 4 (roof of the example building). The error in the simulated damage results obtained from Pseudo loads from the exact results obtained from the exact loads that include initial conditions, can be observed during the first 20 seconds. The magnitude of error in the results is expected to be high initially due the initial conditions being relatively high compared to the peak responses of the structure under ambient load conditions. The discrepancies due to the initial conditions can be seen decaying quickly as predicted by Eq. 2.15 and has very little influence beyond 20 seconds of the response. 


\subsubsection{Discrete Events}

The simulation of damage vibration response of a structure subjected to the load effects of an extreme discrete event, such as an earthquake, is investigated herein. The recorded initial vibration response of a structure subjected to the load actions of a major earthquake is typically relative calm at the beginning. This indicates that the effect of initial conditions is small in comparison to the peak responses during the strong shaking of the earthquake excitation. Consequently, it is expected that the initial conditions would have minimal influence on the simulated damage acceleration response of the structure using the proposed Pseudo load method. To demonstrate the accuracy of the Pseudo load method for simulating damage response to extreme discrete load events such as earthquakes, the recorded earthquake acceleration response data captured by accelerometers on a 3-story building in Richmond, California (CESMD), as shown in Fig. 2.8, are used in the illustration. The recorded acceleration response data recorded at a sampling rate of $100 \mathrm{~Hz}$ from a magnitude 4.4 MW earthquake in Berkeley on January 4, 2018, as shown in Fig. 2.9 , are used in the investigation here.

For convenience, a 3 degrees-of-freedom cantilever model of the building is adopted and each dof has a sensor that continuously records the acceleration response in the lateral direction. Although the model adopted herein is not the correct representation of the actual building, this modelling assumption does not affect the validity of the proposed method for simulation of damage vibration response of the building. This is because any discrepancies in the modelling of the geometric and material properties of the building as well as discrepancies in the behaviour in the assumed structural model with respect to the actual building will be carried over to its damaged state. 
Therefore if $\left[\mathrm{K}_{\mathrm{A}}\right]$ is the actual stiffness matrix of the structure while $\left[\mathrm{K}_{\mathrm{CH}}\right]$ is the stiffness matrix of the assumed cantilever model, considering only static response, the actual Pseudo load and Pseudo load from cantilever model can be calculated as follows

$$
\begin{gathered}
{\left[\mathrm{K}_{\mathrm{A}}\right] \tilde{u}_{n}=P_{\text {pseudoA }}} \\
{\left[\mathrm{K}_{\mathrm{CH}}\right] \tilde{u}_{n}=P_{\text {pseudocH }}}
\end{gathered}
$$

Clearly actual Pseudo load $P_{\text {pseudoA }}$ and Pseudo load calculated from cantilever model $P_{\text {pseudoch }}$ are different because of the error in the stiffness of the cantilever model. However, the calculated Pseudo load $P_{\text {pseudocH }}$, when used as input excitation with the cantilever model, generates the captured response $\tilde{u}_{n}$. This shows that $P_{\text {pseudoct }}$ has the identical field noise and uncertainties as present in the captured response $\tilde{u}_{n}$. The simulated damage response $\tilde{u}_{n d}$ obtained by applying the Pseudo load to the damaged building given by the damage stiffness $\left[\mathrm{K}_{\mathrm{CD}}\right]$ using the assumed cantilever model, as shown in Eq. 2.18, is not the actual damage response of the building. This does not affect the validity and accuracy and effectiveness of the proposed pseudo load method. The use of the assumed structural model for the example here is just for illustration purposes.

$$
\left[\mathrm{K}_{\mathrm{CD}}\right] \tilde{u}_{n d}=P_{\text {pseudocH }}
$$

Therefore, the simulated damage response of the cantilever model $\tilde{u}_{n d}$ will have the identical noise and uncertainties as present in the captured response $\tilde{u}_{n}$ and the damage characteristics of the model. Such vibration data can then be used to examine the effectiveness of vibration based damage detection algorithms in identifying damages when the structural response parameters are mixed with noise and uncertainties. 
The assumed mass and stiffness matrices of the assumed cantilever model in healthy state, are obtained from the structural properties given in Table 2.3 are shown as follows

$[\mathrm{M}]=\left[\begin{array}{ccc}10 & 0 & 0 \\ 0 & 10 & 0 \\ 0 & 0 & 10\end{array}\right] \mathrm{kN} \sec ^{2} / \mathrm{m} \quad[\mathrm{K}]=\left[\begin{array}{ccc}600 & -200 & 0 \\ -200 & 400 & -200 \\ 0 & -200 & 200\end{array}\right] \mathrm{kN} / \mathrm{m}$

To account for the effect of initial conditions, the Pseudo load is first calculated using Eq.2.6 and field recorded acceleration response data (CESMD). The Pseudo load is used as input excitation at the 3 dof's of the model to obtain the structural responses. The dynamic responses at $\mathrm{t}=30$ seconds are taken as the initial conditions of the structure as shown in Table 2.4. Then following the same procedure as presented in Section 2.5.1, the simulated damage response can be calculated. For the simulated damage scenario, the base column is assumed to have damage of a reduction to its stiffness by $10 \%$ and that for the columns in the upper storeys by $5 \%$. The mass and stiffness matrices of the damaged state are as follows

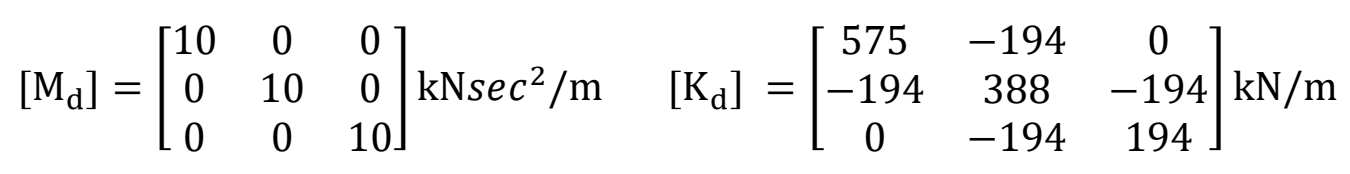

Since the acceleration vibration response data are captured by sensors in the monitored building in the field, it is assumed the captured data include all the field variabilities and uncertainties of the operational and environmental conditions of the structure at the time of the data capture. The normalized error in the simulated damage response obtained by the Pseudo load method in comparison to the exact damage responses are shown in Fig. 2.10. The error is significant during the first 3 to 5 seconds of an overall duration of 42.78 seconds. 


\subsubsection{Phase Preservation}

The responses captured by the sensors of a monitored structure are related to each other through the modal properties of the structure. For each vibration mode, the mode shape of the structure defines the relative amplitude and phase difference between the dof's of the structure. In the simulation of the damage acceleration response, it is important that the phase properties between the sensor dof's of the monitored structure are not altered. Otherwise the modal properties extracted using the simulated acceleration responses will not be correct and do not represent the true vibration behaviour of the damaged structure. In the proposed Pseudo load methodology for damage response simulations, a criterion is established for selecting the response data where the influence of initial conditions has sufficiently dissipated. The criterion is based on Eq. 2.19 which limits the normalized relative error of the simulated damage response with respect to the peak response of the exact result to less than $1 \%$.

$$
\Delta_{n}=\left[\frac{\delta_{n}}{\ddot{u}_{n_{\text {max }}}}\right]<0.01
$$

where $\delta_{n}$ is the absolute difference between the exact damage acceleration response and the simulated damage response by the Pseudo load method for each $n$ data channel; and $\ddot{u}_{n_{\max }}$ represents the maximum exact damage acceleration response of a monitored structure with $n$ data channels.

Figure 2.11 shows the normalized relative error of the simulated damage response at the first and third storey of the discrete event example building. It shows that the simulated damage responses at the first and third storey satisfy the criterion of Eq. 2.19 at $2.36 \mathrm{sec}$ and $0.22 \mathrm{sec}$ respectively out of the total duration of $42.97 \mathrm{sec}$ of the earthquake. The exact 
and simulated damage response by the Pseudo load method are shown in Figs. 2.12 and 2.13. After applying the criterion given in Eq. 2.19, the phase relationships of the exact and simulated damage responses by the Pseudo load method at first floor is shown in are shown in 2.14. Since the phase relationship between exact response and response simulated by pseudo loads are same therefore the simulated damage response has identical noise and uncertainties as present in captured response and the damage characteristics of the cantilever model.

Figure 2.15 shows the normalized relative error of the simulated damage response at the mid height and roof of the ambient scenario example building. It shows that the simulated damage responses at the mid height and roof satisfy the criterion of Eq. 2.19 at $15 \mathrm{sec}$ and $19.5 \mathrm{sec}$ respectively out of the total duration of $180 \mathrm{sec}$ of the structural response. The exact and simulated damage response by the Pseudo load method are shown in Figs. 2.16 and 2.17. After applying the criterion given in Eq. 2.19, the phase relationships of the exact and simulated damage responses by the Pseudo load method at the roof is shown in Fig. 2.18. Since the phase relationship between exact response and response simulated by pseudo loads are same therefore the simulated damage response has identical noise and uncertainties as present in exact damage response. 


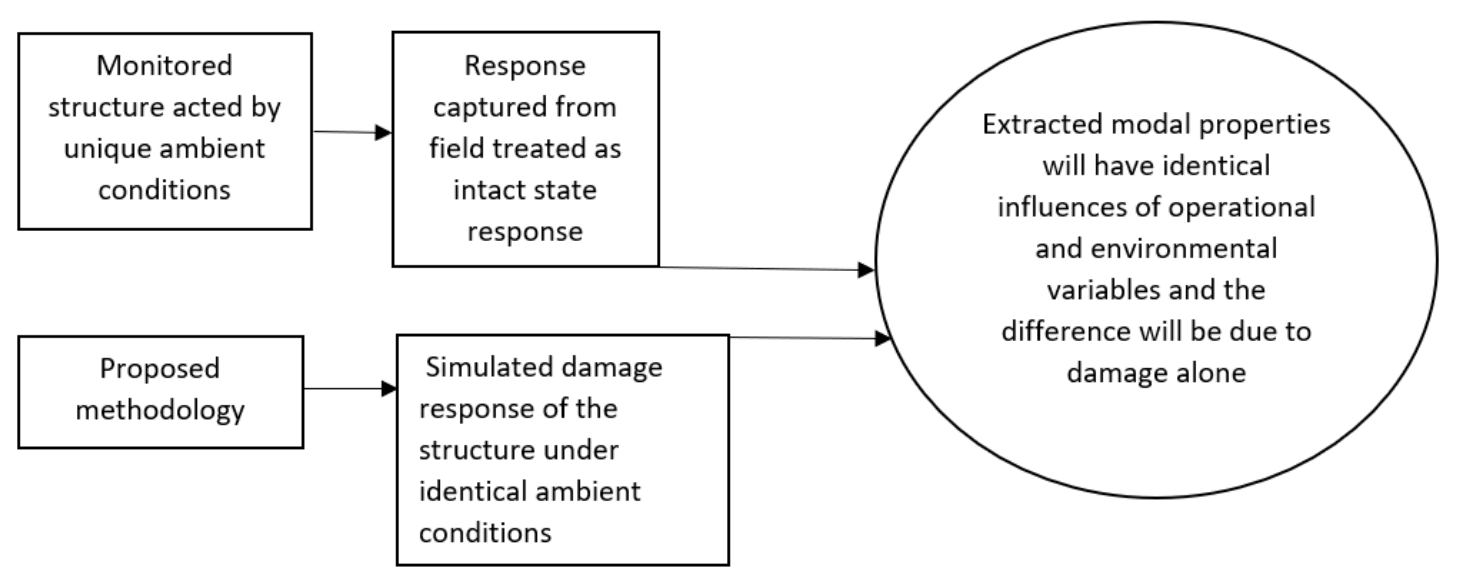

Figure 2.1. Basic focus of the research. 


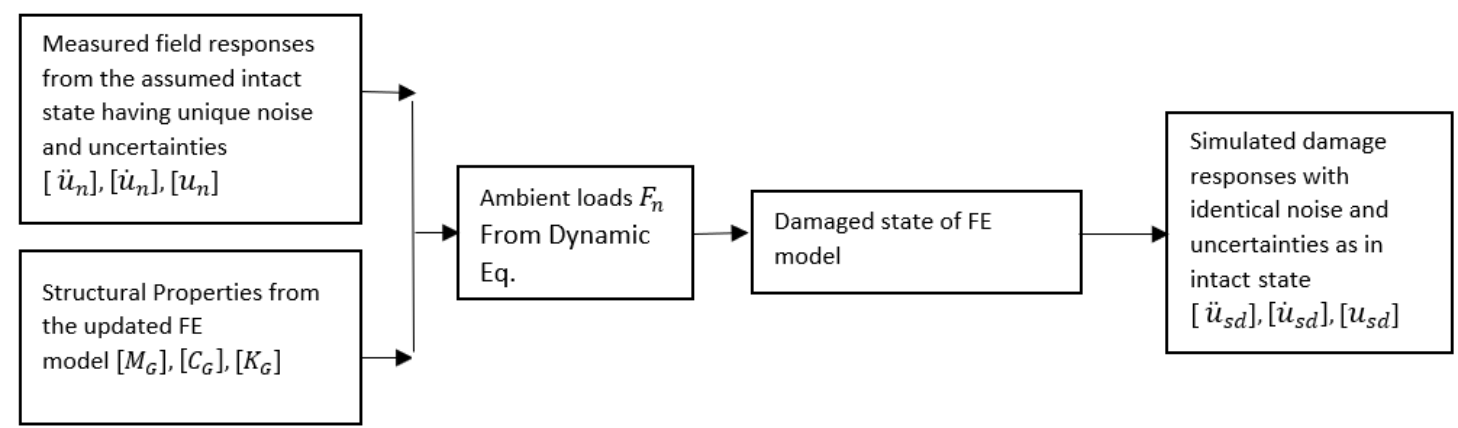

Figure 2.2. The schematic diagram of how damage responses can be simulated from measured responses and finite element model. 
a)

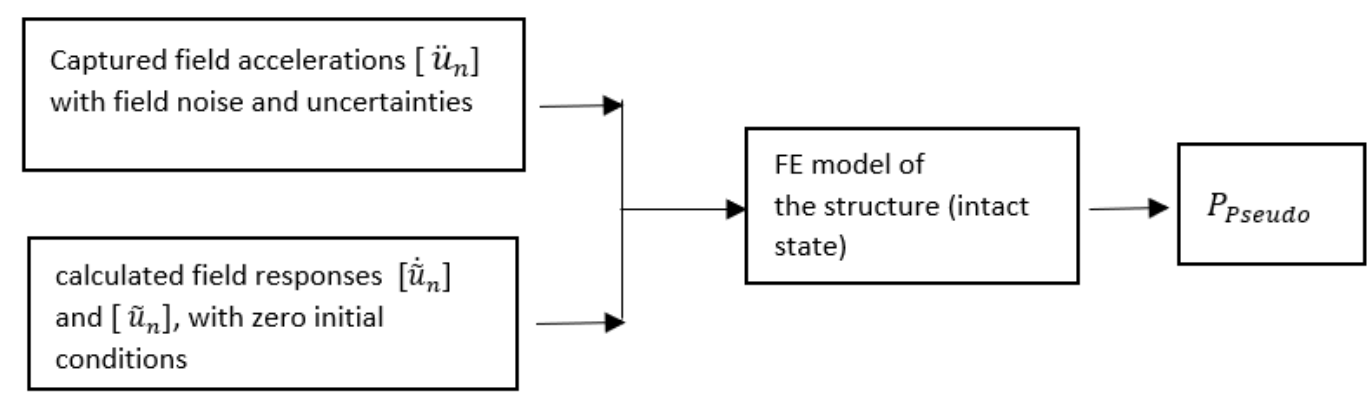

b)

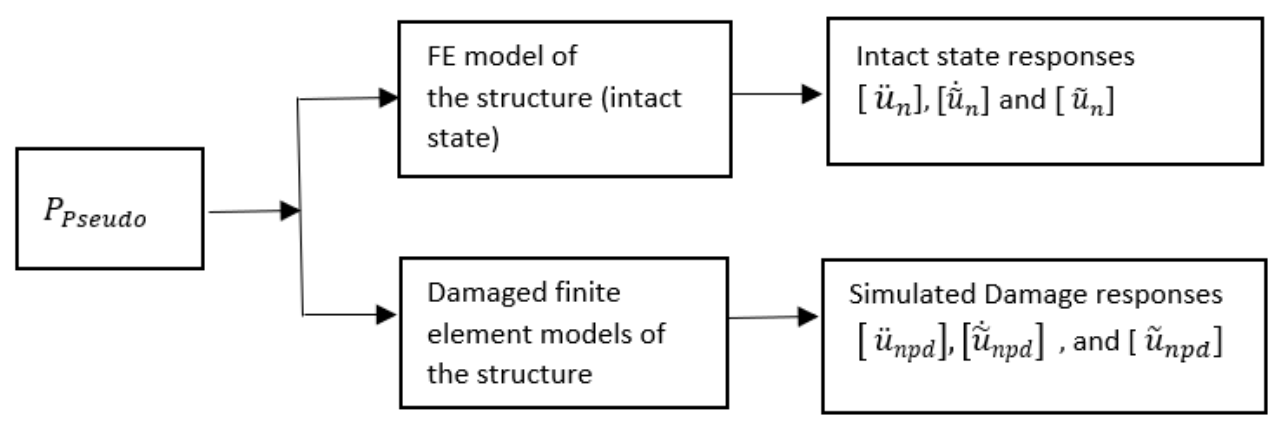

Figure 2.3. (a) How to compute Pseudo loads and (b) how Pseudo loads can be used to simulate damage responses. 


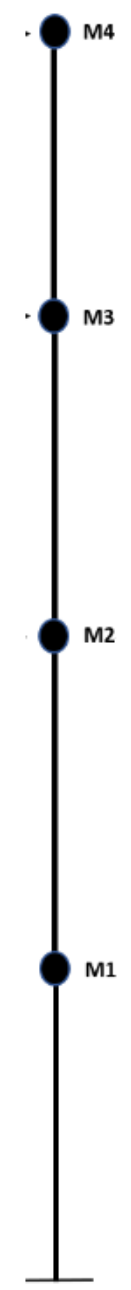

Figure 2.4. Structural model of the four storey moment resisting frame building example. 


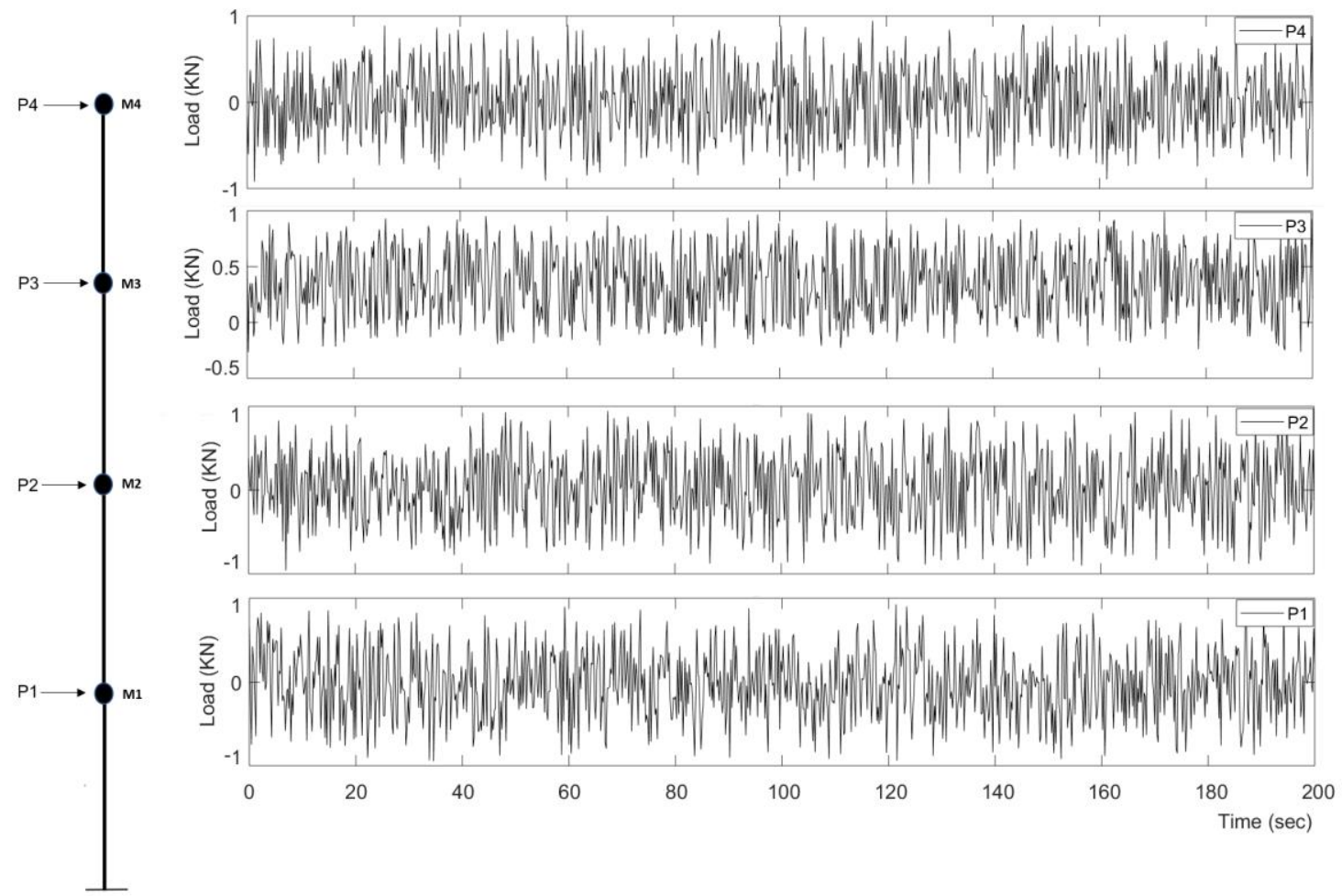

Figure 2.5. Random loads for the example building. 


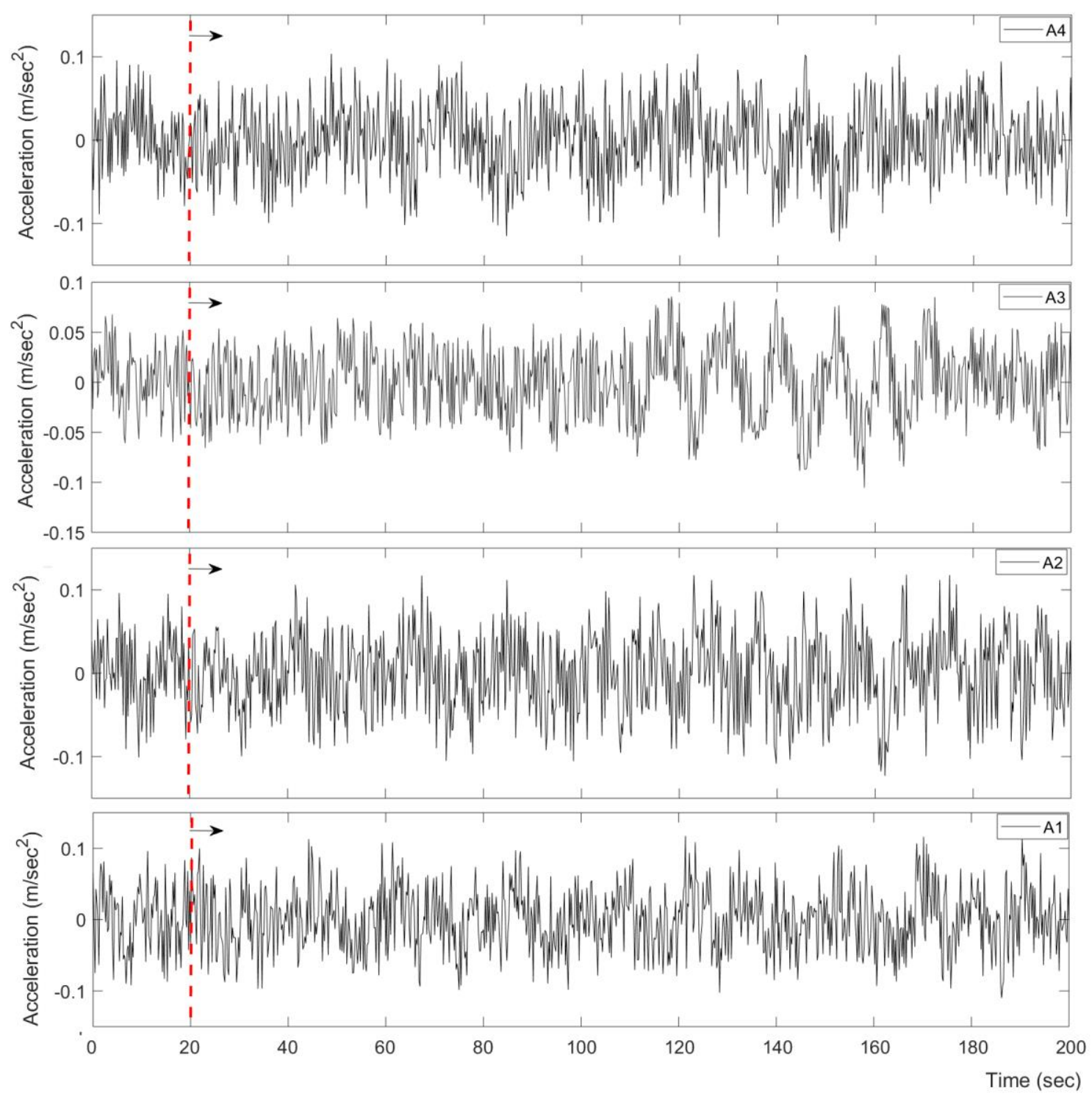

Figure 2.6. Acceleration responses of the example building. 

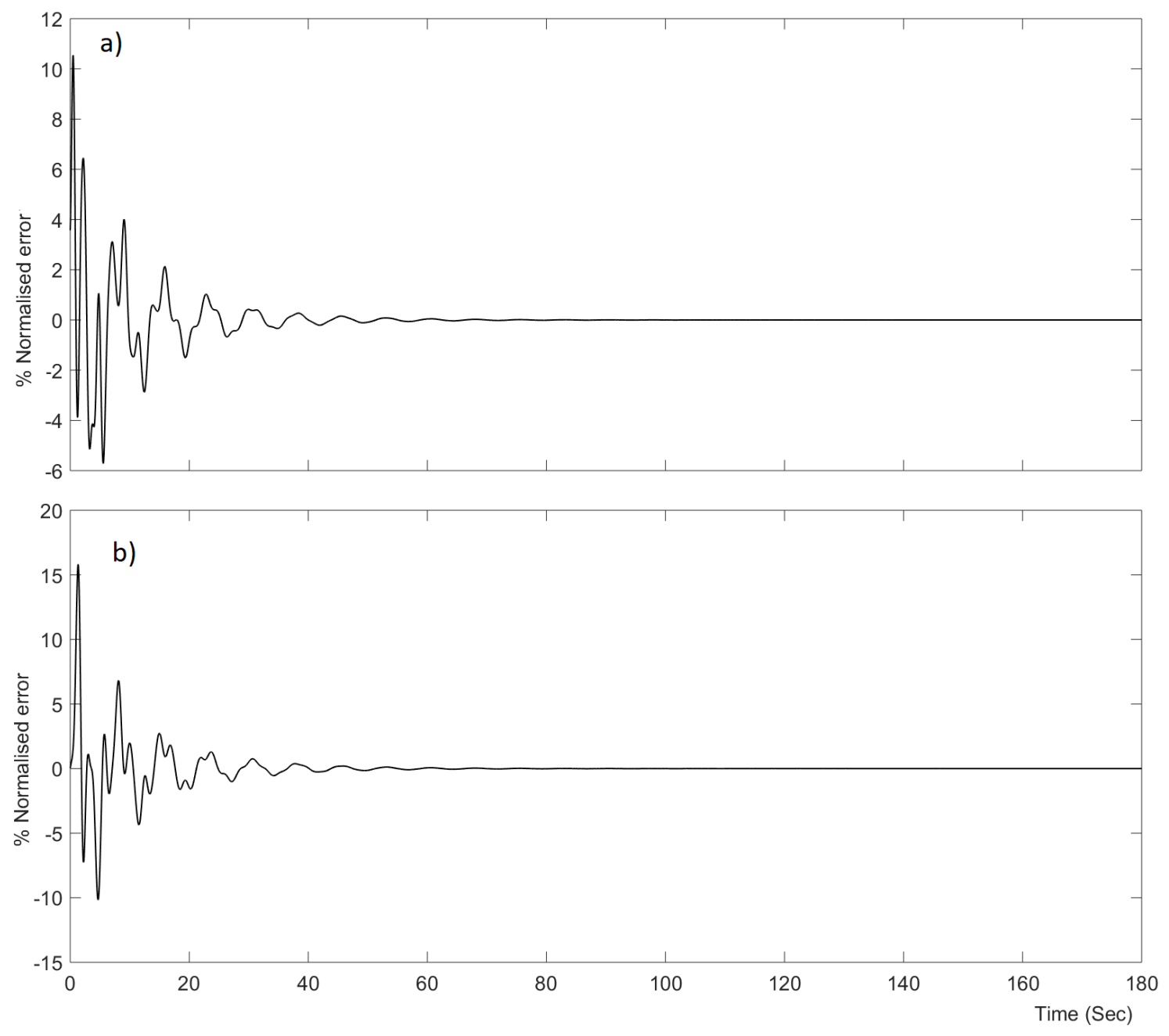

Figure 2.7. Percentage normalized error in the damage response simulated by Pseudo load at; (a) mid-height (dof 2) and (b) roof (dof 4). 
a)

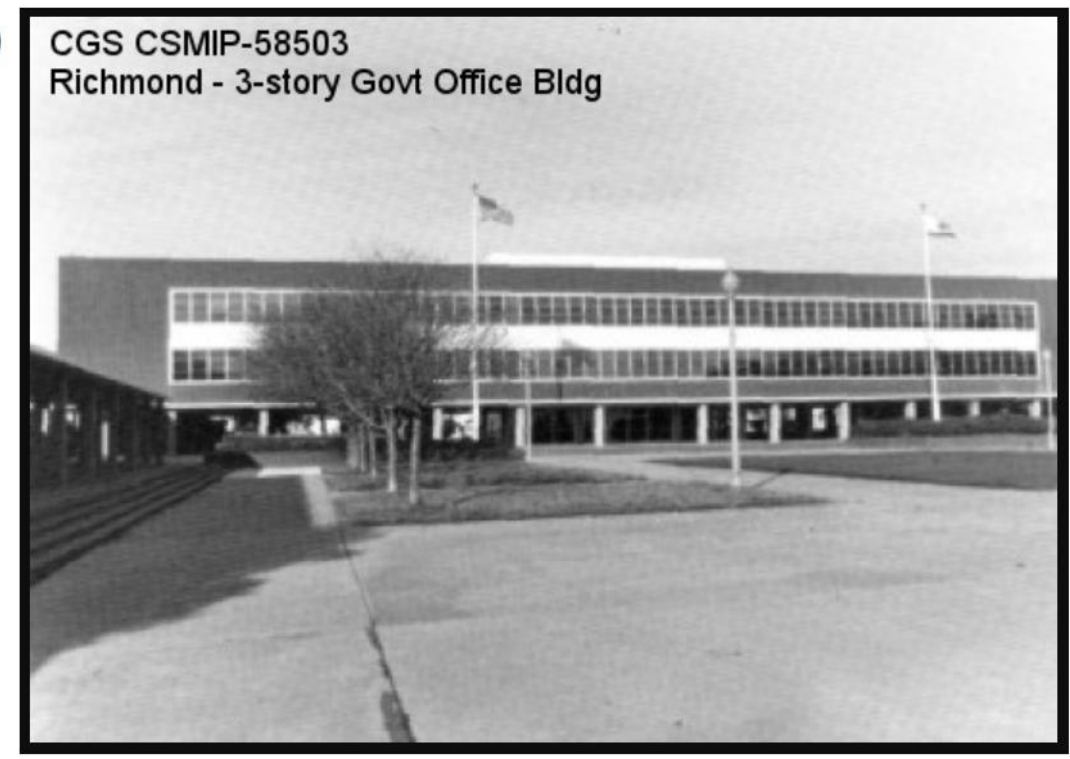

b)

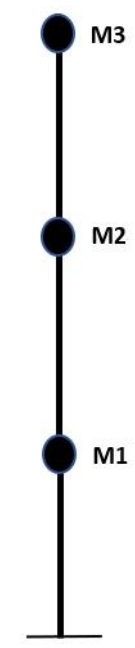

Figure 2.8. Picture of the building in Richmond, California with accelerometers installed at all the floor levels, provided by The Centre of Engineering Strong Motion Data (CESMD) in website www.strongmotioncentre.org (b) simplified model adopted for simulation. 

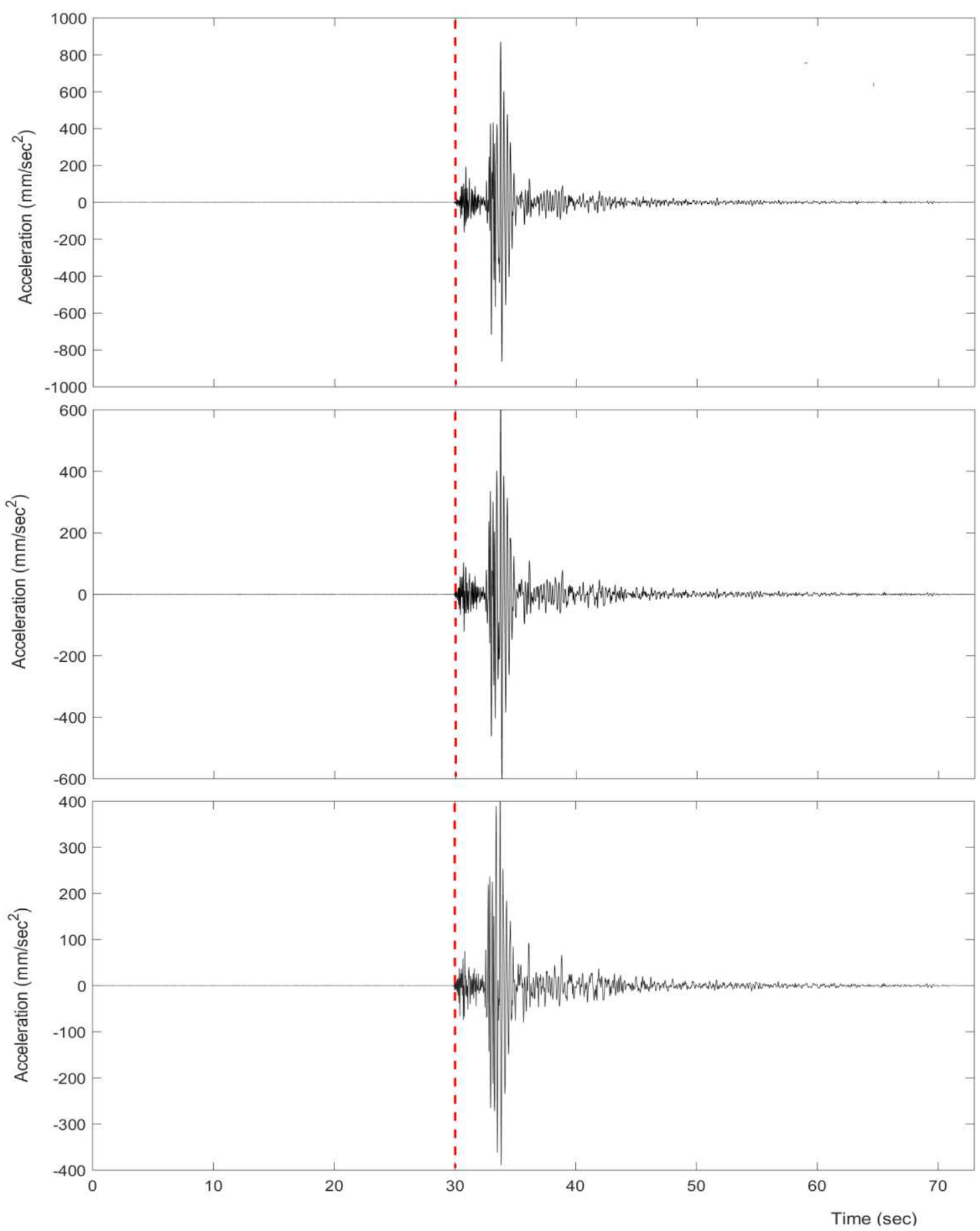

Figure 2.9. Acceleration response recorded by the sensors at the three floor levels in EW lateral direction of the building provided by The Centre of Engineering Strong Motion Data (CESMD) in website www.strongmotioncentre.org. 

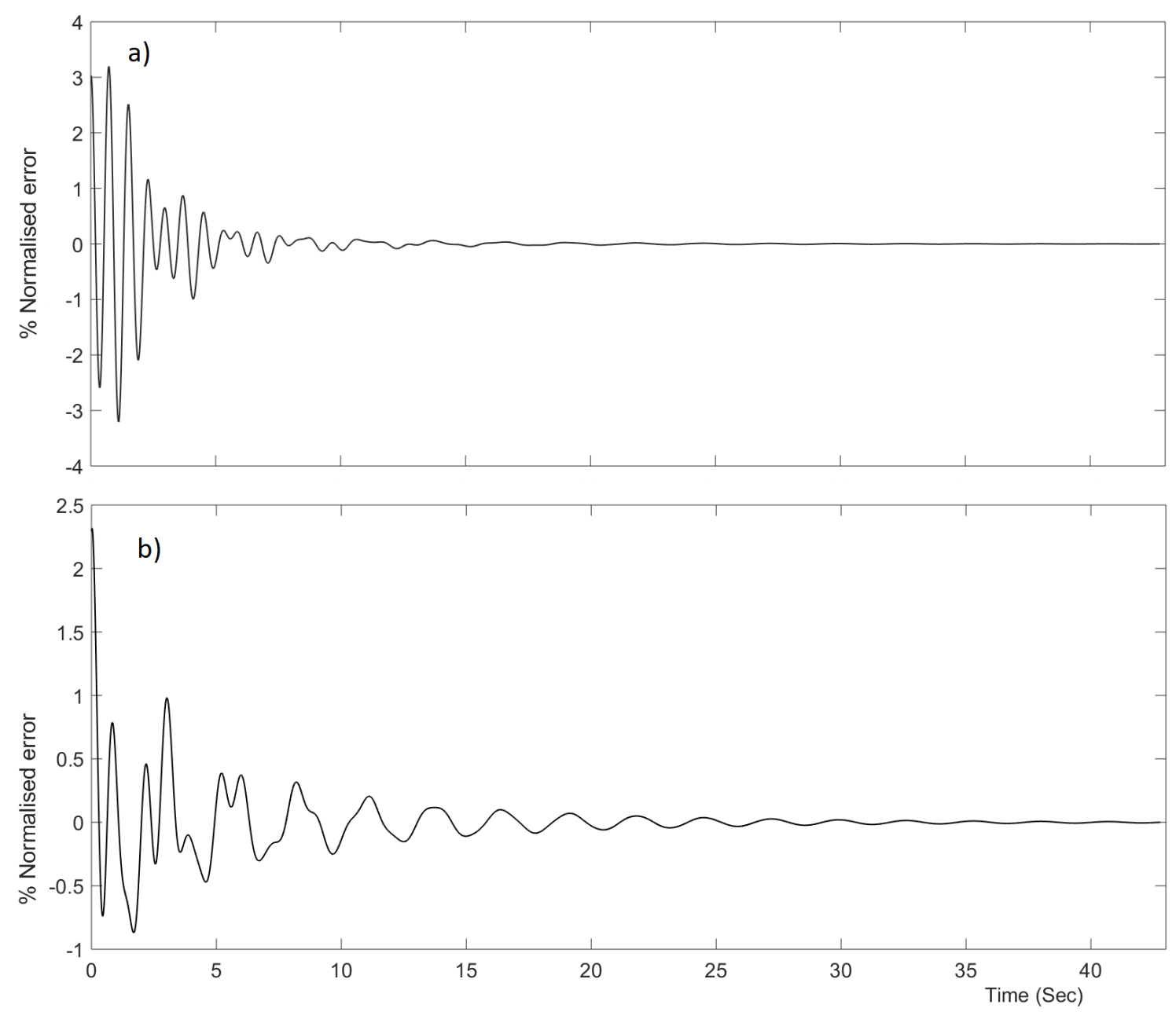

Figure 2.10. Percentage normalized error in the response simulated by Pseudo load at; (a) first floor (dof 1) and (b) roof (dof 3), of the model adopted in discrete event example. 

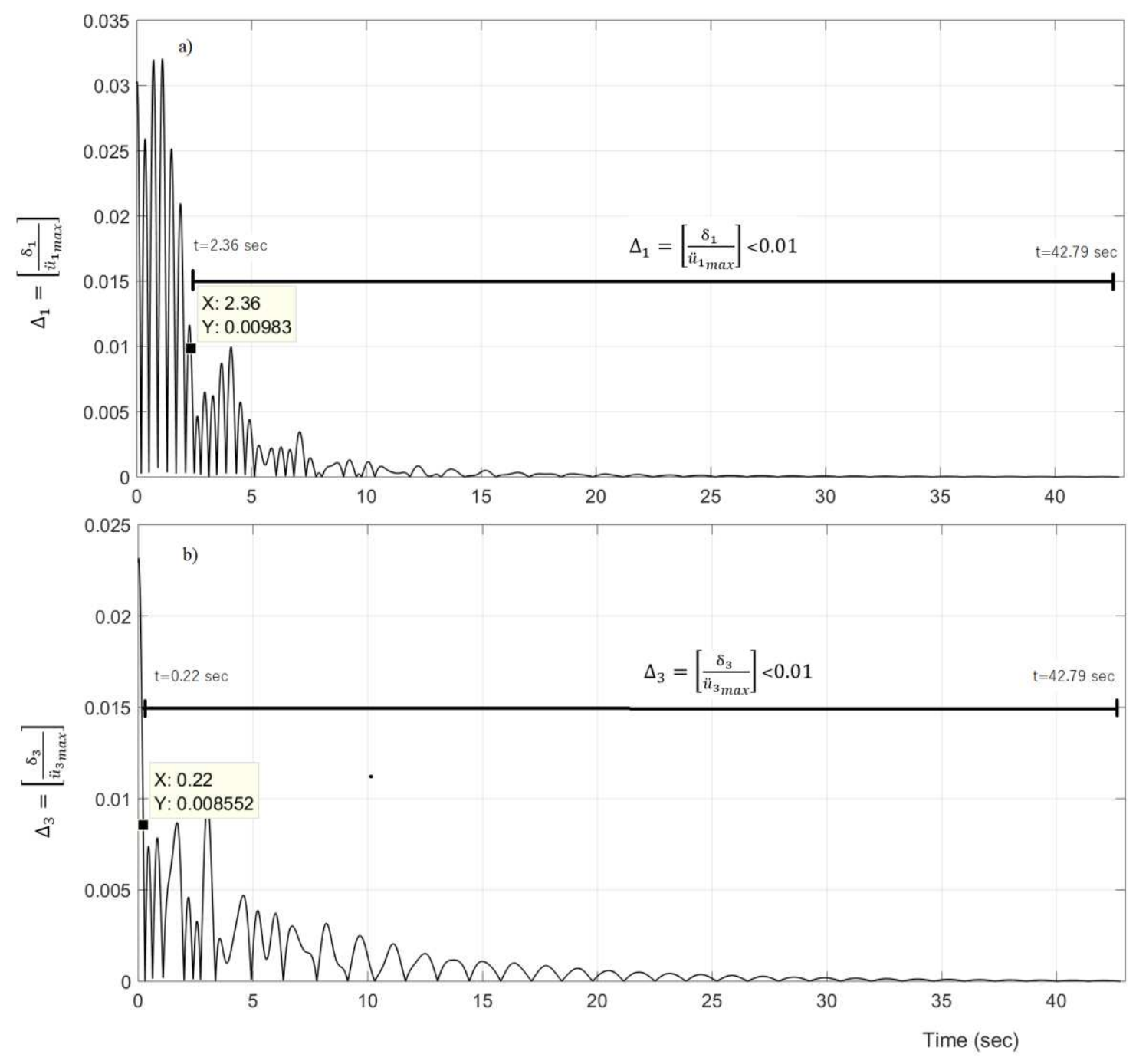

Figure 2.11. Normalized relative error in the response simulated by Pseudo load at;

(a) first floor (dof 1) and b) roof (dof 3), of the model adopted in discrete event example. 


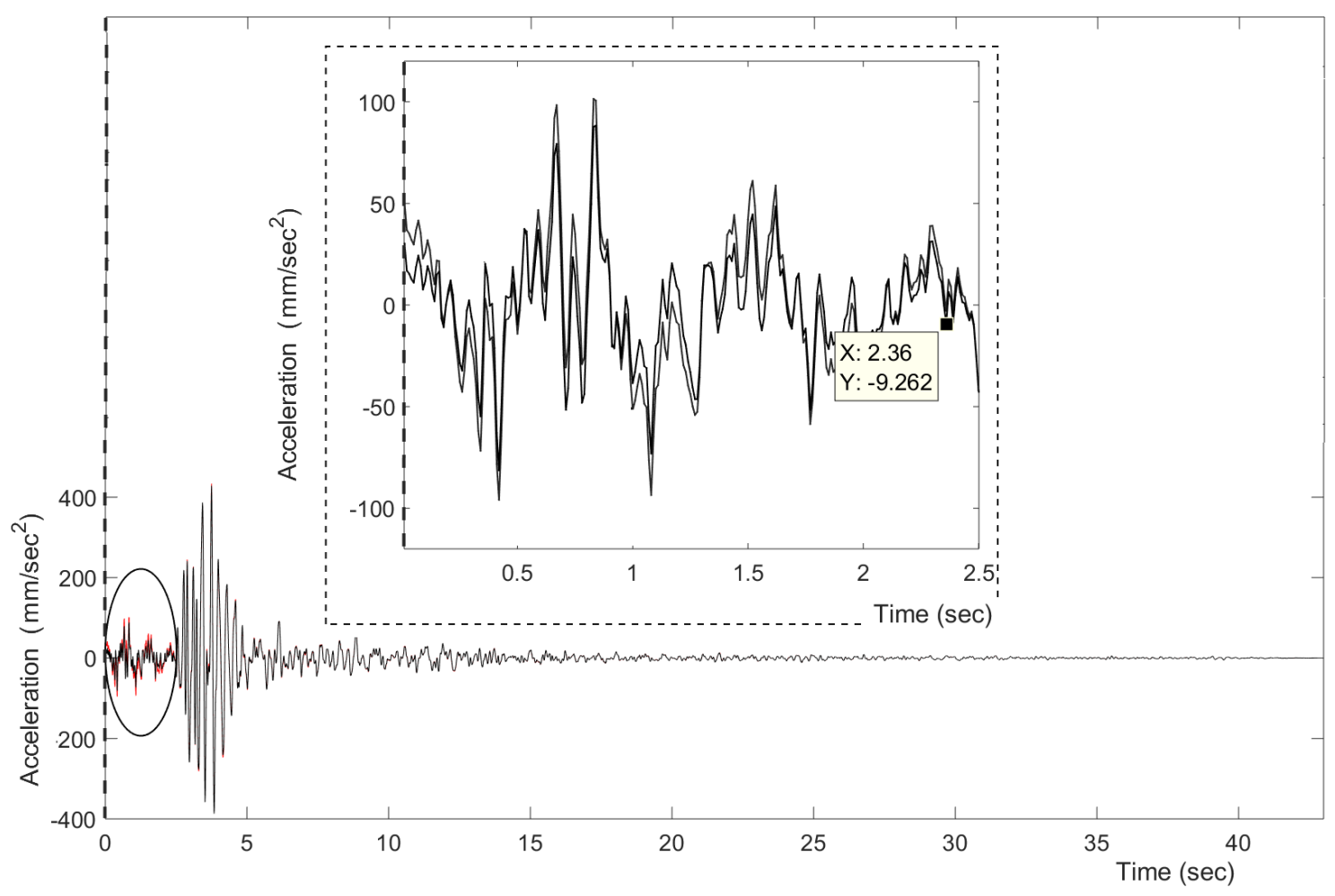

Figure 2.12. Comparison of the exact response and the response simulated by Pseudo load at first floor of the model adopted in discrete event example. 


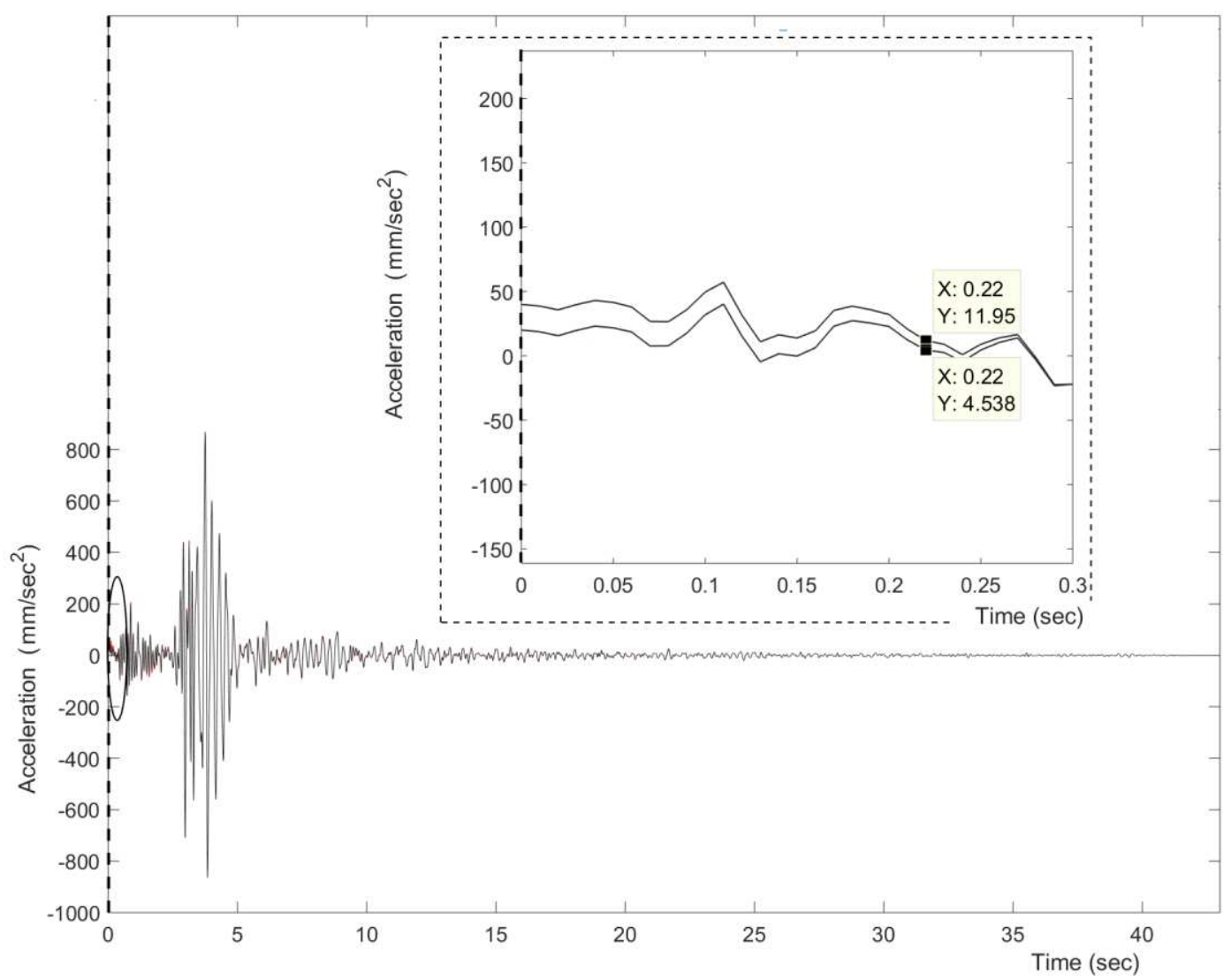

Figure 2.13. Comparison of the exact response and the response simulated by Pseudo load at roof level of the model adopted in discrete event example 

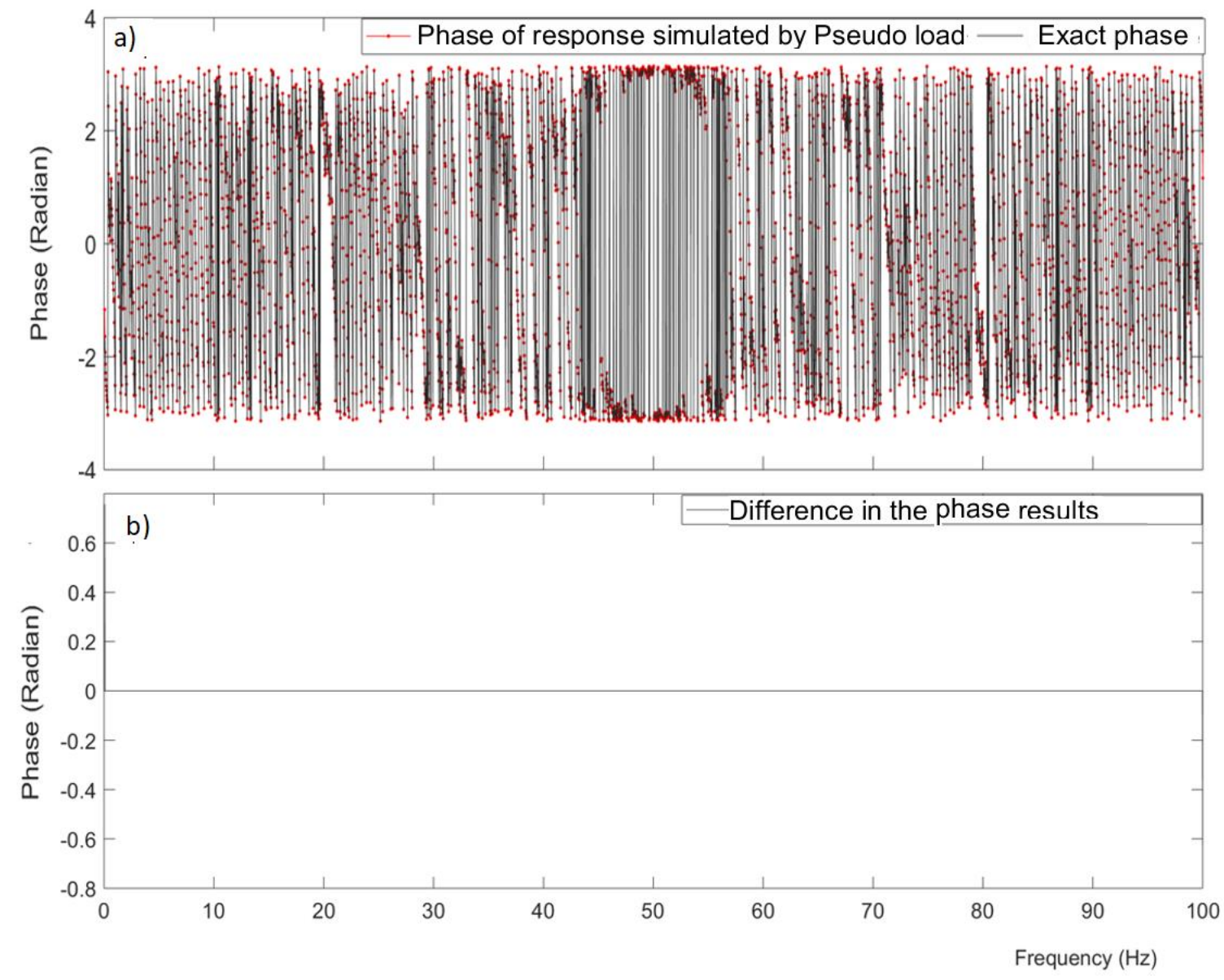

Figure 2.14. Comparison of the exact phase and the phase obtained from response simulated by Pseudo load in the model adopted in discrete event example, at first floor (b) difference between the exact phase and the phase obtained from response simulated by Pseudo load. 

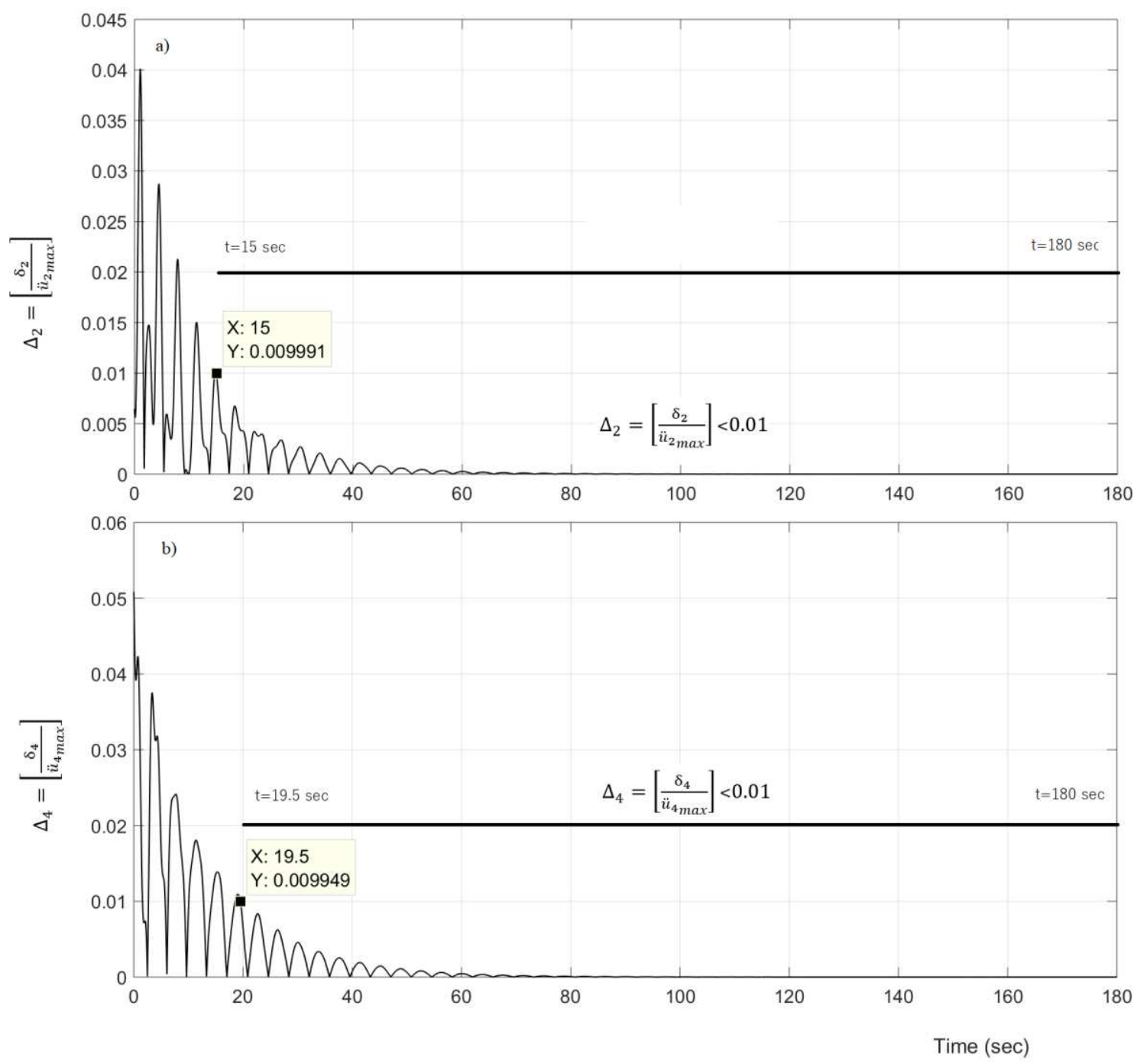

Figure 2.15. Normalized relative error in the response simulated by Pseudo load in the ambient scenario example at; (a) mid height (dof 2) and b) roof (dof 4). 


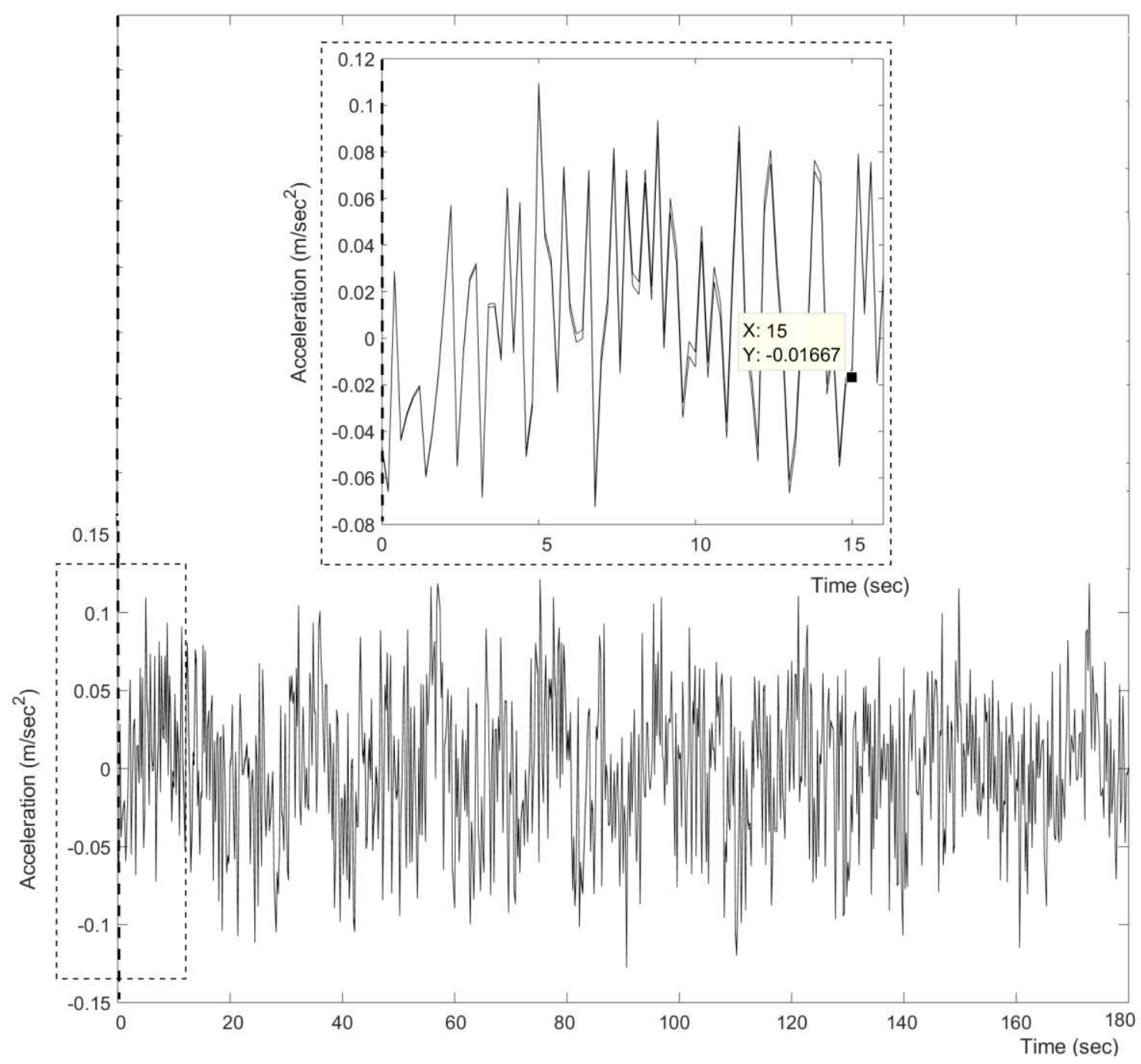

Figure 2.16. Comparison of the exact response and the response simulated by Pseudo load at the second floor in the ambient scenario example. 


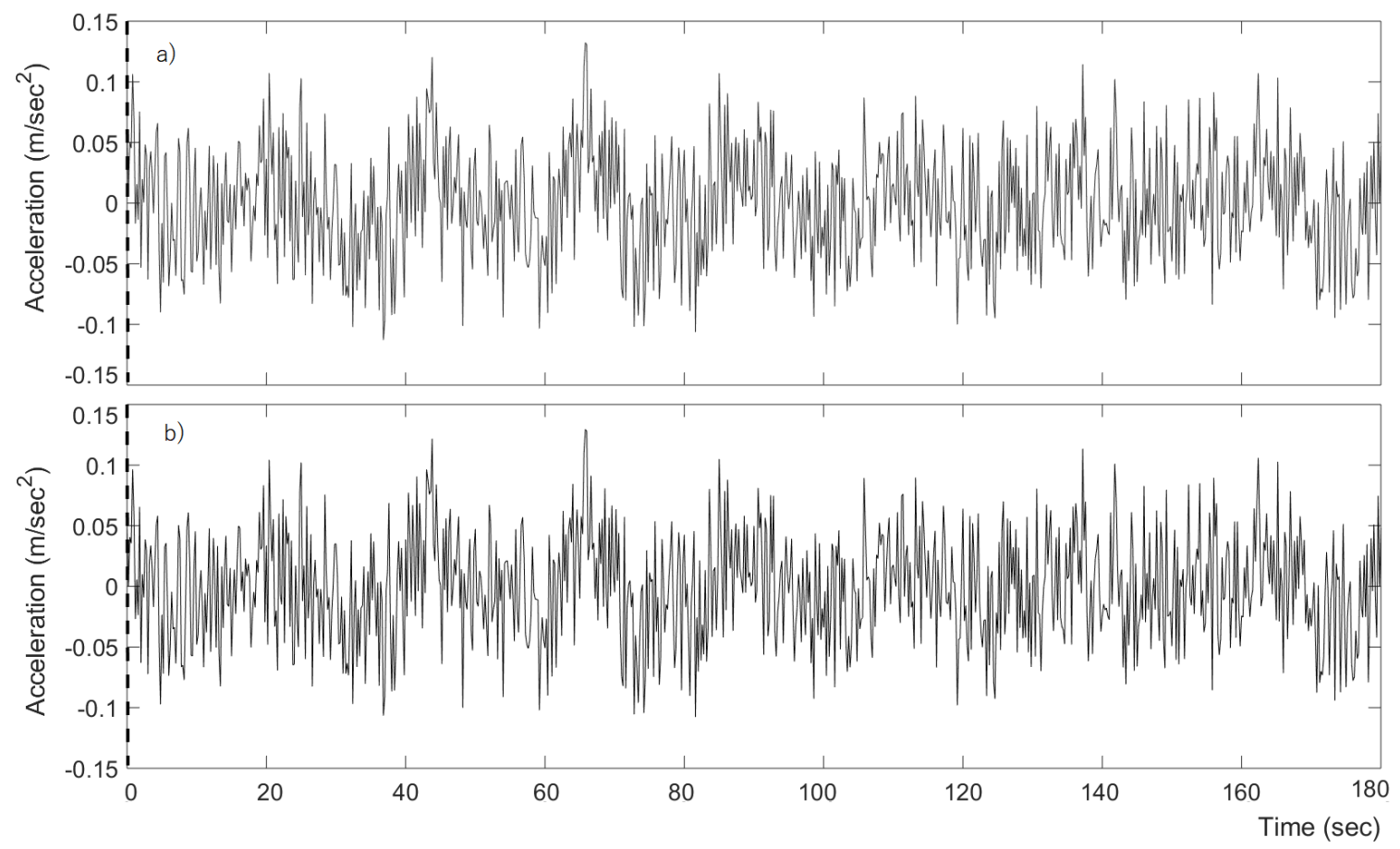

Figure 2.17. Comparison of (a) the exact response and (b) the response simulated by Pseudo load at the roof level in the ambient scenario example. 

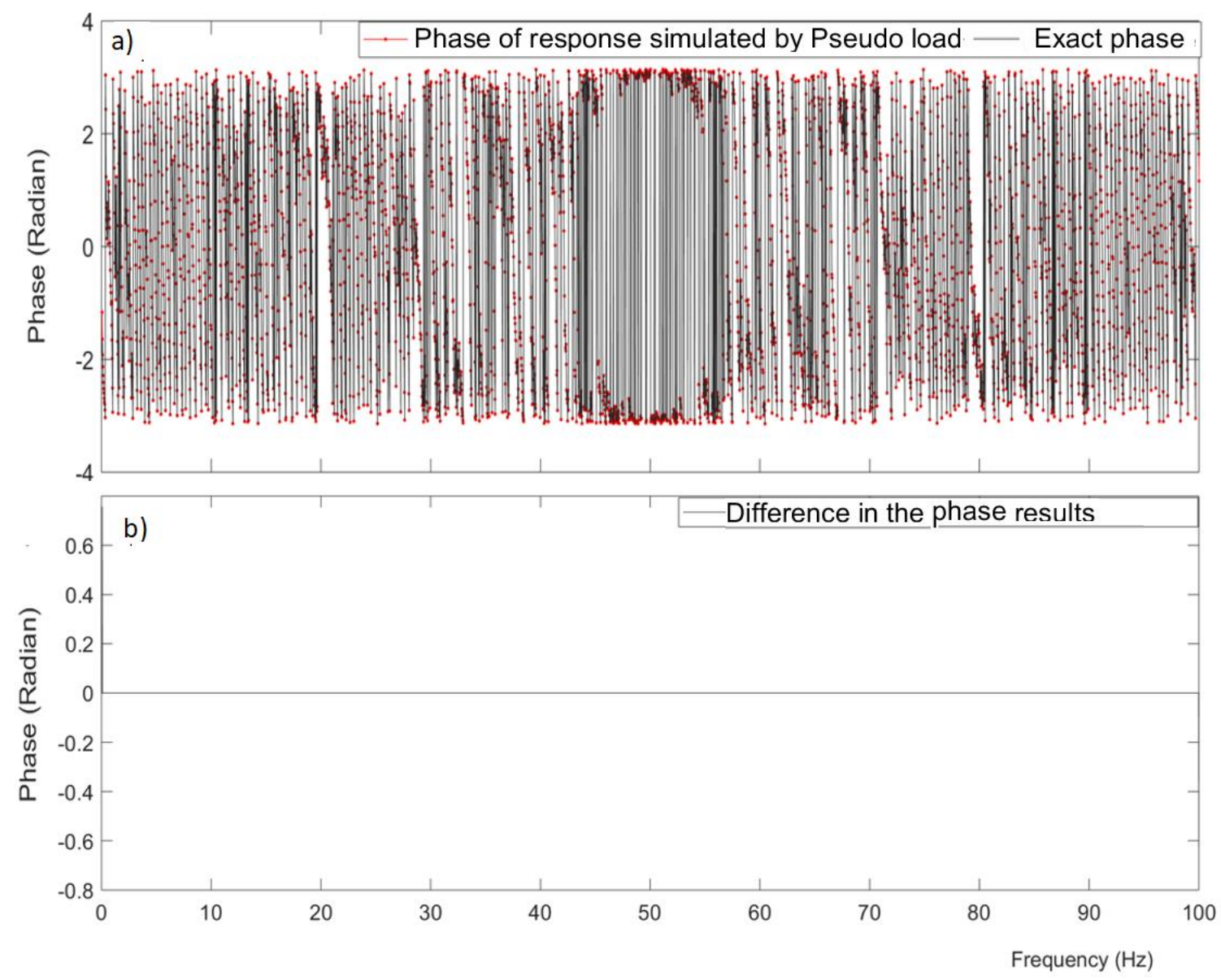

Figure 2.18. (a) Comparison of the exact phase and the phase obtained from response simulated by Pseudo load in the ambient scenario example, at roof level (b) difference between the exact phase and the phase obtained from response simulated by Pseudo load. 
Table 2.1 Geometric and material properties of the model in ambient scenario example

\begin{tabular}{|l|c|}
\hline Height of each column $(\mathrm{mm})$ & 5000 \\
\hline Modulus of Elasticity $\left(\mathrm{N} / \mathrm{mm}^{2}\right)$ & 200000 \\
\hline Moment of inertia $\left(\mathrm{mm}^{4}\right)$ & 5208333 \\
\hline
\end{tabular}


Table 2.2 Initial conditions in the ambient scenario example

\begin{tabular}{|l|c|c|c|c|}
\hline Initial conditions & First floor & Second floor & Third floor & Roof \\
\hline Displacement $(\mathrm{m})$ & 0.006 & 0.0121 & 0.015 & 0.019 \\
\hline Velocity $(\mathrm{m} / \mathrm{s})$ & 0.0045 & 0.0079 & -0.0055 & 0.0061 \\
\hline
\end{tabular}


Table 2.3 Geometric and material properties of the model in discrete event example

\begin{tabular}{|l|c|}
\hline Height of base column $(\mathrm{mm})$ & 3801 \\
\hline Height of remaining columns $(\mathrm{mm})$ & 3408 \\
\hline Modulus of elasticity $\left(\mathrm{N} / \mathrm{mm}^{2}\right)$ & 200000 \\
\hline Moment of inertia $\left(\mathrm{mm}^{4}\right)$ & 467229.7 \\
\hline
\end{tabular}


Table 2.4 Initial conditions in the discrete event example

\begin{tabular}{|l|c|c|c|}
\hline Initial conditions & First floor & Second floor & Roof \\
\hline Displacement $(\mathrm{mm})$ & 20 & 15 & 25 \\
\hline Velocity $(\mathrm{mm} / \mathrm{sec})$ & 20 & -30.3 & 35 \\
\hline
\end{tabular}




\section{Chapter 3. Case Study of Simulation of Damage Vibration Response}

\subsection{Summary}

Due to their advantages in comparison to other structural assessment methodologies, vibration based structural health monitoring (VBSHM) techniques have gained increasing research attention in recent years and are now the basis of the development of many damage detection methods for civil engineering structures, especially for structures operating under harsh environments like the Confederation Bridge in Canada. Vibration based structural health monitoring methods implemented in continuous monitoring applications of instrumented structures allow for efficient structural condition assessment or damage detection based on observation and evaluation of the extracted field observed global behaviour and performance of the structure. The VBSHM structural condition assessment and damage detection methodologies offer the best chance of early detection of abnormalities so that timely repair and maintenance requirements can be carried out to ensure public safety and minimize the financial burden on the custodian authorities of public and private infrastructure. Studies by Desjardins et al. (2006), Londoño et al. (2013), etc., have shown the influences of variabilities in the operational and environmental conditions on the vibration responses of structures in the field. This can have significant impact to the effectiveness of VBSHM methods to solve problems of real structures in the field. Recognizing this limitation of VBSHM methodologies for damage detection of structures in the field, it is important to realize that most of the previous investigation (Sohn et al. 2003 and Kullaa 2003) on the influence of noise and uncertainties of VBSHM based damage detection techniques were either tested on simulated damage data that lack the true 
noise and uncertainties from the field or were developed and tested on structures in controlled laboratory conditions that altogether were deprived of the field noise and uncertainties resulting from the operating conditions of the structure, such as variabilities in the boundary conditions, material properties, loading conditions, as well as that of the environment, such as temperature, wind and traffic conditions etc. The concept of Pseudo load presented in Chapter 2 is applied to simulate damage vibration response of monitored structures with realistic field recorded noise and uncertainties inherent in each field captured vibration response monitoring dataset. In this chapter, the Pseudo load procedure is applied to simulate damage vibration time history response of the Confederation Bridge in Canada for various damage scenarios using a finite element model of the Confederation Bridge developed in SAP2000 and field captured vibration response data from the Confederation Bridge monitoring system.

\subsection{Introduction}

Safe operation of civil infrastructure like highway bridges has always been a high priority in civil engineering practice and for a long time has relied on regular periodic visual inspection or emergency inspection after the occurrence of discrete extreme events, such as earthquakes or windstorms. Despite the existing visual based monitoring practice in infrastructure safety and risk management has proven to be able to keep structures safe, its limitation in detecting early sign of structural deterioration and results that are subjective and dependent on the judgement and experience of the inspector, the localized assessment is insufficient to quantify the overall global structural performance and its integrity. 
Therefore, there is the need for improvement in current practices of structural assessment. With the recent advances in internet and telecommunication technologies, vibration based structural health monitoring (VBSHM) methodologies are gradually being accepted as a possible viable alternative to the current visual inspection based practice of structural condition assessment. The advantages of VBSHM are even more pronounced for structures with high socio-economic importance especially when they are located in harsh environment like the Confederation Bridge in Canada. The basic premise of VBSHM methodologies for determining/quantifying structural health is that vibration properties or characteristics of a structure such as its natural frequencies, mode shapes and damping properties are indicators of conditions or states of the structure, and any deviation from a standard reference state is associated with a change in the structural condition, which can be interpreted as deterioration or damage. One of the fundamental reasons of increasing acceptance of VBSHM methodologies in development of more sophisticated structural condition assessment tools is that VBSHM methodologies do not require structural testing in the field to collect data for analysis. Vibration based structural health monitoring methods can use vibration response data captured under random ambient loadings such as from wind and traffic as the sources of excitation which are can be collected anytime, more economically and efficiently, and do not lead to service disruptions. The development of VBSHM techniques has received increasing attention in the past three decades as evident from the literature. Research from the basic idea of comparing the dynamic characteristics by experiments on simple structural members (Cawley and Adams 1979) to more rigorous methods like finite element updating (Teughels et al. 2002, Londoño et al. 2013 and Rahman et al. 2013) and statistical pattern recognition (Tehranian et al. 2002) have been 
carried out. From the practical point of view, the vibration based structural health monitoring faces various challenges including the noise and uncertainties in data arising from variabilities in the environment, loading and operational conditions, which can obscure the effects of damage or deterioration of the monitored structure (Desjardins et al. 2006, Humar et al. 2006 and Londoño et al. 2013). In the review of previous studies of VBSHM, existing VBSHM techniques developed for detecting damage so far either ignore or inadequately consider the effect of field noise and uncertainties; or do not account for the realistic uncertainty effects in testing the effectiveness and efficiency of the methodologies. The method of Pseudo load discussed in Chapter 2 is applied here to simulate damage response of a structure with realistic field noise and uncertainties. The realistic simulated damage response can then be used to test the effectiveness of existing VBSHM methods or may be used as crucial data necessary for the development of more robust and reliable VBSHM methods and tools for practical real world applications of the technologies. Each recorded vibration response dataset has unique noise and uncertainties embedded in the data from the field at the time of the data capture. Consequently, the use of the Pseudo load as input excitation for generating the simulated damage response will have the identical uncertainties in the damage response as in the original dataset without the need of development and adoption of an uncertainty model which inherently would not be as accurate compared to the actual noise and uncertainties directly recorded in the field. The effects of the unknown initial conditions are removed from the simulated damage response obtained by the proposed Pseudo load method making the simulated result to become exact. In this chapter, the Pseudo load procedure presented in Chapter 2 is adapted by the substructuring technique to simulate realistic damage response of the Confederation 
Bridge. A finite element model of the Confederation Bridge developed in SAP2000 and field captured ambient response data are used.

\subsection{Vibration Monitoring of Confederation Bridge}

\subsubsection{General}

Designed for a service life of 100 years, the Confederation Bridge is a $12.9 \mathrm{~km}$ long multispan post-tensioned concrete box girder curved bridge between Prince Edward Island (P.E.I) and New Brunswick (N.B), in eastern Canada. It consists of 43 spans, each 250 meters in length with 2 end spans $165 \mathrm{~m}$ each. The total width of the bridge is 12 meters, and it comprises of the main bridge in the middle and two approach bridges which are built of precast concrete segments assembled by post-tensioned tendons.

\subsubsection{Monitoring system}

The Confederation Bridge vibration monitoring system has 76 accelerometers distributed along one typical portal frame unit between piers P30 and P33. It continuously records the output only vibration response of the bridge in lateral, longitudinal and vertical directions, as shown in Fig. 3.1, as per user defined sampling rate which varies from $100 \mathrm{~Hz}$ to 167 Hz. After onsite filtering and conditioning of the voltage signals from the sensors for antialiasing using 8-pole $50 \mathrm{~Hz}$ low-pass Bessel filter, the signals are subjected to analog to digital conversion (A/D) by three data loggers located on the bridge, which are programmed to collect data from sensors either on continuous basis or in case of triggering event. Onsite and remote computers control and operate the data loggers for remote 
automatic data retrieval for real-time processing or archival to a centralized platform for later use, where the data are accessible to researchers for analysis. For the ongoing research, the monitoring data from three data loggers on the bridge are automatically transmitted to Carleton University by high-speed internet for data processing and analysis and interpretation (Desjardins 2006).

\subsubsection{Data Processing by Graphic User Interface (GUI)}

The data to be analyzed is first processed by a graphic user interface algorithm with data management, analysis and visualization modules that have been specially developed for detecting the potential problems in the Confederation Bridge monitoring data and automatically repair them (Desjardins 2004). The algorithm first identifies and separates the different data events and assembles any matching segment to form a complete set of specified data event of proper duration and then synchronizes all the data event from all the data loggers that correspond to same dynamic event forming a dataset. Data processing module performs various operations on the data that includes patching of the data set in case of missing data, purging any duplicate data, decimation of data set to adjust the sampling rate as per frequency of interest, detrending of the recorded acceleration datasets to remove drift effects, double integration to obtain displacement responses etc. 


\subsection{Damage Simulations}

\subsubsection{Methodology}

The vibration response of a structure subjected to the field conditions can be derived using Eq. 2.1 with updated structural properties of mass $[\mathrm{M}]$, damping $[\mathrm{C}]$, and stiffness $[\mathrm{K}]$ matrices by model updating using information extracted from the acceleration response data $\ddot{u}_{n}$ measured from the field. If the initial conditions of the structure are known, the velocity $\dot{u}_{n}$ and displacement $u_{n}$ response can be calculated by numerical integration of the acceleration response. The computed ambient load $F_{n}$, as shown in Eq. 2.1, can then be used to generate an exact damage response with identical field noise and uncertainties as in the original dataset of intact state by applying the load to a finite element model of the simulated damage state of the structure. However, in most VBSHM systems, only acceleration responses are recorded, and the initial conditions of the monitored structure are generally not known. It is therefore generally not possible to exactly determine the velocity and displace time history responses of the structure without knowing the initial conditions of the monitored structure. The alternative in this case is to calculate the velocity and displacement time history responses without the effects of the initial conditions, i.e. considering the case of zero initial conditions. The load computed using the recorded acceleration response $\ddot{u}_{n}$ and numerically calculated velocity $\dot{\tilde{u}}_{n}$ and displacement $\tilde{u}_{n}$ responses with zero initial conditions, as shown in Eq. 2.6, is defined as the Pseudo load $P_{\text {Pseudo }}$. The Pseudo load is then applied to a finite element model modified to represent the damaged structure to simulate the damaged response of the structure. By comparing this simulated damage response using the Pseudo load with that of the exact damage 
response using the initial conditions of the structure, the influence of the initial conditions, as given by Eq. 2.17, on the simulation results can be evaluated.

\subsubsection{Pseudo Load for Monitored Structures}

The definition of Pseudo load from Chapter 2, as shown in Eq. 2.6 is very restrictive and is applicable to monitored structures where the number of sensors installed $n$ match with the number of degrees-of-freedom $m$ of its finite element model. In most SHM systems, the number of sensors is typically significantly less than the total number of degrees-offreedom $m$ of the finite element model of the monitored structure. In the discussion here, the subscript $\mathrm{s}$ denotes responses at the sensor locations and the subscript $\mathrm{d}$ denotes responses at the dof's of the finite element model where the responses are not captured by sensors.

If $\ddot{u}_{S}, \dot{\tilde{u}}_{s}, \tilde{u}_{s}$ represent the measured responses from the $\mathrm{s}$ number of installed sensors corresponding to the $\mathrm{s}$ dof's in the finite element model of the monitored structure, $\ddot{u}_{d}, \dot{\tilde{u}}_{d}, \tilde{u}_{d}$ denote the unknown responses at the remaining $\mathrm{d}$ dof's of the monitored structure where there are no sensors. The equation of motion of the monitored structure can be expressed as follows

$$
\begin{gathered}
{\left[\begin{array}{ll}
M_{s s} & M_{s d} \\
M_{d s} & M_{d d}
\end{array}\right]\left\{\begin{array}{c}
\ddot{u}_{s} \\
\ddot{u}_{d}
\end{array}\right\}+\left[\begin{array}{ll}
C_{s s} & C_{s d} \\
C_{d s} & C_{d d}
\end{array}\right]\left\{\begin{array}{c}
\dot{\tilde{u}}_{s} \\
\dot{\tilde{u}}_{d}
\end{array}\right\}+\left[\begin{array}{ll}
K_{s s} & K_{s d} \\
K_{s d} & K_{d d}
\end{array}\right]\left\{\begin{array}{c}
\tilde{u}_{s} \\
\tilde{u}_{d}
\end{array}\right\}} \\
=\left\{\begin{array}{c}
P_{s s} \\
P_{s d}
\end{array}\right\}+\left\{\begin{array}{l}
P_{d s} \\
P_{d d}
\end{array}\right\}
\end{gathered}
$$

Equation 3.1 can be separated into Eqs. 3.2 and 3.3 based on monitored and nonmonitored dof's as follows 


$$
\begin{gathered}
{\left[\begin{array}{cc}
M_{s s} & M_{s d} \\
M_{d s} & M_{d d}
\end{array}\right]\left\{\begin{array}{c}
\ddot{u}_{s} \\
0
\end{array}\right\}+\left[\begin{array}{cc}
C_{s s} & C_{s d} \\
C_{d s} & C_{d d}
\end{array}\right]\left\{\begin{array}{c}
\dot{\tilde{u}}_{s} \\
0
\end{array}\right\}+\left[\begin{array}{cc}
K_{s s} & K_{s d} \\
K_{s d} & K_{d d}
\end{array}\right]\left\{\begin{array}{c}
\tilde{u}_{s} \\
0
\end{array}\right\}=\left\{\begin{array}{c}
P_{s s} \\
P_{s d}
\end{array}\right\}} \\
{\left[\begin{array}{cc}
M_{s s} & M_{s d} \\
M_{d s} & M_{d d}
\end{array}\right]\left\{\begin{array}{c}
0 \\
\ddot{u}_{d}
\end{array}\right\}+\left[\begin{array}{ll}
C_{s s} & C_{s d} \\
C_{d s} & C_{d d}
\end{array}\right]\left\{\begin{array}{c}
0 \\
\dot{\tilde{u}}_{d}
\end{array}\right\}+\left[\begin{array}{ll}
K_{s s} & K_{s d} \\
K_{s d} & K_{d d}
\end{array}\right]\left\{\begin{array}{c}
0 \\
\tilde{u}_{d}
\end{array}\right\}=\left\{\begin{array}{l}
P_{d s} \\
P_{d d}
\end{array}\right\}}
\end{gathered}
$$

The right-hand side of Eq. 3.2 is defined as the Pseudo load which is evaluated by the lefthand side of Eq. 3.2 where all the parameters are known. This calculated Pseudo load when used as input excitation back in the finite element model of Eq. 3.1 will satisfy Eq. 3.3. This indicates that when the Pseudo loads calculated from the assumed intact finite element model are used as input excitations at the corresponding $m$ dof's, it will result in zero responses at the nodes where there are no sensor and field recorded responses are unavailable.

The Pseudo load when applied to damaged state of the structure's finite element model gives the damaged response of the structure with identical set of field noise and uncertainties as present in the original intact state. For all other remaining degrees of freedom corresponding to those locations where there are no sensors, the response in the damaged state would not be zero, as according to Eq. 3.2 with different mass and damping matrices for the damaged state. As such, any deviation from the ideal state in itself could be considered as indicator of the error in the simulated damage response at the sensor locations.

\subsubsection{Simulation of Damage Scenarios}

To get an estimate of the effect of the unknown initial conditions in damage simulations using the Pseudo loads, the updated finite element model of the structure is subjected to 
the calculated Pseudo loads, as given by Eq. 3.2 to obtain the damage responses. The responses at an arbitrary chosen instant in the time history responses of the structure are selected as the initial conditions of the structure for calculating the ambient load given in Eq. 3.4.

$$
\left[\begin{array}{ll}
M_{s s} & M_{s d} \\
M_{d s} & M_{d d}
\end{array}\right]\left\{\begin{array}{c}
\ddot{u}_{s} \\
0
\end{array}\right\}+\left[\begin{array}{cc}
C_{s s} & C_{s d} \\
C_{d s} & C_{d d}
\end{array}\right]\left\{\begin{array}{c}
\dot{u}_{s} \\
0
\end{array}\right\}+\left[\begin{array}{cc}
K_{s s} & K_{s d} \\
K_{s d} & K_{d d}
\end{array}\right]\left\{\begin{array}{c}
u_{s} \\
0
\end{array}\right\}=F_{n}
$$

For typical civil engineering structures, the effect of the initial conditions is limited to a relatively short time of the vibration response due to damping behaviour of the structures. For damage simulation purposes, a portion of the Confederation Bridge between piers 29 and 32 which includes two consecutive portal frames plus the expansion drop-in span, a reasonable representation of the geometric and dynamic characteristics of the bridge (Londoño and Lau 2003), is chosen as the test structure, as shown in Fig. 3.3. The finite element model of the test structure is modelled by SAP 2000 software based on information obtained from the construction drawing specifications of the bridge. The model consists of a total of 123 3D beam elements, 124 nodes, as shown in Fig. 3.3. The mass density of the nonstructural components like road barriers and pavements is included in the mass of the structural components. Furthermore, the finite element model is one of the models that has been calibrated using finite element updating technique by Londoño (2004) to match the field measured responses.

Two damage scenarios are considered for simulation: (i) degradation of piers potentially arising due to long term immersion in saltwater; and (ii) localized damage of posttensioning prestressed tendons and grouting at the joints of the drop-in spans due to gradual 
pretensioning loss or tendon failure resulting in partial loss in continuity and in stiffness at the joints.

Scenario 1: Degradation of Piers

This assumed damage scenario due to corrosion damage of the piers exposed to sea water resulting in cracking and stiffness reduction of the Piers $29,30,31,32$ is modelled by reducing of the stiffness of the structural elements of the piers below sea level by $3 \% 5 \%$ and $10 \%$.

Scenario 2: Damage of drop-in span joints

This assumed damage scenario that may result in the loss of prestressing /stiffness in the tendons is modelled by reducing the stiffness of the structural elements over the length of the drop-in span joints by $3 \% 5 \%$ and $10 \%$.

For simulating the damage response of the confederation bridge with field noise and uncertainties using Pseudo loads, a dataset is selected from the Confederation Bridge monitoring system under ambient loading scenarios. Out of the 18 transverse acceleration time histories, recorded by the 18 permanently installed accelerometers between piers 31 and 32 present in the selected dataset acceleration data at channels 1 and 3 , as shown in Fig. 3.4, and channels 8 and 9, as shown in Fig. 3.5, are chosen for simulation.

The dataset has been selected to ensure that they strictly represent the ambient loading scenarios. The data set is processed using monitoring software developed for the Confederation Bridge project by Desjardins et al. (2006) to perform tasks like removing drift from acceleration voltage signals, down sampling of the data to one third of the original sampling rate and eliminating high frequency noises and signal components. 
For pier damage scenario out of the total 124 nodes of the finite element model of the bridge, only the recorded vibration response at the two locations of sensors 1 and 3 are used for the simulation here. The remaining 122 nodes are assumed to have no sensors. For the case of the drop-in damage scenario out of a total 124 nodes of the finite element model of the bridge, the responses recorded at sensors 8 and 9 are used for the simulation here. The remaining 122 nodes are assumed to lack sensors.

\subsubsection{Pier Damage Scenarios}

From the entire captured response of 10-minute duration, the initial 100 seconds are chosen for simulations. To account for the effect of initial conditions, using the finite element model of the bridge and the recorded responses, first the Pseudo loads are calculated using Eqs. 3.2 and 3.5.

For pier damage scenario, recorded responses at sensors 1 and 3 are used.

$$
\ddot{u}_{s}=\left[\begin{array}{l}
\ddot{u}_{1} \\
\ddot{u}_{3}
\end{array}\right]
$$

where $\ddot{u}_{1}$ and $\ddot{u}_{3}$ are the recorded transverse acceleration response at sensors 1 and 3 , respectively with a duration of 100 secs. The corresponding velocity $\dot{\tilde{u}}_{s}$ and displacement $\tilde{u}_{s}$ responses are obtained by integration of the acceleration signals using SPLASH (Desjardins 2004), the vibration data processing and analysis software platform developed for the Confederation Bridge. The dynamic responses at $\mathrm{t}=10$ seconds are assumed to be the initial responses of the structure with the initial conditions shown in Table 3.1.

The dynamic load calculated using Eqs. 3.4 and 3.5 with zero initial conditions and the Pseudo loads calculated using Eqs. 3.2 and 3.5 beyond $t=10$ seconds are then used to simulate the exact and simulated damage responses for various damage scenarios. 


\subsubsection{Drop-in Span Joint Damage Scenarios}

For drop-in damage scenario, Pseudo loads, and dynamic loads are calculated using Eqs. 3.2, 3.4 and 3.6 following the same derivation procedure as presented in Section 3.4.3.1, to simulate the vibration damage responses at sensors 8 and 9 ,

$$
\ddot{u}_{s}=\left[\begin{array}{l}
\ddot{u}_{8} \\
\ddot{u}_{9}
\end{array}\right]
$$

\subsection{Discussion on Simulated Damage Responses}

Figure 3.6 shows the normalized relative error of the simulated damage response at the sensor 1 for the cases of $3 \%$ and $10 \%$ damage level in the piers. For the two damage levels, it is found that the simulated damage responses satisfy the criterion of Eq. 2.19 at $9.02 \mathrm{sec}$ and $11.81 \mathrm{sec}$ respectively of the total duration of 100 seconds. Figures 3.7 and 3.8 shows the comparisons between exact and Pseudo load simulated damage responses for 3\% and $10 \%$ damage levels respectively. The difference diminishes rapidly after the first few seconds. Considering only the responses after 11.812 .20 for $10 \%$ damage in the piers, Fig. 3.9 shows that there is hardly any difference between the phase of the simulated damage response and the exact damage response.

Figure 3.10 shows the normalized relative error of the simulated damage response at the sensor 8 for $3 \%$ and $10 \%$ damage levels in the drop-in span joint. It is found that the simulated damage responses satisfy the criterion of Eq. 2.19 at $11.81 \mathrm{sec}$ and $13.84 \mathrm{sec}$ for $3 \%$ and $10 \%$ damage levels respectively. Figures 3.11 and 3.12 shows the comparison between the exact and the Pseudo load simulated damage responses for the 3\% and $10 \%$ damage levels respectively. The difference again diminishes rapidly after the first few seconds. Considering only the responses after $13.84 \mathrm{sec}$ according to the criterion of Eq. 
2.19 for the case of 10\% damage in the drop-in span joint. Again Fig. 3.13 shows that there is hardly any difference between the phase of the simulated damage response and the exact damage response.

Figures 3.14 and 3.15 show the captured response and the difference between the captured response and the simulated responses for various damage levels in the piers at sensor 1 and drop-in span joint at sensor 8 respectively. It can be seen that the difference increases with the level of damage. Figure 3.16 shows the power spectral density up to $20 \mathrm{~Hz}$ of the captured response at sensor 1, and that of the simulated responses with $3 \%$ and $10 \%$ damage in the piers. It can be observed that the peak shifts towards lower frequency as the level of damage increases. The three cases have similar power density distribution, which indicates that the Pseudo load method is able to simulate the response of damaged structure preserving same noise and uncertainties as present in the captured response. Since both captured data and simulated damage data have same noise and uncertainties, the difference in power spectral densities is clearly due to damage alone, which has never been demonstrated in previous VBSHM research. Figures 3.17 to 3.19 show the power spectral densities of the captured response at sensor 8 , and that of the simulated responses with 3\% and $10 \%$ damage at the drop-in span joint. Similar to the observations in the case of pier damage scenario, the power spectral densities of captured response and the response simulated by Pseudo load are similar, indicating same noise and uncertainties in both the captured response data set and simulated response data set. The absolute difference between the power spectral densities of the captured response and the simulated responses for the 3\% and 10\% damage cases in the drop-in span joint are shown in Figs. 3.20 and 3.21 respectively. The difference is small since the damage level is relatively low. 

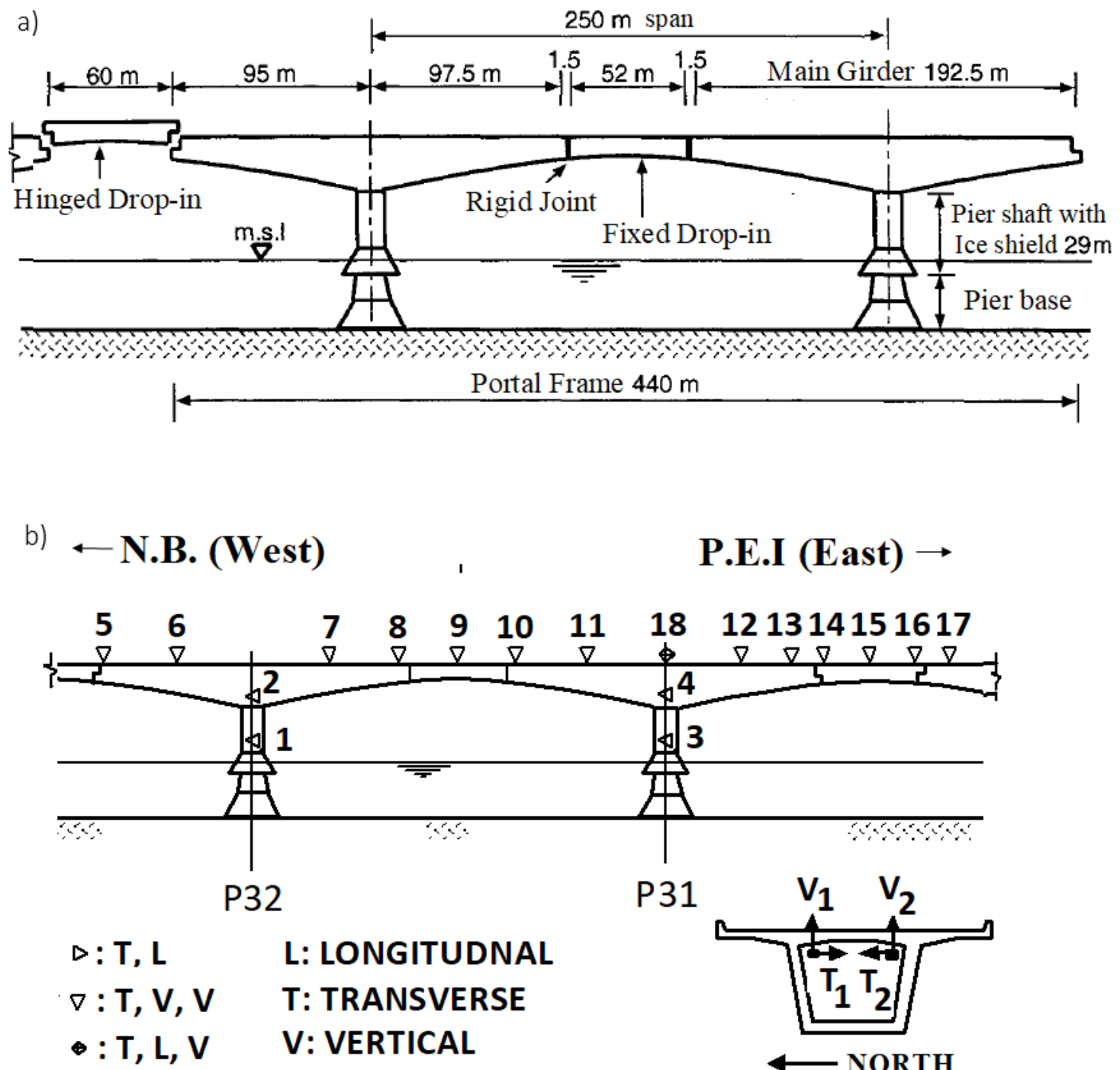

P.E.I (East) $\rightarrow$

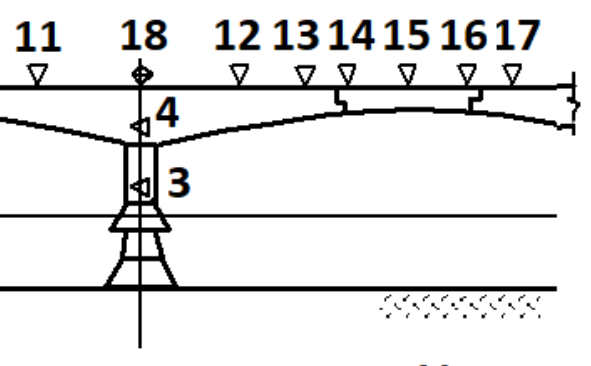

P31

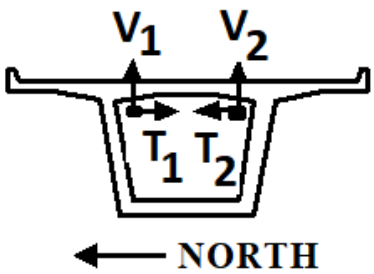

Figure 3.1. (a) Dimensions and main component of typical structural module span (b) locations of 50 accelerometers out of the total 76 installed in Confederation Bridge monitoring system. 


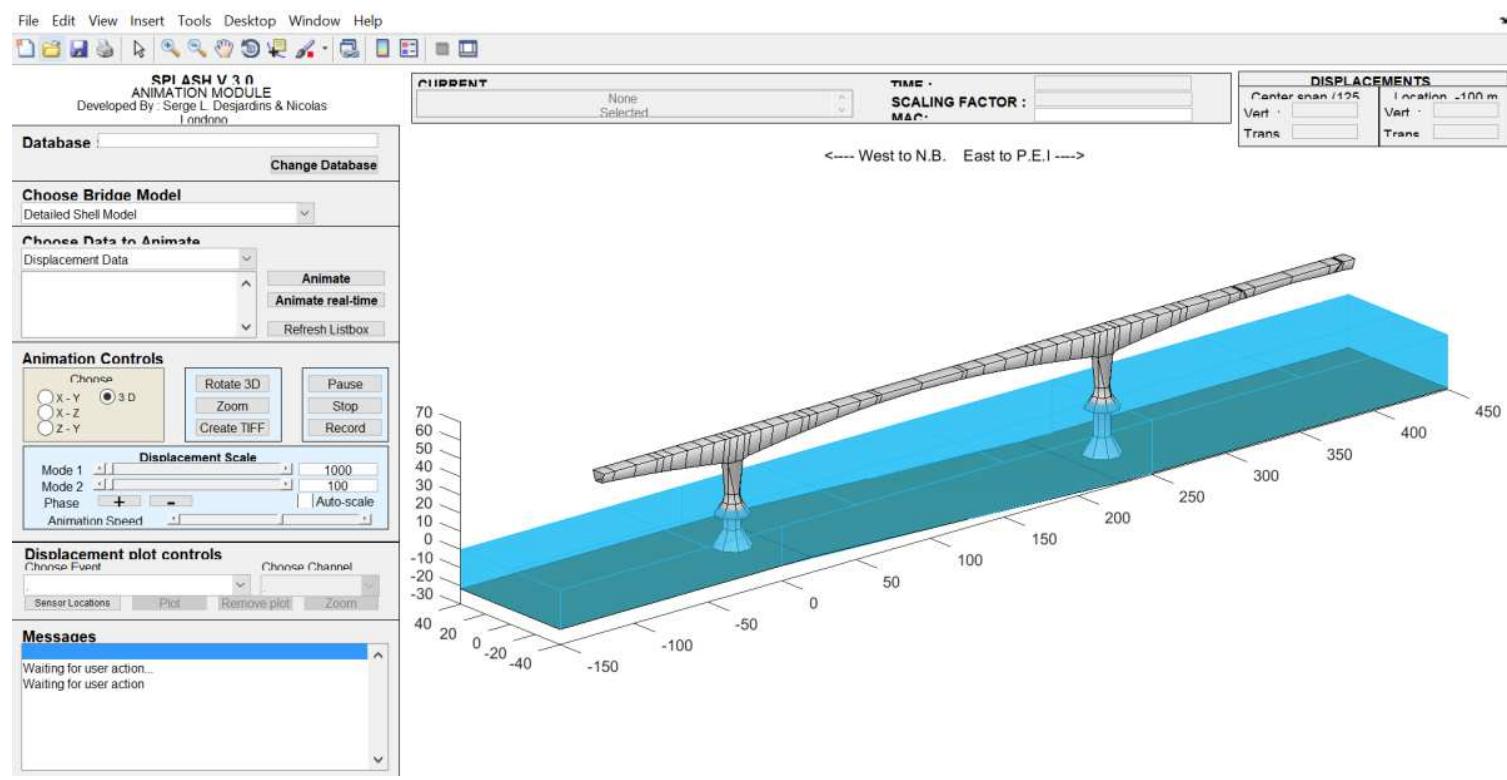

Figure 3.2. Visualization module of GUI developed for the Confederation Bridge taken from the SPLASH algorithm developed by Desjardins (2004). 
(a)

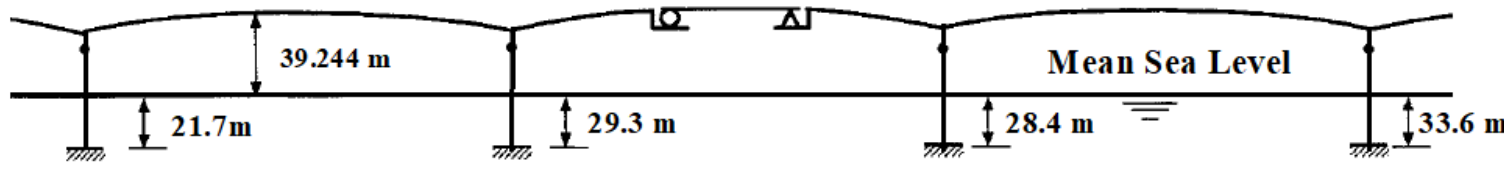

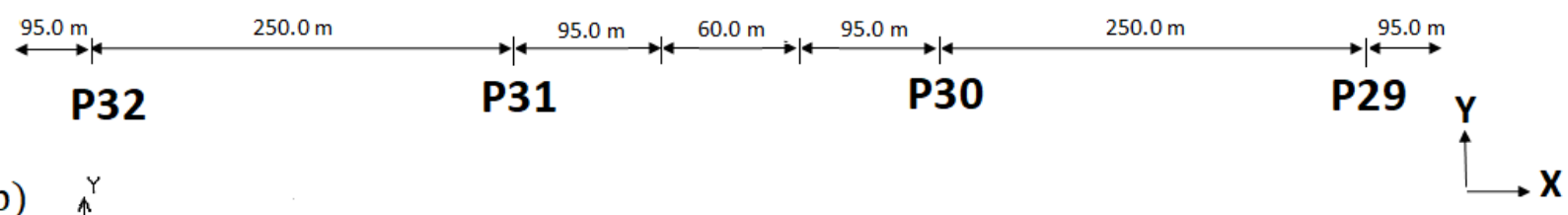

(b)

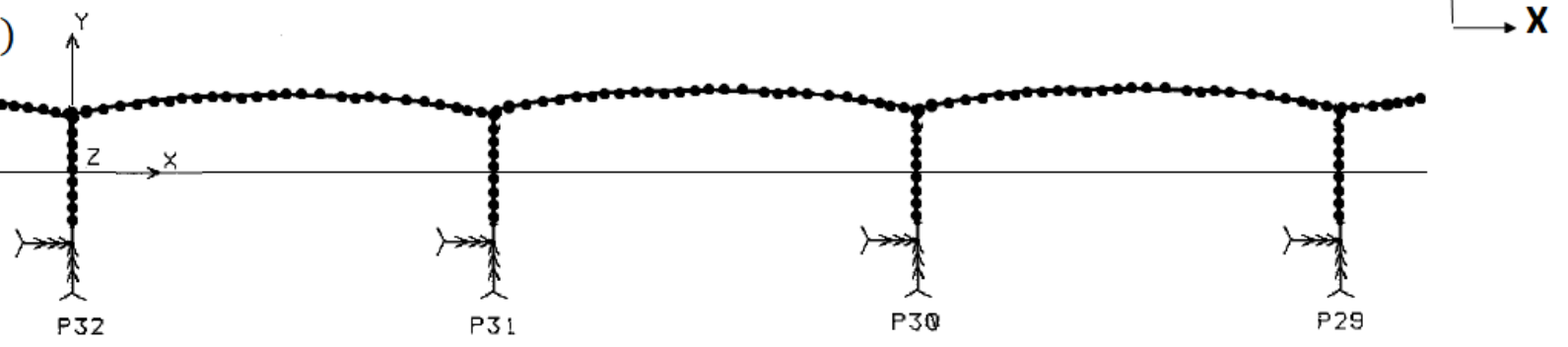

Figure 3.3. (a) Test structure of Confederation Bridge used for demonstrating the proposed methodology (b) beam element model of the test structure schematic showing mesh from the study of Londoño (2004). 

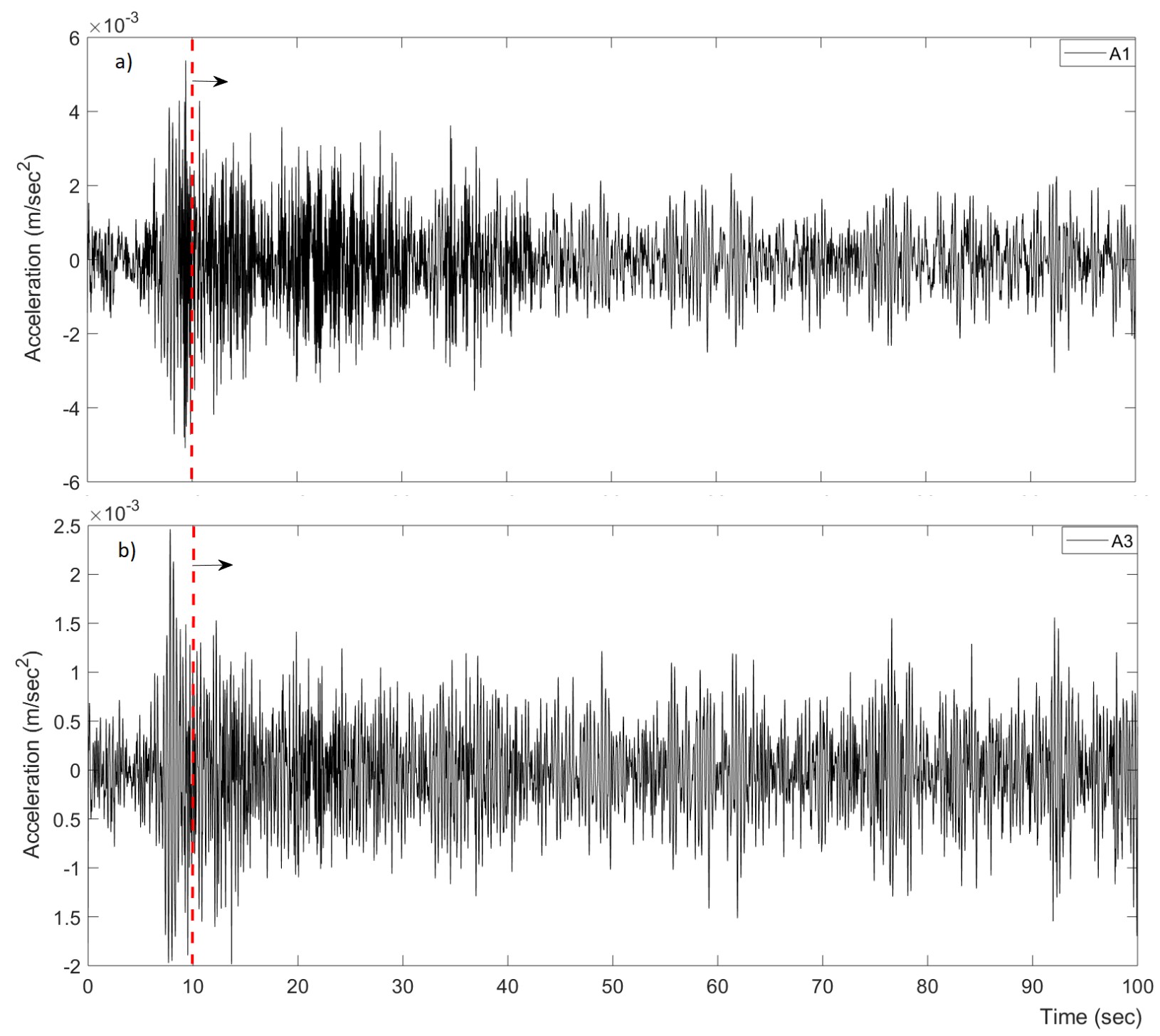

Figure 3.4. Transverse acceleration response recorded by sensors (a) 1 and (b) 3 for simulating damage response in pier damage scenario. 

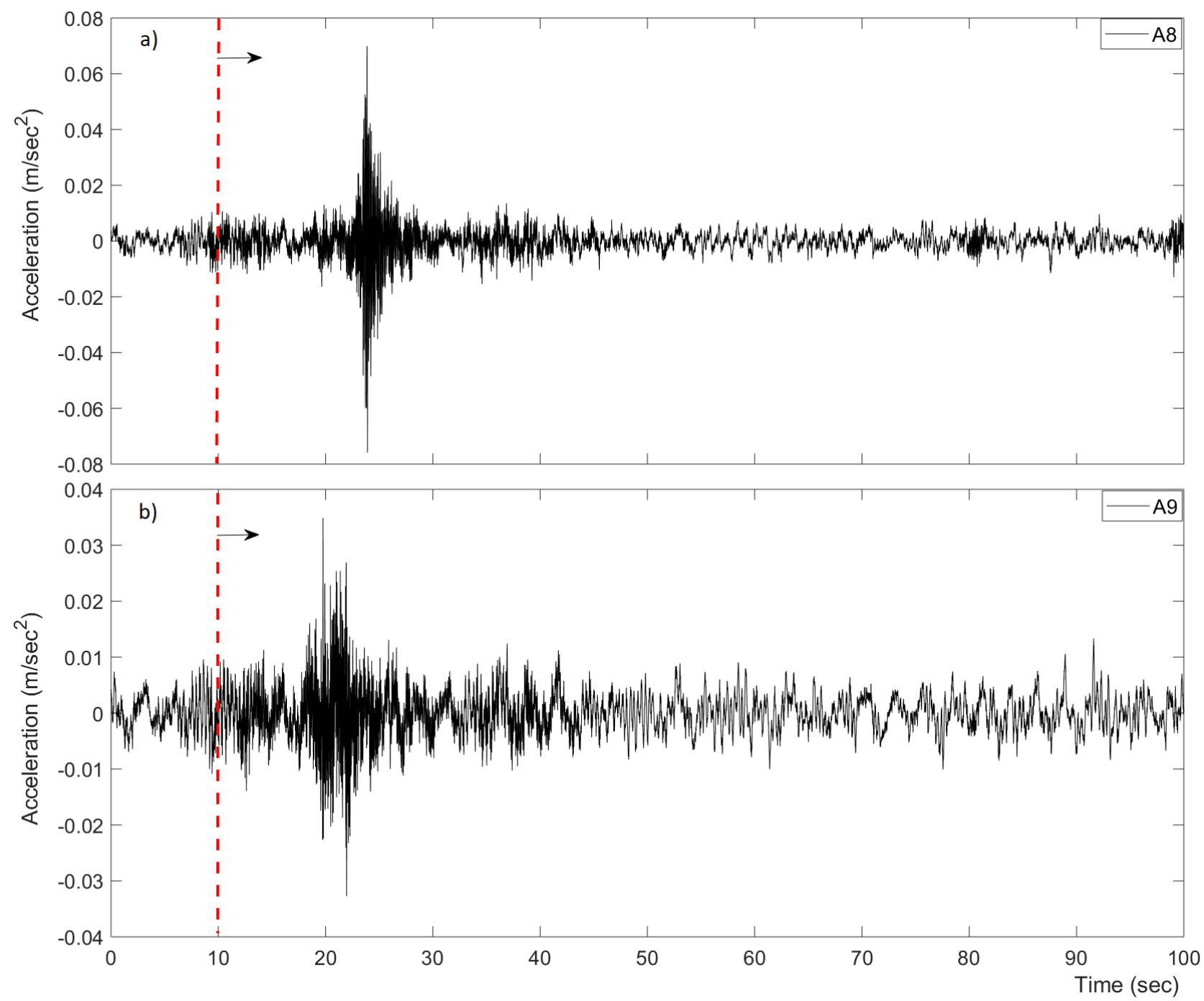

Figure 3.5. Transverse acceleration response recorded by sensors (a) 8 and (b) 9 for simulating damage response in drop-in span joint damage scenario. 

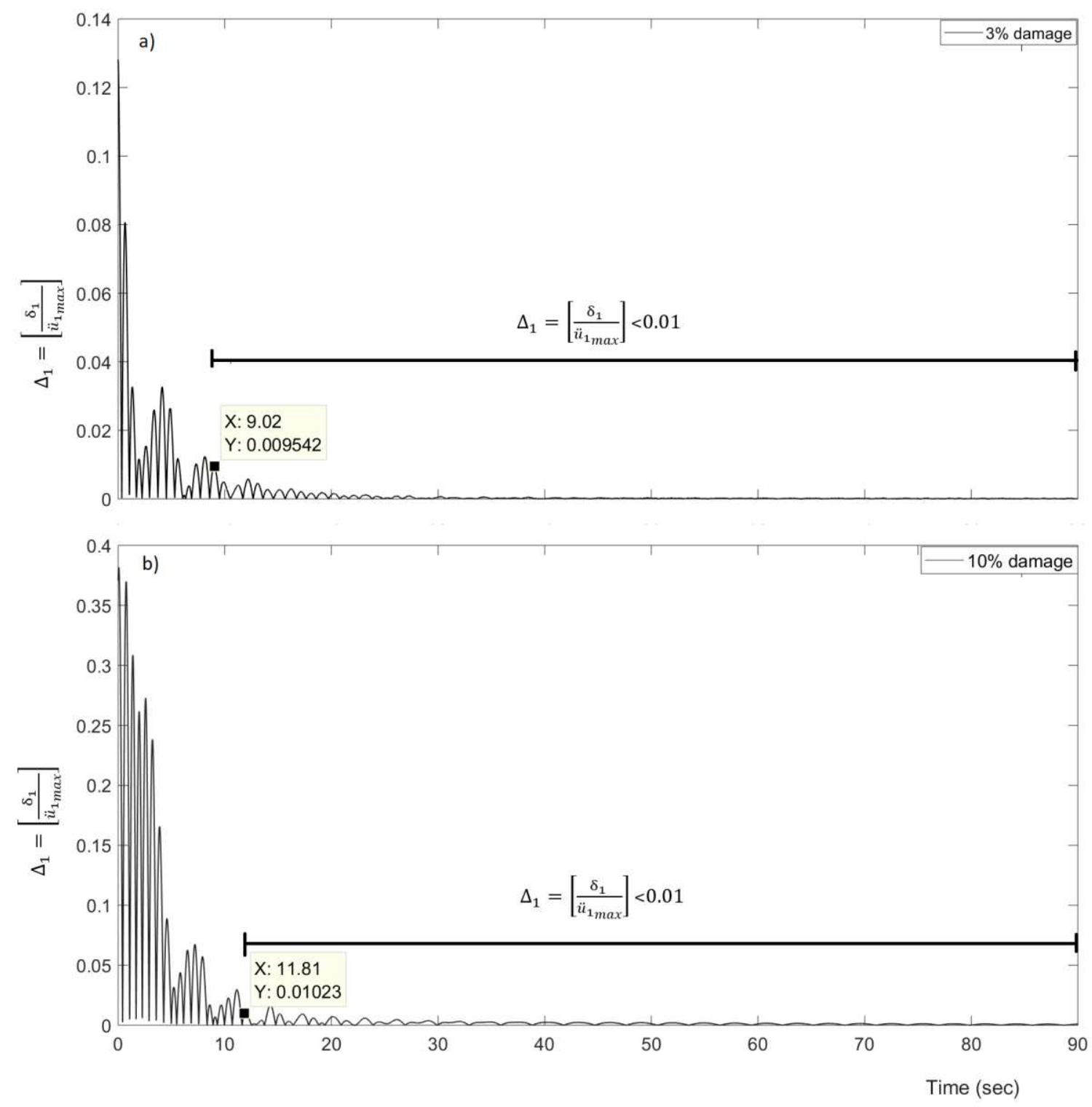

Figure 3.6. Normalized relative error in the response simulated by Pseudo load at sensor 1 for (a) $3 \%$ and (b) $10 \%$ damage in piers. 

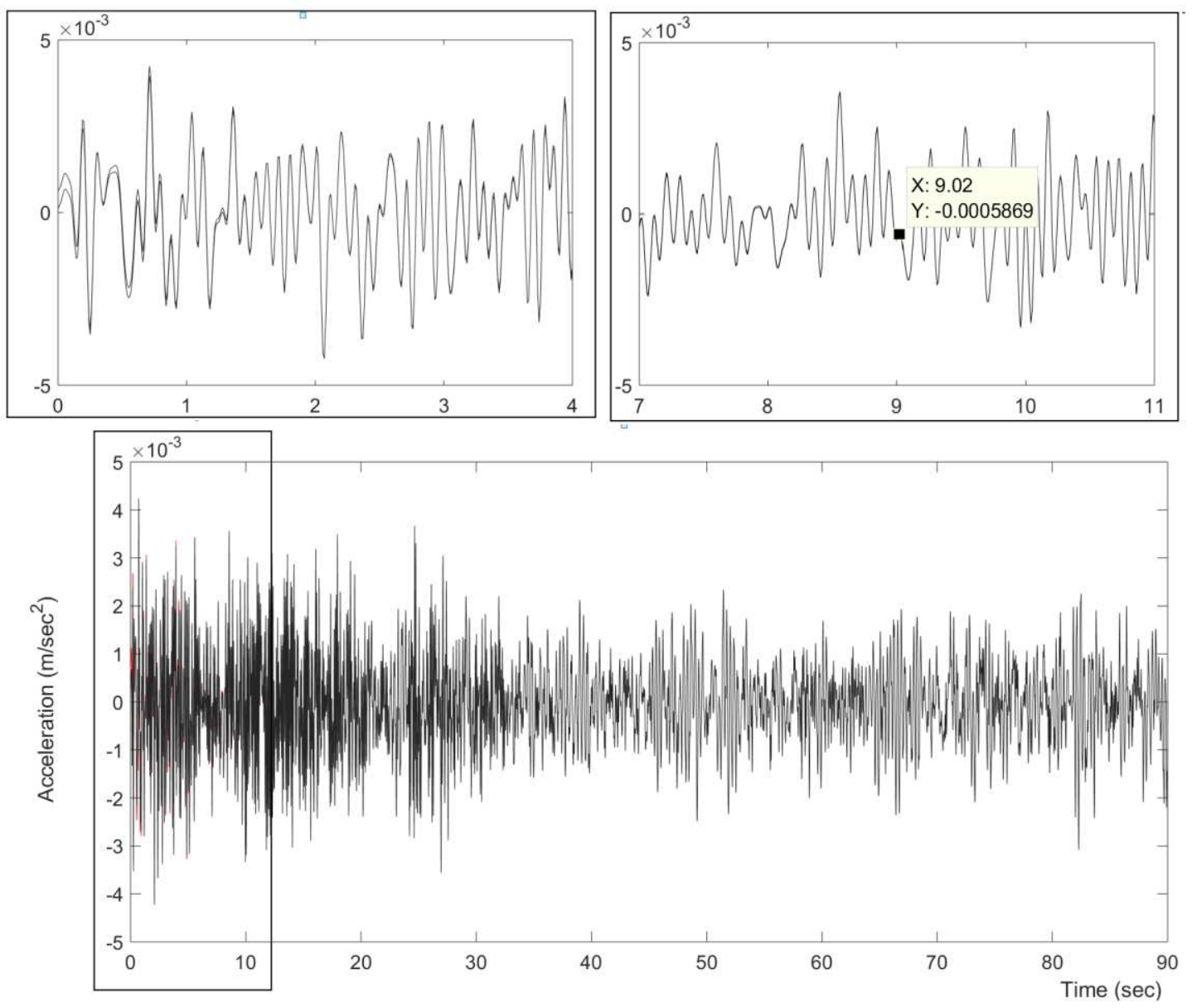

Figure 3.7. Comparison of the exact response and the response simulated by Pseudo load at sensor 1 for $3 \%$ damage in piers. 

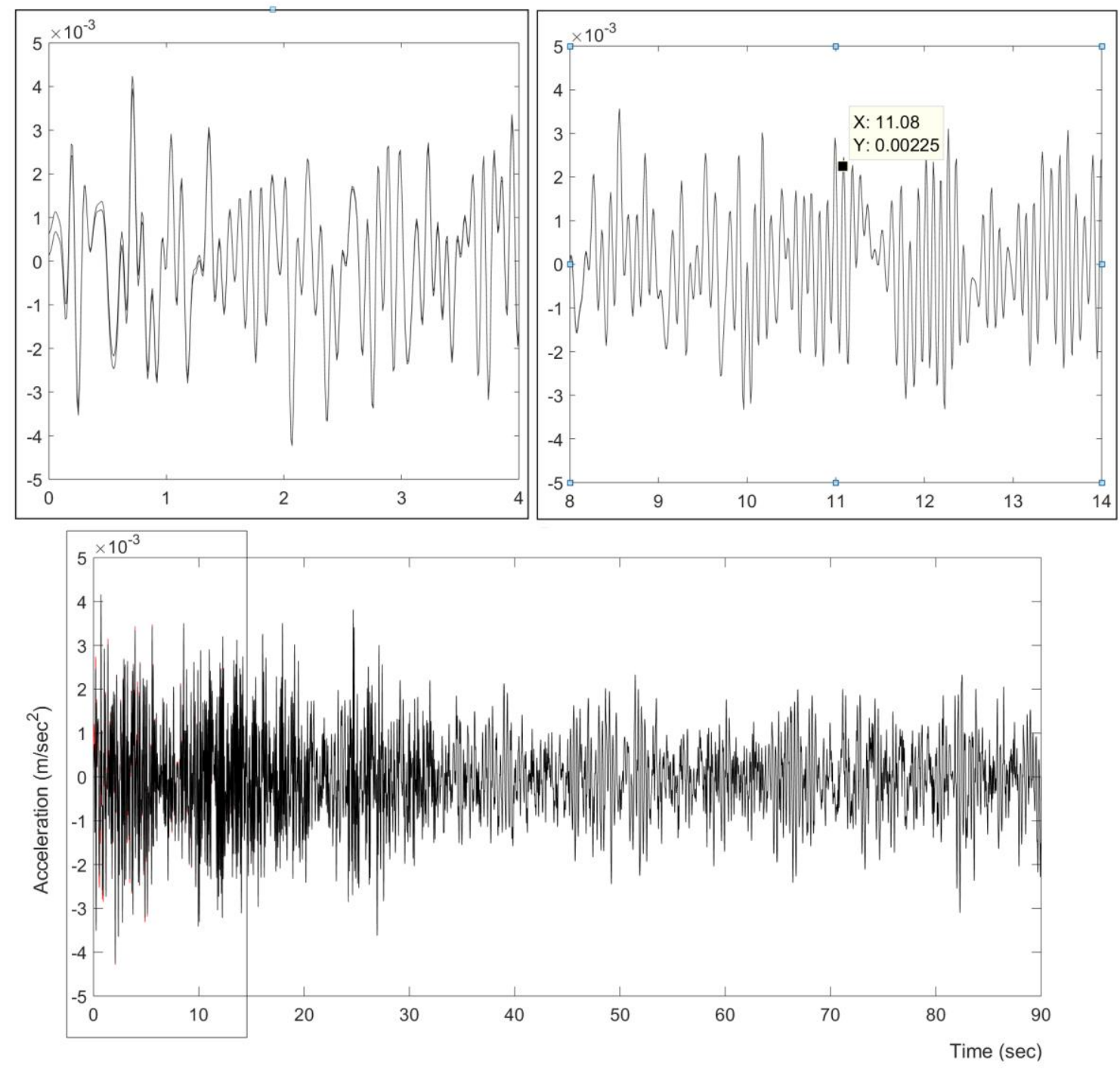

Figure 3.8. Comparison of the exact response and the response simulated by Pseudo load at sensor 1 for $10 \%$ damage in piers. 


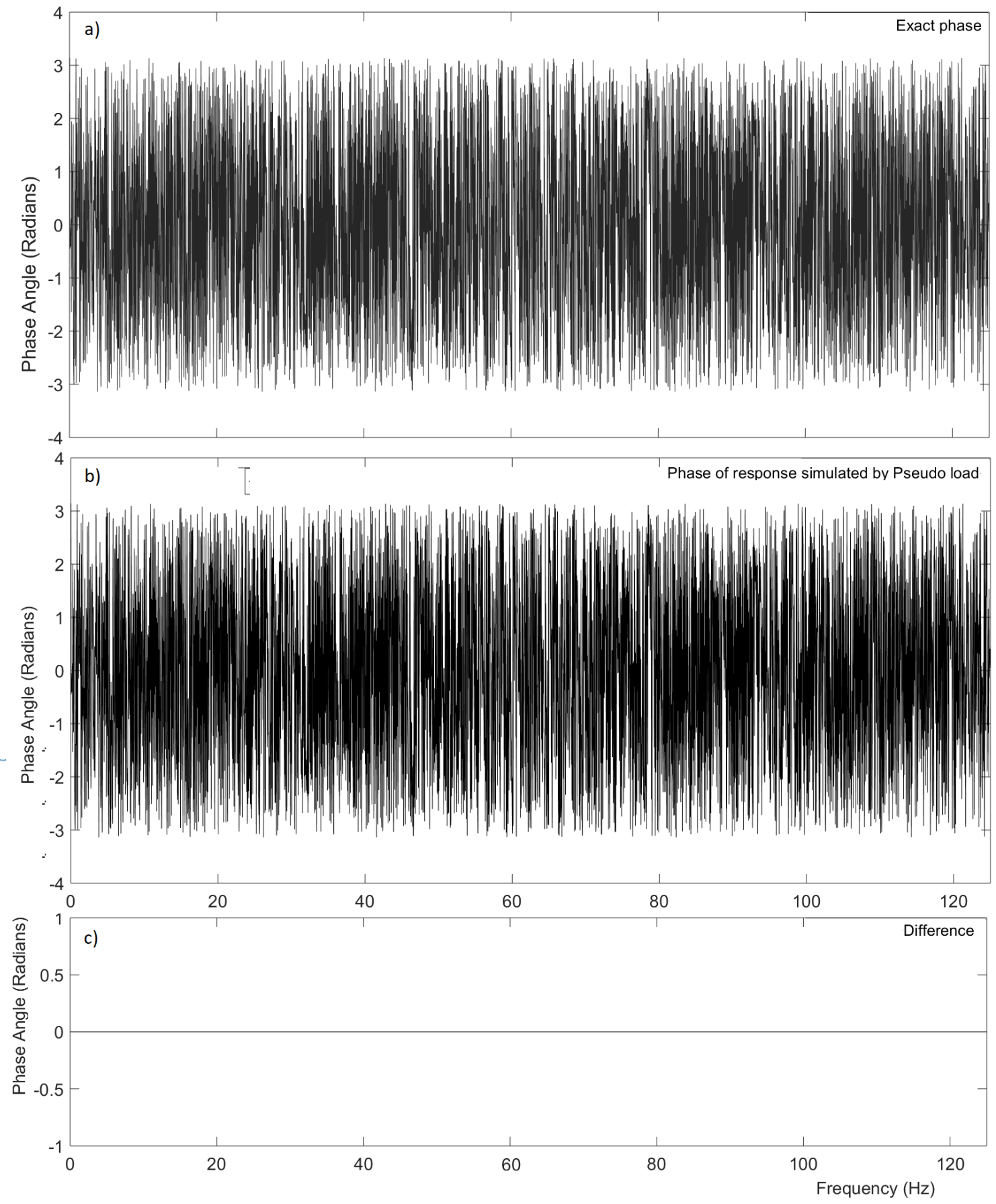

Figure 3.9. Comparison of the (a) exact phase and (b) the phase obtained from response simulated by Pseudo load at sensor 1 for $10 \%$ damage in piers that satisfies the criterion in Eq. 2.19, with (c) difference in phase results. 

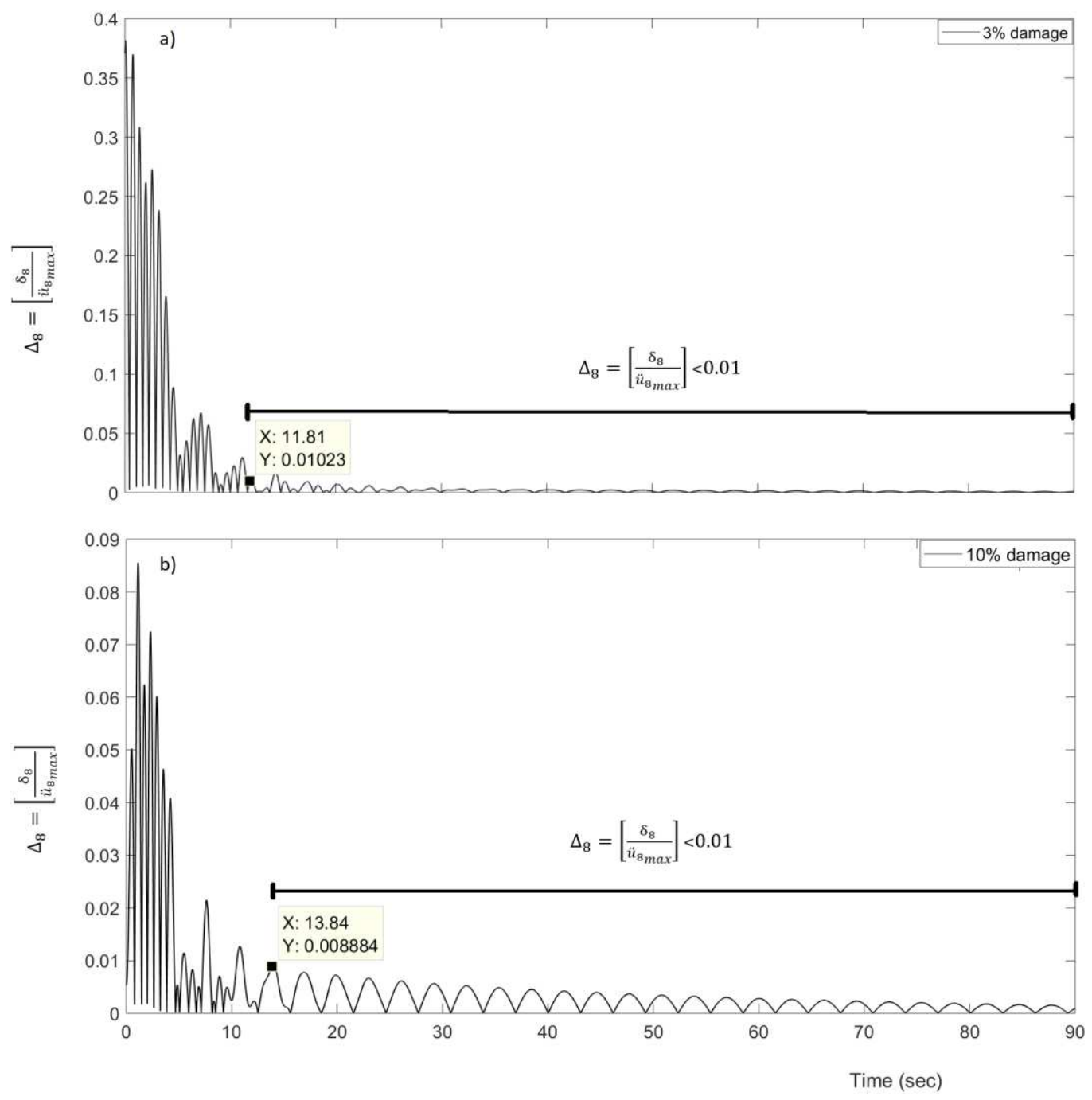

Figure 3.10. Normalized relative error in the response simulated by Pseudo load at sensor 8 for (a) 3\% and (b) 10\% damage in drop-in span joint. 

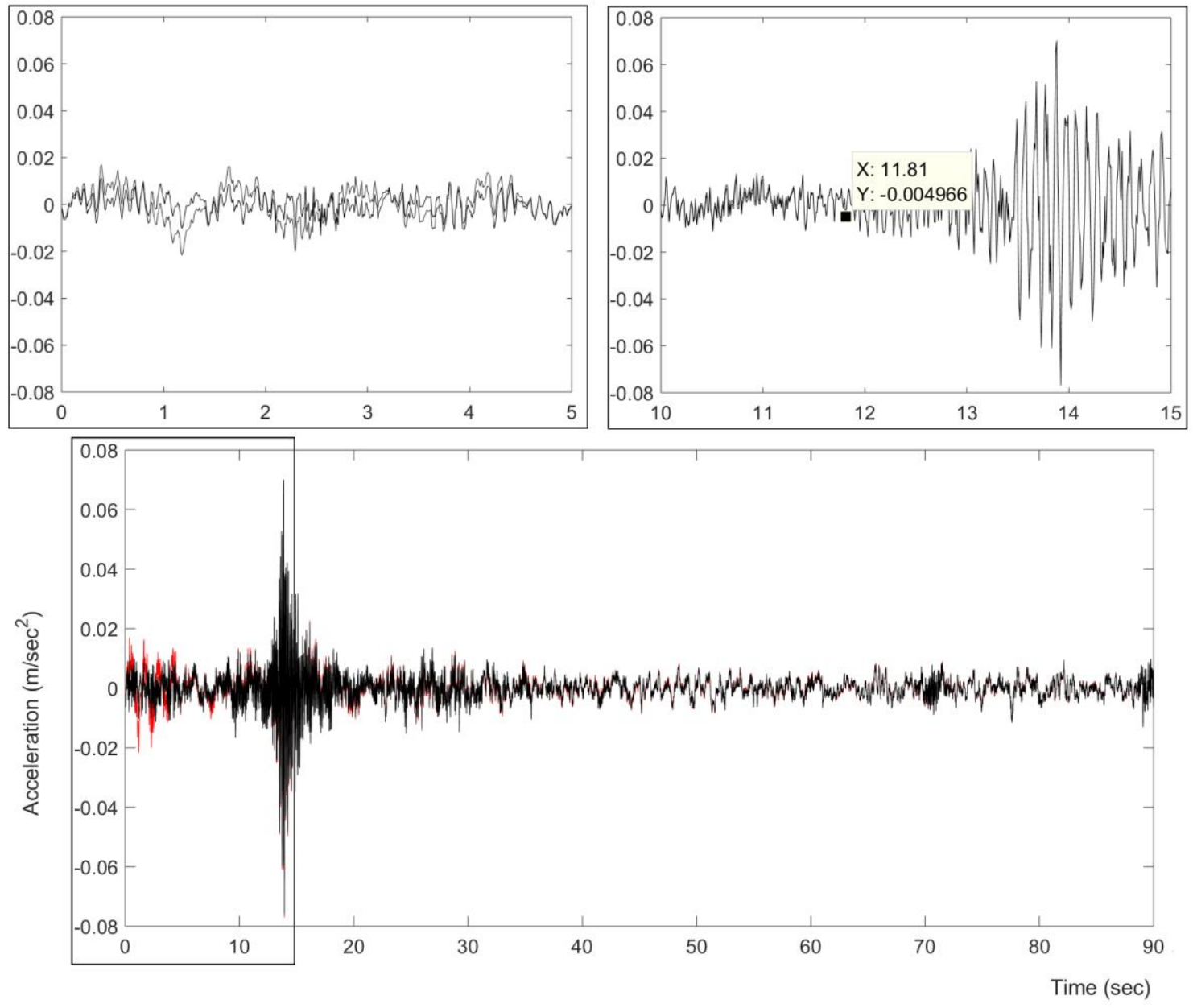

Figure 3.11. Comparison of the exact response and the response simulated by Pseudo load at sensor 8 for $3 \%$ damage in drop-in span joint. 

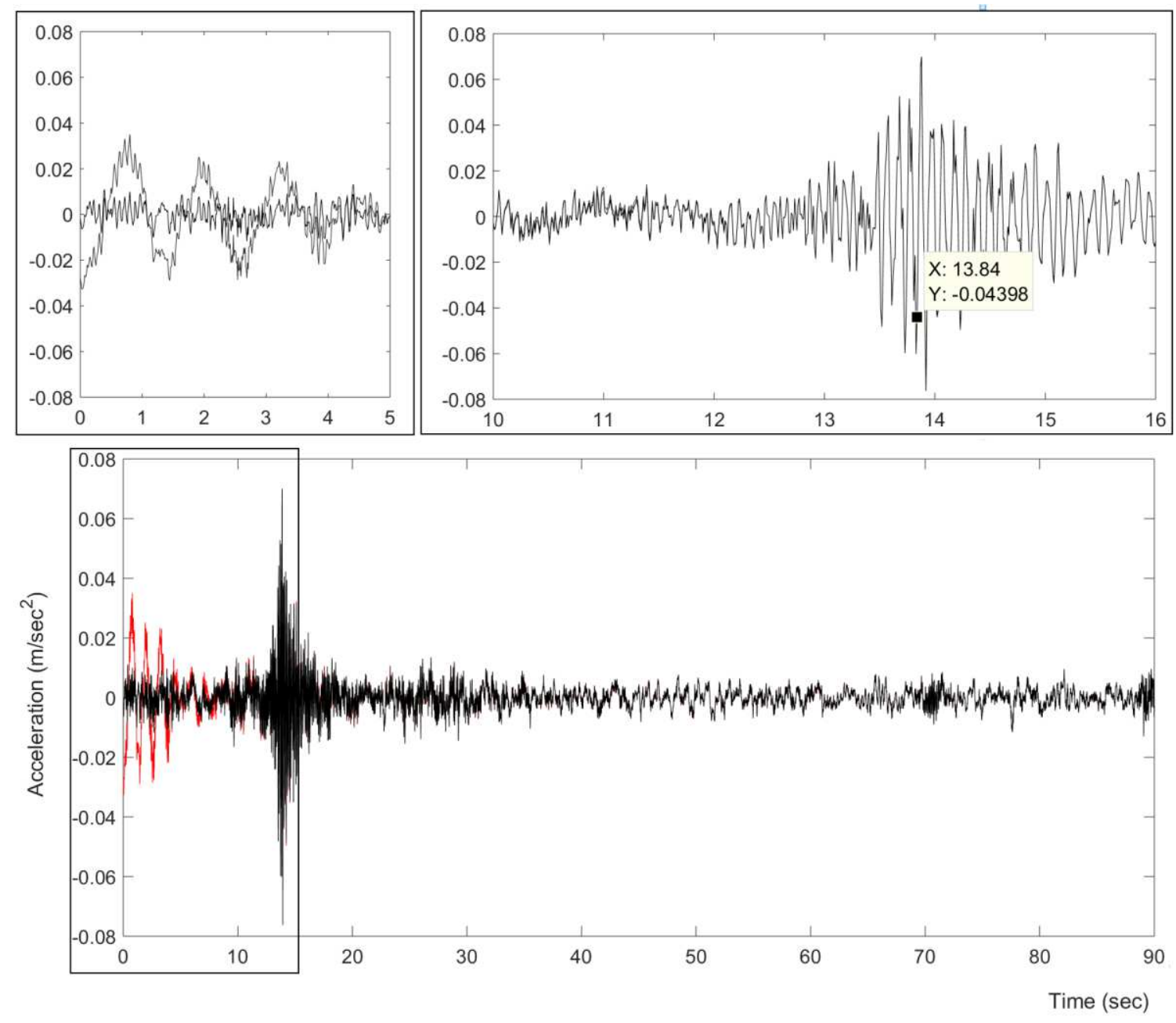

Figure 3.12. Comparison of the exact response and the response simulated by Pseudo load at sensor 8 for $10 \%$ damage in drop-in span joint. 

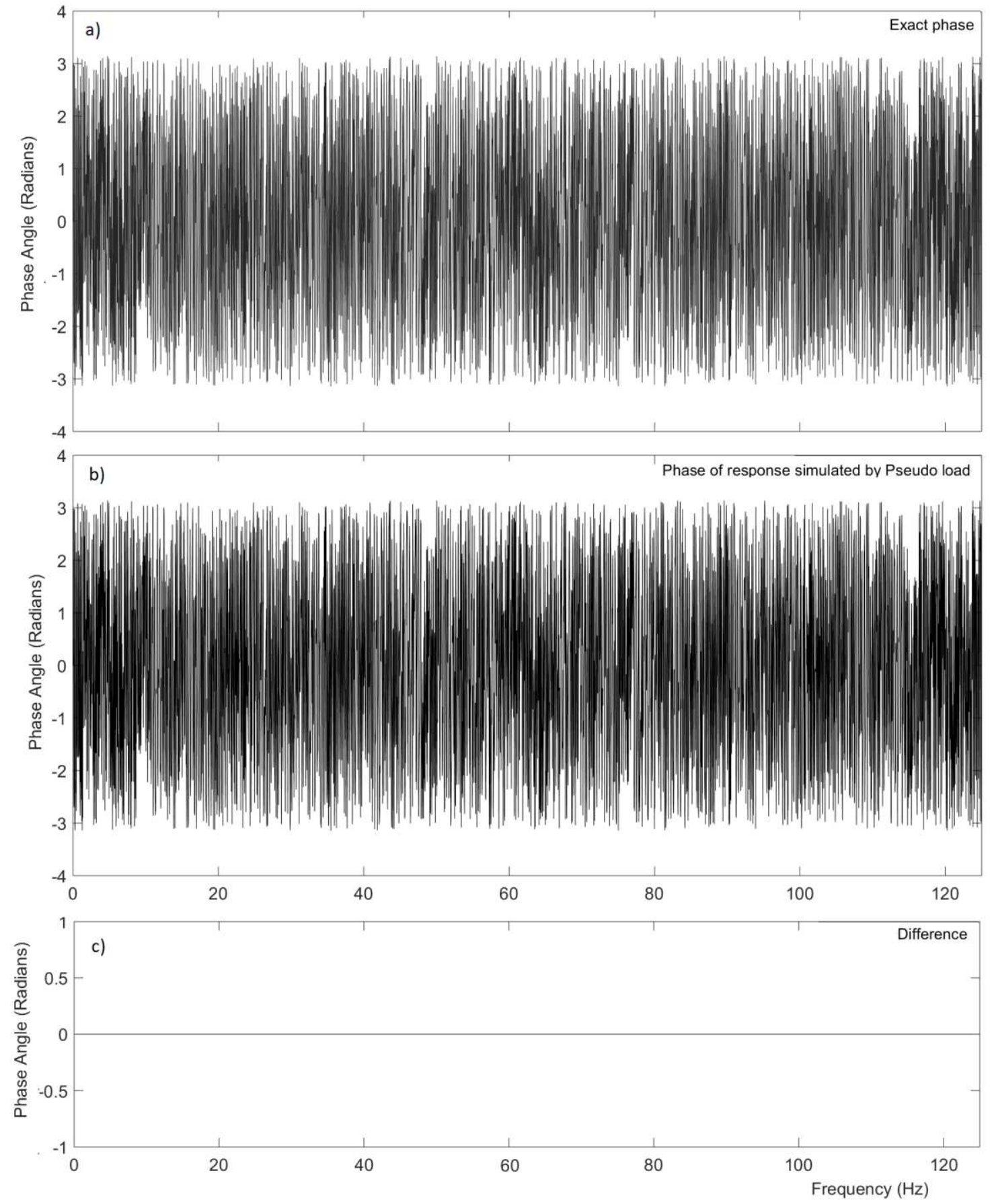

Figure 3.13. Comparison of the (a) exact phase and (b) the phase obtained from response simulated by Pseudo load at sensor 8 for 10\% damage in drop-in span joint that satisfies the criterion in Eq. 2.19, with (c) difference in phase results. 

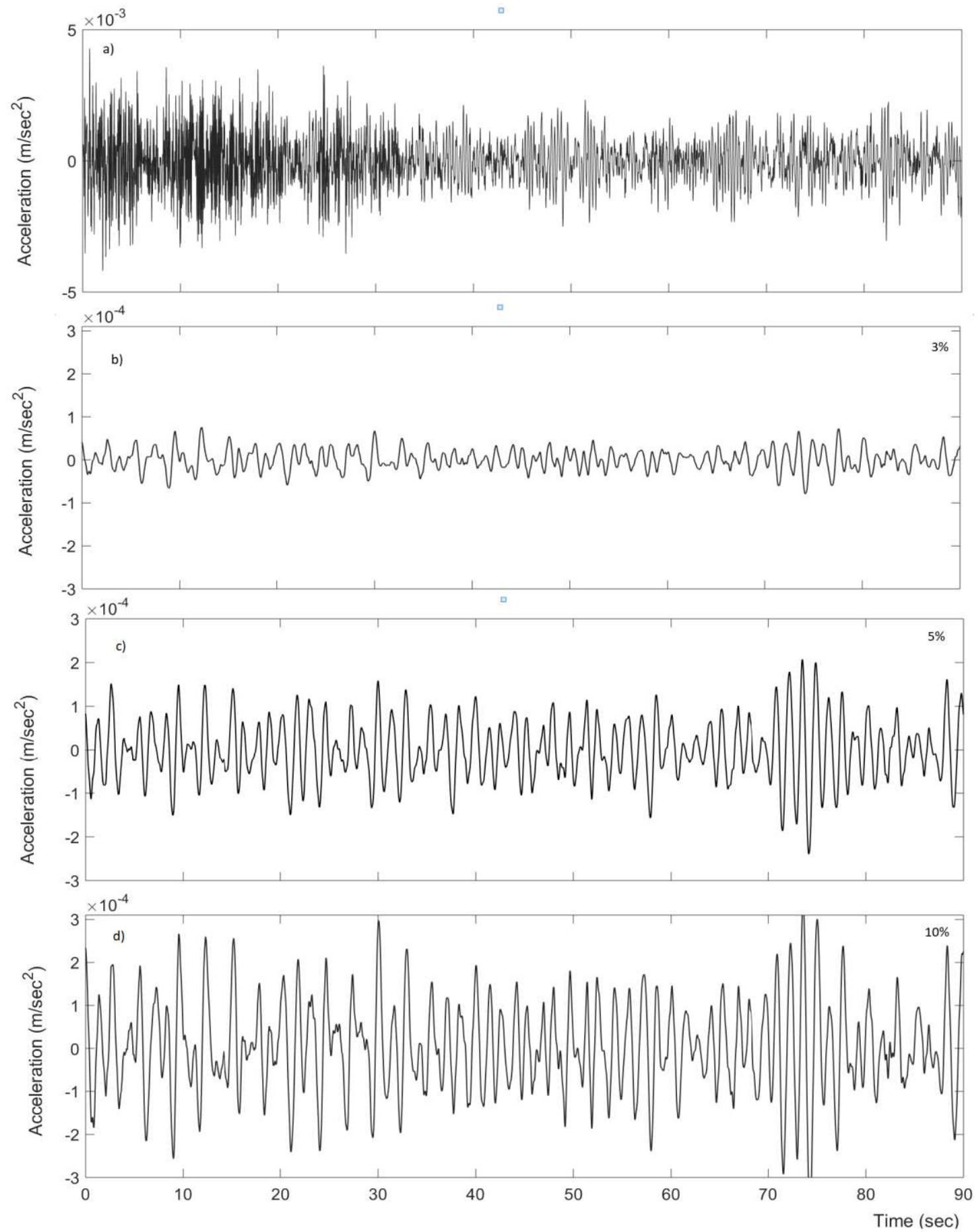

Figure 3.14. Comparison of the relative difference between the captured response and the response simulated by Pseudo load at sensor 1; (a) captured (b) difference with 3\% damage (c) difference with 5\% damage (d) difference with $10 \%$ damage. 

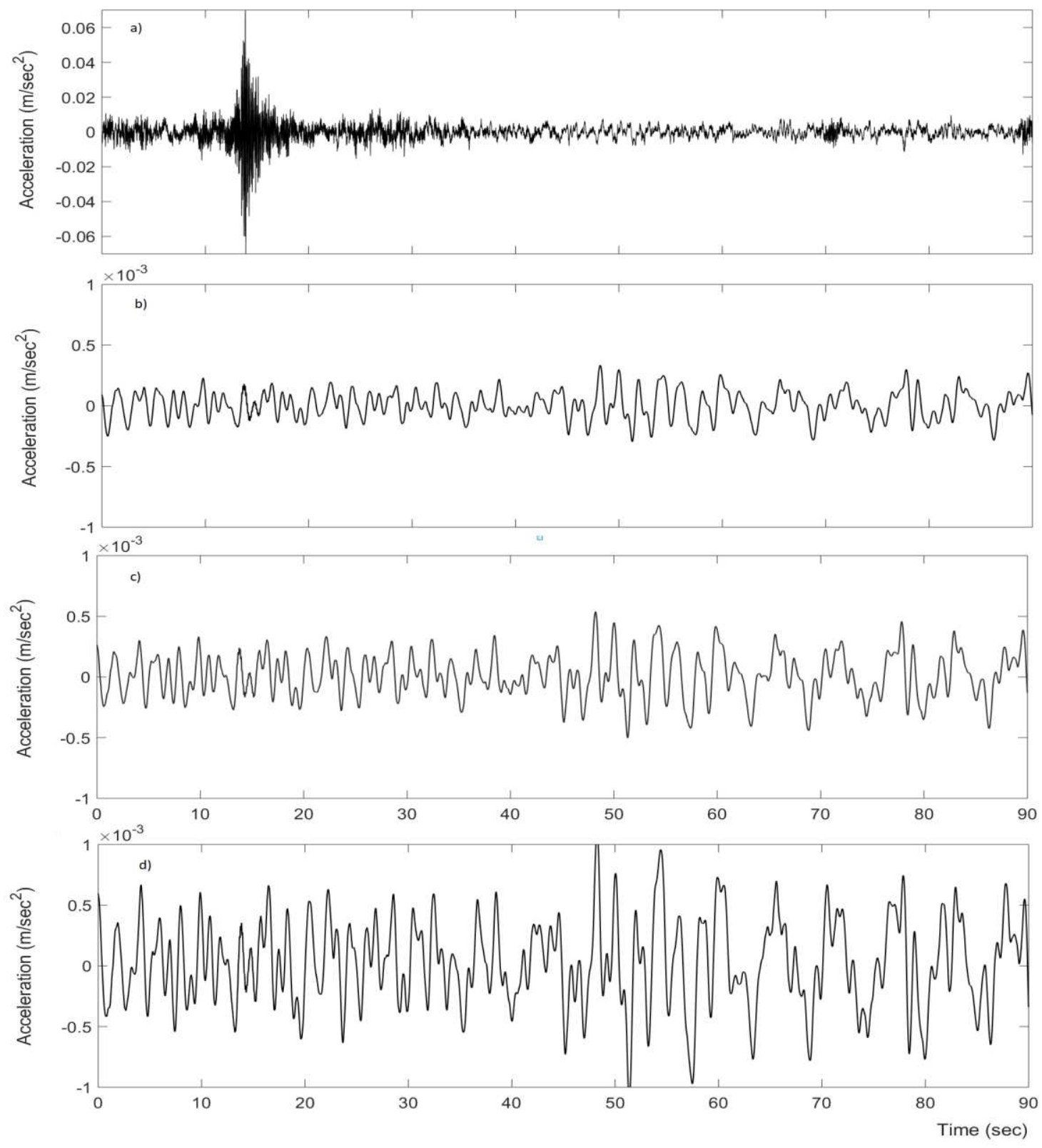

Figure 3.15. Comparison of the relative difference between the captured response and the response simulated by Pseudo load at sensor 8; (a) captured (b) difference with 3\% damage (c) difference with 5\% damage (d) difference with $10 \%$ damage. 

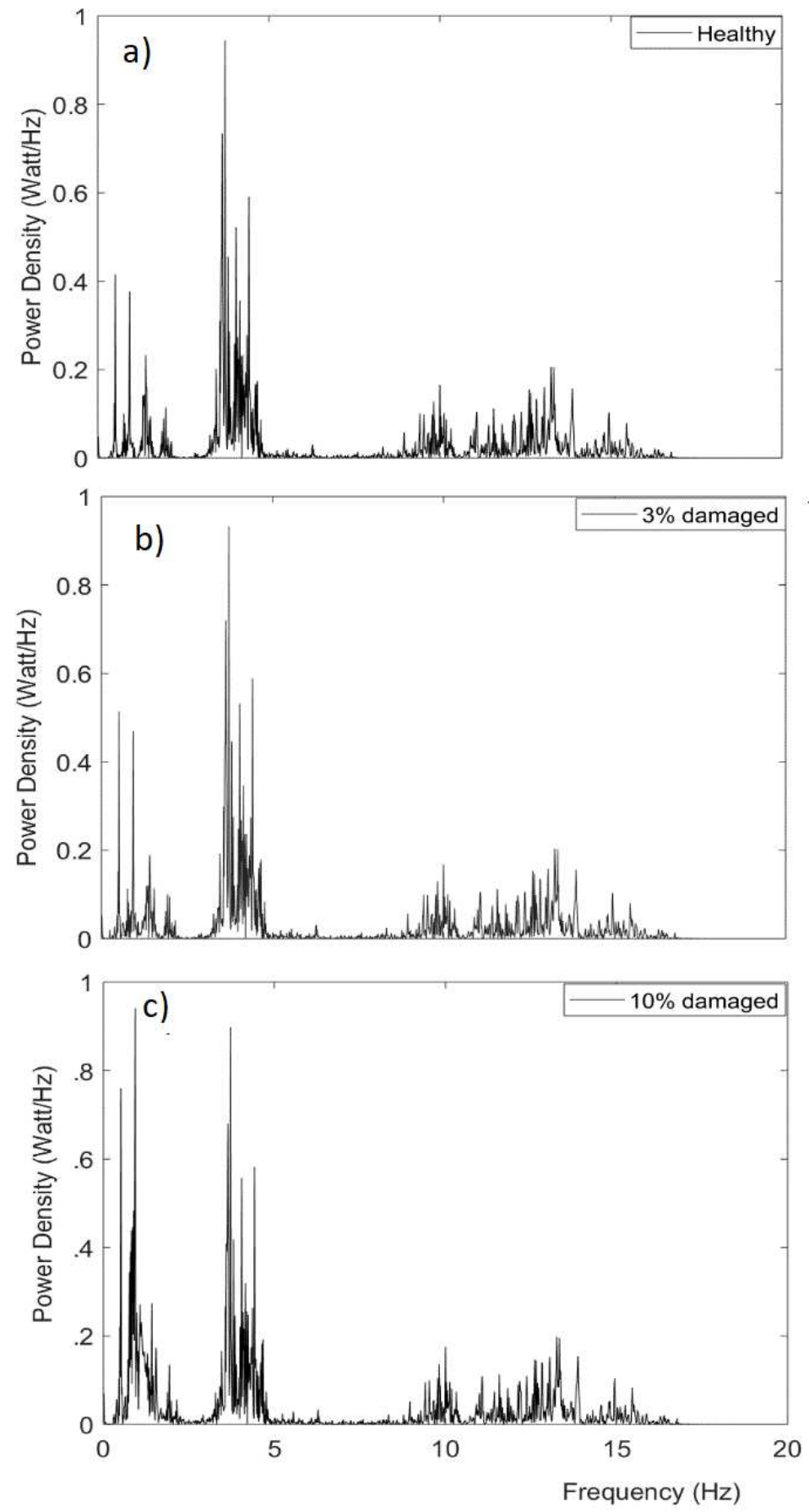

Figure 3.16. Comparison of the power spectral density of the (a) captured response and the response simulated by Pseudo load at sensor 1 for (b) $3 \%$ and (c) $10 \%$ damage in piers (up to $20 \mathrm{~Hz}$ ). 


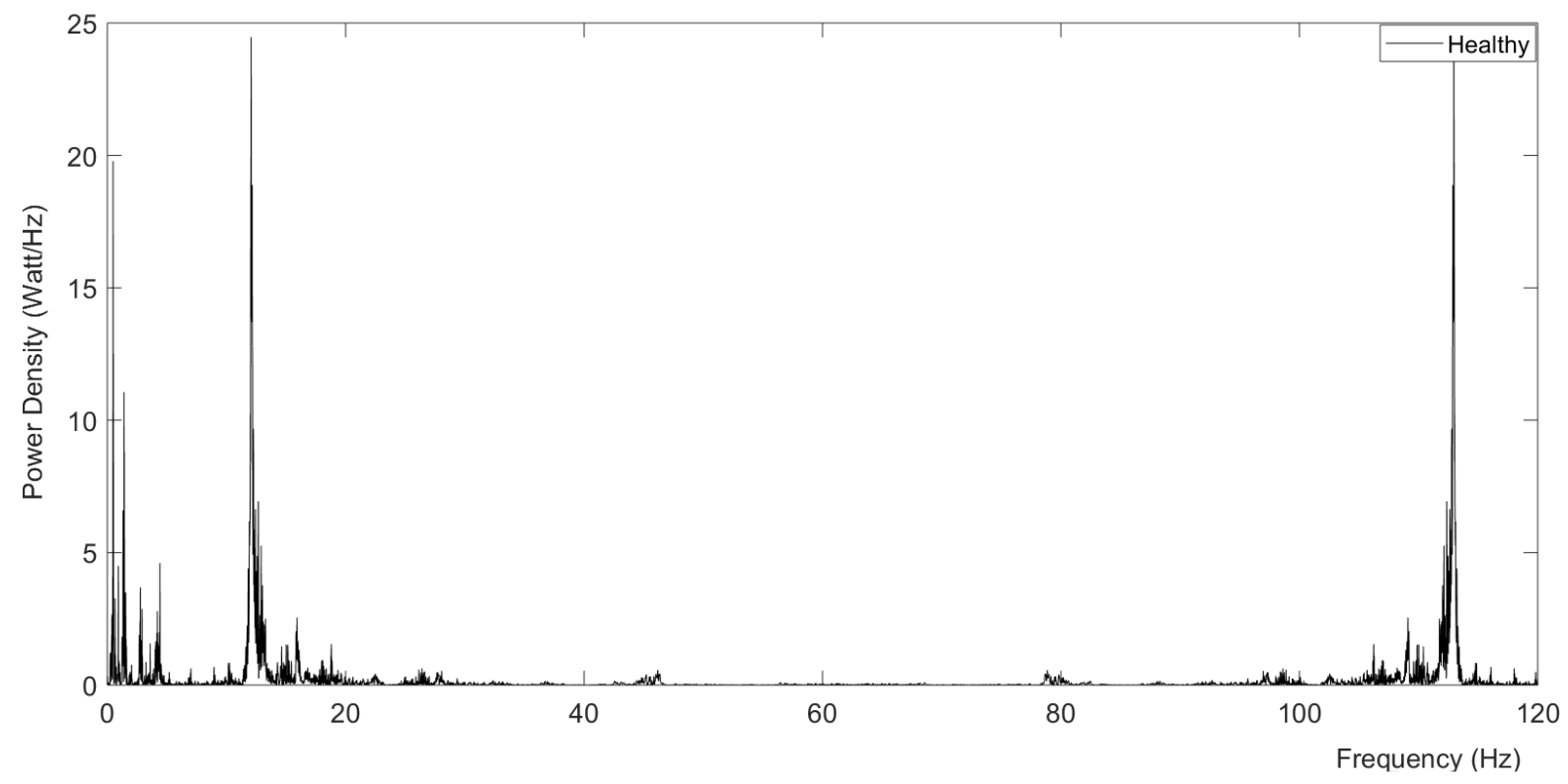

Figure 3.17. Power spectral density of the response captured by sensor 8 . 


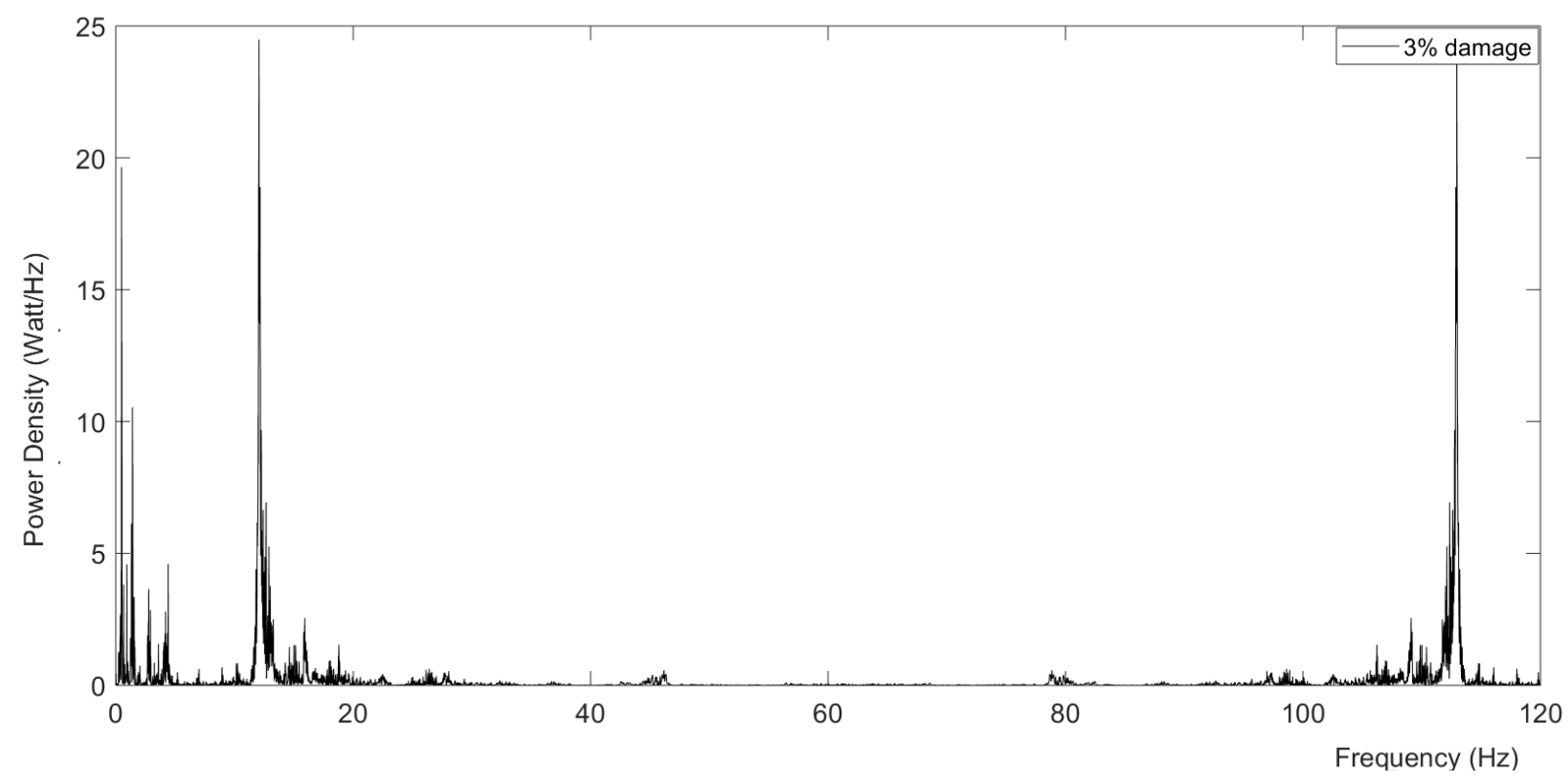

Figure 3.18. Power spectral density of the response simulated by Pseudo load at sensor 1 for 3\% damage in drop-in span joint. 


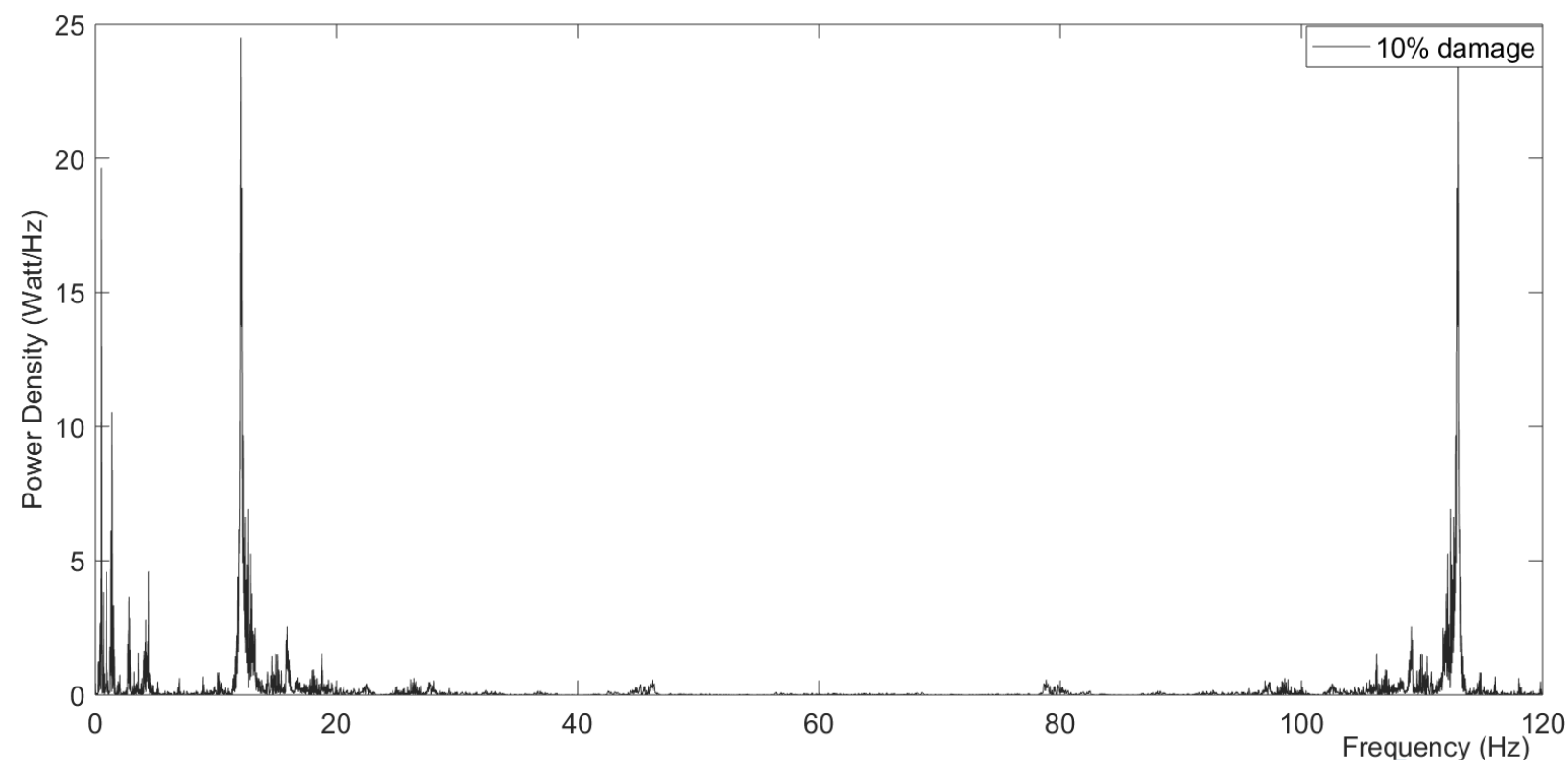

Figure 3.19. Power spectral density of the response simulated by Pseudo load at sensor 8 for $10 \%$ damage in drop-in span joint. 


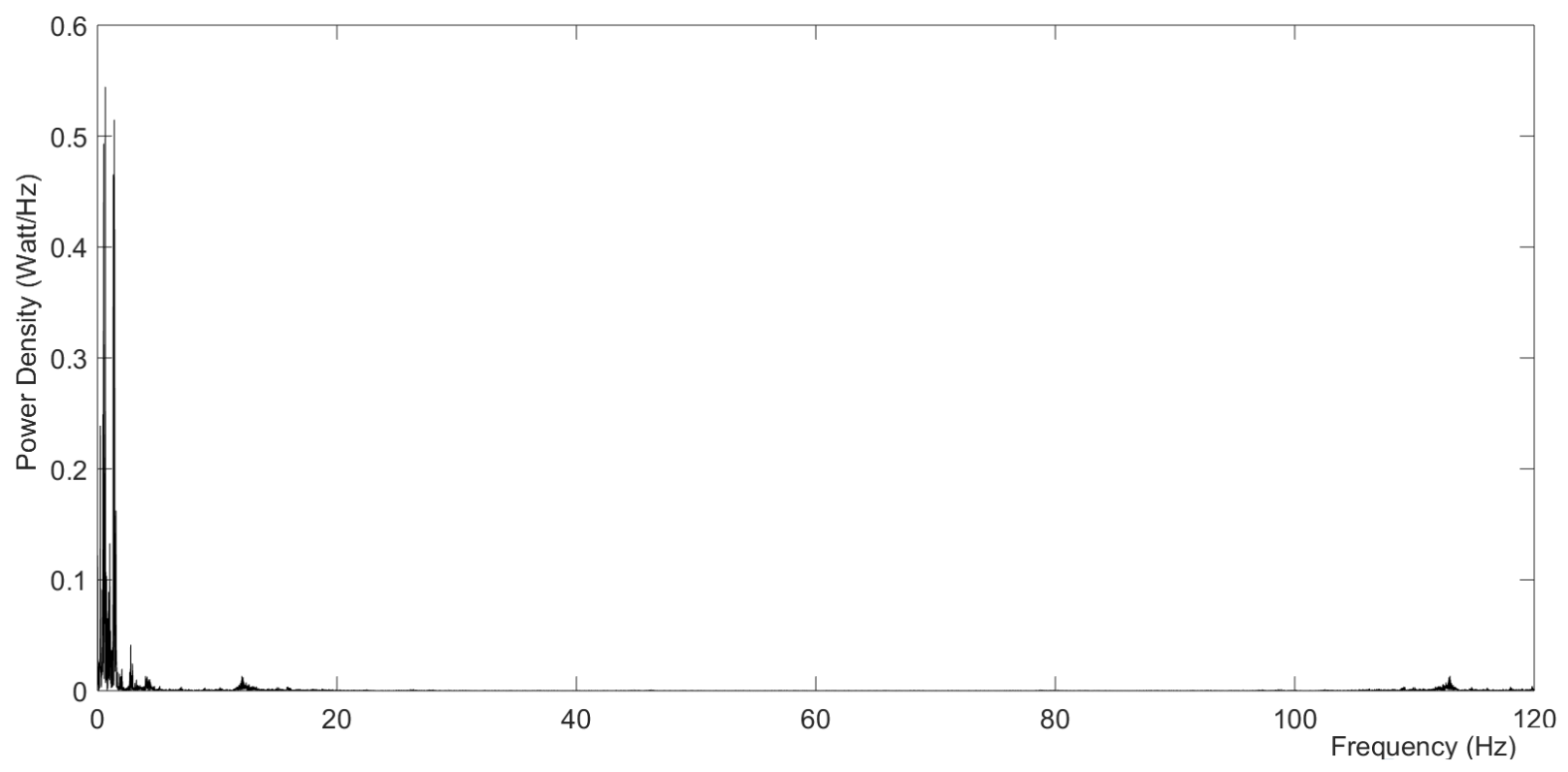

Figure 3.20. Absolute difference between the power spectral densities of the captured response and the response simulated by Pseudo load at sensor 8 for $3 \%$ damage in dropin span joint. 


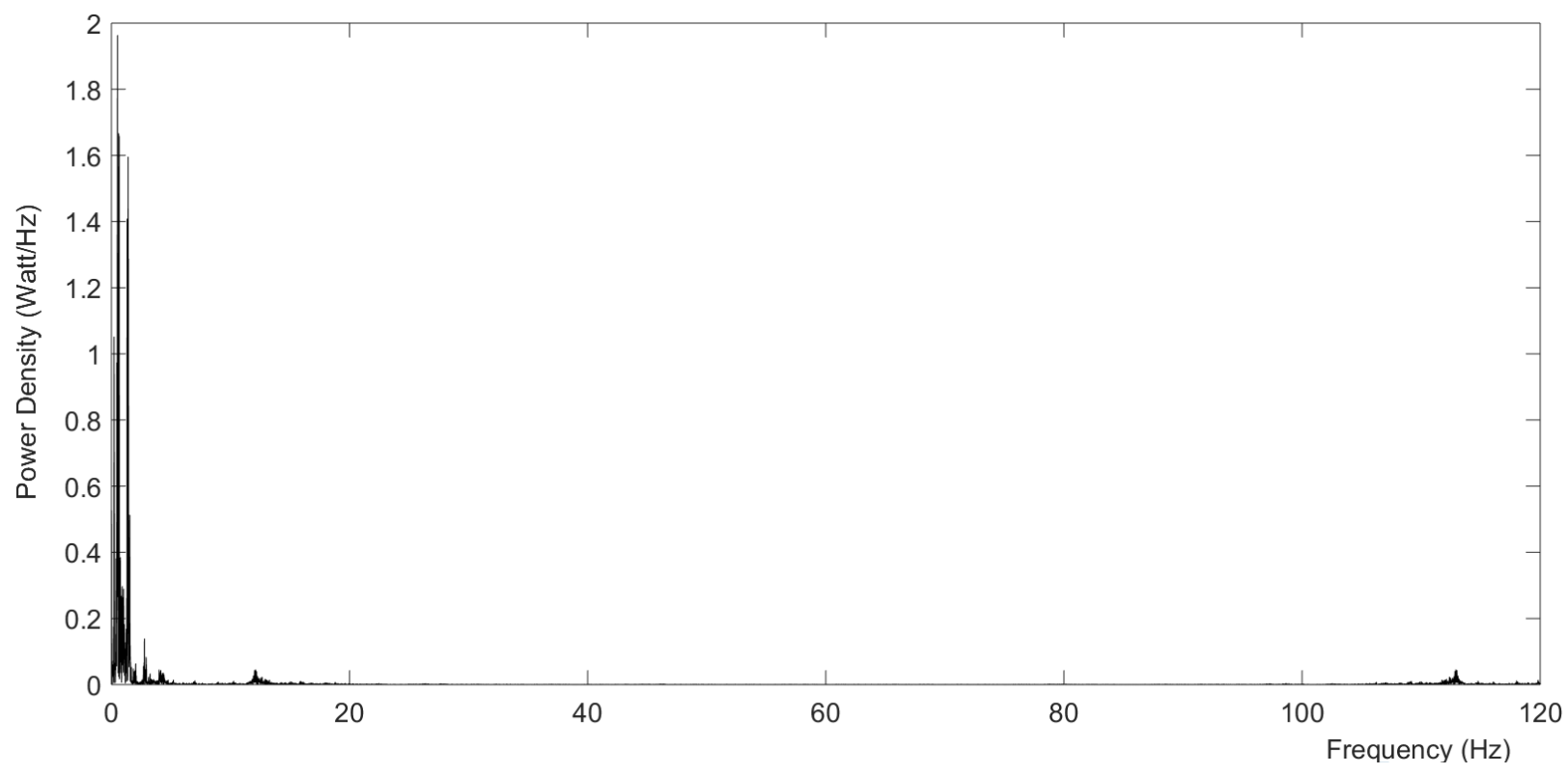

Figure 3.21. Absolute difference between the power spectral densities of the captured response and the response simulated by Pseudo load at sensor 8 for $10 \%$ damage in dropin span joint. 
Table 3.1 Initial condition for pier damage simulation

\begin{tabular}{|l|c|c|}
\hline Initial conditions & Sensor 1 & Sensor 3 \\
\hline Displacement(m) & -0.0013 & 0.0026 \\
\hline Velocity $(\mathrm{m} / \mathrm{s})$ & 0.0020 & 0.0079 \\
\hline
\end{tabular}


Table 3.2 Initial conditions for drop in damage simulation

\begin{tabular}{|l|c|c|}
\hline Initial conditions & Sensor 8 & Sensor 9 \\
\hline Displacement(m) & -0.087 & 0.073 \\
\hline Velocity $(\mathrm{m} / \mathrm{s})$ & -0.381 & 0.270 \\
\hline
\end{tabular}




\section{Chapter 4 Conclusions and Recommendations}

\subsection{Summary}

Without intervention, the performance of structures gradually deteriorates over time due to number of factors including its age from continual exposure and usage, deteriorations and cumulative damage caused by wind, traffic and earthquakes etc. The deterioration process is even more severe for structures located in hostile environment. Through the combination of the practice of structural engineering and advances in sensors, data analytics and telecommunication technologies, structural health monitoring is a relatively new field of research of developing the framework that utilizes the new technologies and advances in structural engineering, computing and sensing to improve and enhance the design and performance of built structures. The current practice of structural condition assessment is based on periodic visual inspection and structural condition surveys. It is recognized the existing practice has limitation on detecting deterioration and damage at early stage and is inadequate to provide information about the global performance and integrity of structural systems.

Over the last decades, developments in communication and sensing technology have made it possible to conduct structural assessment by means of monitoring using data acquired under their normal operations as well as during and immediately after extreme accidental or natural hazard events. In vibration based structural health monitoring (VBSHM), the vibration behaviour of the monitored structure are the primary focus in assessing its structural health. The most commonly used data in VBSHM are the vibration responses of the monitored structure under ambient conditions, and the associated metadata of the 
operational and environmental conditions at the time of the recorded responses such as the traffic and weather conditions of temperature, humidity and wind etc. In recent years, research of VBSHM have led to considerable advances and improvements in the level of details and accuracy and the broadening of scope in structural risk and condition assessment applications. In comparison to the current practice and technology of sending out an inspection team of engineers to collect data every time when data are needed, VBSHM can collect relatively inexpensively without service disruptions vast amount of data, or big data, continuously on the structural health for analysis and assessment. Combining all these advantages and potential, it can be recognized that VBSHM is ideally suitable for implementation in long-term continuous structural health monitoring of large complex structures.

It is recognized that VBSHM faces some limitations in practical applications. One such limitation is the influence of uncertainties on the vibration responses and characteristics of the monitored structure that comes from variabilities or noises in the operational and environmental conditions of the structure, such as the boundary conditions, material properties, load actions, traffic, temperature and wind condition of wind speed and direction etc. The variabilities in the vibration responses due to these background noise and uncertainties may either obscure or give false impression of damage. The sources of noise and uncertainties associated with extracted vibration characteristics of monitored structures and their implications on assessment of structural health have been recognized and recorded in the literature. However, the current state-of-art in VBSHM is still not able to provide effective solutions to overcome the challenge of reliably distinguish between 
changes in vibration behaviour that are caused by actual damage from those due to noise and uncertainties of its operation or environment.

In light of the information provided by literature, in context to VBSHM, researchers have developed algorithms that attempt to overcome the challenges posed by variabilities in the operational and environmental conditions, in detecting damages in real world structures. Some of these developed algorithms are tested on data which do not consider the effect of field noise and uncertainties. Some developed algorithms are tested for their effectiveness in identifying changes in structural response parameters which are influenced by selected field variables like temperature, wind and traffic etc. In real world problems, civil engineering structures can have the noise and uncertainty effects for wide range of sources which may be even unknown or highly impractical to quantify. Therefore, the algorithms developed and tested on such statistical data that incorporates only selected sources of uncertainties have limited practical applications. Moreover, it is difficult or highly impractical to develop comprehensive statistical models that can quantify all the sources of noise and uncertainties and their effect on the vibration bahaviour of monitored structures in the field. Also, the statistical models developed for one monitored structure cannot be readily applied to other structures since the noise and uncertainty effects in different operational and environmental condition may be different. Therefore, to overcome the limitations inherent in the statistical models an alternate approach is proposed in this thesis that not only can be used to simulate the damage response of monitored structures with realistic noise and uncertainties in the field but has the universal applicability to all monitored structures. 
In the proposed Pseudo load methodology actual field monitoring data are used as the basis for simulating the damage structure response data. Since the field monitoring data preserve all the noise and uncertainties of the structural response behaviour and environment conditions, as such they are the exact representation of the field noise and uncertainties. The proposed methodology duplicates the noise and uncertainties present in the field captured responses and applies them to the simulated damage response data of the finite element model of the monitored structure. The damage response generated therefore has the damage characteristics of the monitored structure and the realistic field noise and uncertainties. Such a data can be used to examine the effectiveness of existing vibration based algorithms in detecting damages in real structures, as well as for developing more advanced vibration based structural condition assessment tools.

\subsection{Contributions of Research}

The main contribution of this research is that it recognizes one of the most difficult challenges faced in the field of VBSHM, and consequently this research develops a simple and yet effective method to overcome those challenges. The Pseudo load method developed in this research can effectively and reliably simulate the damage responses of structures with field noise and uncertainties as demonstrated through numerical examples. This work is different from others since it considers the realistic noise and uncertainties from field operations and environmental conditions and is applicable to all monitoring structures. In

this research, it is shown without ambiguity that in presence of realistic noise and uncertainties the differences between the captured response and the simulated damage 
response are due to damage alone. This has never been demonstrated in previous VBSHM research.

In this method, the development of statistical and noise models is not required for simulating the damage response which is a significant advantage in terms of time and computing effort and resource requirements. The procedure is based on structural dynamics theory that is easy to understand and apply in practice. The results of this research can be used to investigate the effectiveness of statistical models and other damage detection algorithms in detecting damages which may be masked by noise and uncertainties.

\subsection{Conclusions and Future Works}

The concept of Pseudo load and its use in simulating the damage response of structures with realistic noise and uncertainties is discussed in this thesis. Numerical examples are provided that demonstrates the effectiveness of this method. The method is first applied and tested on theoretical problems with noise and uncertainties from the field as well as on random data. The results of application of the Pseudo load method show its effectiveness in retaining noise and uncertainties in the simulated damage responses, when compared with exact damage response results. The new method is then applied to simulate the damage responses of the Confederation Bridge by using the field captured vibration responses from the Confederation Bridge monitoring system.

For future work, the response simulated by Pseudo load method can be used as an evaluation tool to estimate the effectiveness of the existing damage detection methodologies and algorithms in detecting damages when they are masked realistic by noise and uncertainties as present in the field. Furthermore, advanced damage detection 
tools can be developed for practical problems that can reliably detect damages at reasonably early stages under the inevitable noise and uncertainties in the operational and environmental conditions. It is only with the ability to differentiate the correct influence of noise and uncertainties in the vibration response from that caused by damages that significant advances can be made in VBSHM and the true/correct structural conditions can be assessed so that remedial measures can be taken in time. This will not only help in minimizing maintenance and repair cost but will prevent any chances of potential failure and prolong the performance of structure. 


\section{References}

1. Alampalli, S. (1998), "Influence of In-service Environment on Modal Parameters", Proceedings of the 16th International Modal Analysis Conference (In Proceeding of SPIE), Vol. 3243, pp. 111-116.

2. Cawley, P., and Adams, R.D. (1979), "The location of Defects in Structures from Measurements of Natural Frequencies.” Journal of Strain Analysis, Vol. 4, pp. 49-57.

3. Desjardins, S. L. (2004), "Real time Computer Platform for Vibration Based Structural Health Monitoring of the Confederation Bridge, MASc. Thesis, Department of Civil and Environmental Engineering, Carleton University, Ottawa, Canada.

4. Desjardins, S.L., Londoño, N. A., Lau, D.T. (2006), "Real-Time Processing, Analysis and Visualization for Structural Monitoring of the Confederation Bridge", Journal of Advanced Structural Engineering, Vol. 9(1), pp. 141-157.

5. Farrar, C.R., Doebling, S.W., Starser, E.G., Cornwell, P.J. (1997), "Variability of Modal Parameters Measured on the Alamosa Canyon Bridge",15th International Conference on Modal Analysis, Vol. 1, pp. 257-263.

6. Figueiredo, E., Park, G., Farrar, C.R., Worden K., Figueiras, J. (2010), "Machine Learning Algorithms for Damage Detection under Operational and Environmental Variability", Journal of Structural Health Monitoring, Vol. 10(6), pp. 559-572.

7. Humar, J., Bagchi A., and Xu, H. (2006), "Performance of Vibration Based Techniques for the Identification of Structural Damage”, Journal of Structural Health Monitoring, Vol. 5(3), pp. 215-241. 
8. Hu, W. H., Moutinho, C., Magalhães, F., Caetano, E., and Cunha, L. (2009), “Analysis and Extraction of Temperature Effects on Natural Frequencies of a Footbridge Based on Continuous Dynamic Monitoring”, IOMAC-3rd International Operational Modal Analysis Conference, Ancona, Italy, pp. 55-62.

9. He, L., Lian, J., Ma, J. (2014), "Intelligent Damage Identification Method for Large Structures Based on Strain Modal Parameters”, Journal of Vibration and Control, Vol. 20(12), pp. 1783-1795.

10. Kim, C. Y., Jung, D. S., Kim, N. S. and Yoon, J. G. (2001), "Effect of Vehicle Mass on Measured Dynamic Characteristics of Bridge from Traffic-Induced Vibration Test", Proceedings of the 19th International Modal Analysis Conference, Society for Experimental Mechanics, Bethel, Connecticut, USA, pp. 1106-1111.

11. Kullaa, J. (2003), “Elimination of Environmental Influences from Damage Sensitive Features in a Structural Health Monitoring System”, Structural Health Monitoring The Demands and Challenges, edited by Fu-Kuo Chang, CRC Press, pp. 742-749.

12. Kullaa, J. (2004), "Structural Health Monitoring under a Variable Environmental or Operational Conditions”, In Proceeding of Second European Workshop on Structural Health Monitoring, Munich, Germany, pp. 1262-1269.

13. Kullaa, J. (2010), "Vibration Based Structural Health Monitoring Under Variable Conditions Environmental or Operational Conditions", New Trends in Vibration Based Structural Health Monitoring, Vol. 520, pp. 107-181.

14. Kullaa, J. (2014), "Structural Health Monitoring Under Non-Linear Environmental or Operational Influences", Journal of Shock and Vibration, Vol. 2014, pp. 1-9. 
15. Londoño, N. A., Lau, D. T. (2003), "Variability of Dynamic Properties from Confederation Bridge Monitoring Data", Proceedings of the First International Conference on Structural Health Monitoring and Intelligent Infrastructure, Tokyo, Japan, Vol. 1, pp. 543-550.

16. Londoño, N. A. (2004), "Use of Vibration Data for Structural Health Monitoring of Bridges”, Ph.D. Thesis, Department of Civil and Environmental Engineering, Carleton University, Ottawa, Canada.

17. Londoño, N. A., Lau, D.T., Rahman, M. (2013), “Characteristics of Dynamic Monitoring Data and Observed Behaviour of the Confederation Bridge due to Operational Load Variations”, Canadian Journal of Civil Engineering, Vol. 40(5), pp. 393-409.

18. Li, Z., Feng, M.Q., Luo, L., Feng, D., Xu, X. (2018), “Statistical Analysis of Modal Parameters of a Suspension Bridge based on Bayesian Spectral Density Approach and SHM Data”, Journal of Mechanical System and Signal Processing, Vol. 98, pp. 352367.

19. Moorthy, S. and C.W. Roeder (1992), “Temperature Dependent Bridge Movements”, ASCE Journal of Structural Engineering, Vol. 118 (4), pp. 1090-1105.

20. Ni, Y.Q., Hua X.G., Fan, K.Q., Ko, J.M. (2005), “Correlating Modal Properties with Temperature Using Long-term Monitoring Data and Support Vector Machine Technique”, Engineering Structures, Vol. 27, pp. 1762-1773.

21. Peeters, B., De Roeck., G. (2000), "One Year Monitoring of the Z24-Bridge: Environmental Influences Versus Damage Events”, Proceedings of IMAC XVIII, San Antonio, TX, pp. 1570-1576. 
22. Peeters, B., Maeck, J., De Roeck, G. (2001), "Vibration Based Damage Detection in Civil Engineering: Excitation Sources and Temperature Effects”, Smart Materials and Structures, Vol. 10, pp. 518-527.

23. Ruotolo, R., Surface, C. (1999), “Using SVD to Detect Damage in Structures with Different Operational Conditions”, Journal of Sound and Vibration, Vol. 226(3), pp. 425-439.

24. Rohrmann, R.G., Baessler, M., Said, S., Schmid, W., Ruecker, W.F. (2000), "Structural Causes of Temperature Affected Modal Data of Civil Structures Obtained by Long Time Monitoring", In Proceedings of IMAC-XVIII: A Conference on Structural Dynamics, San Antonio, Texas, USA, pp. 1-7.

25. Rahman, M., Lau, D.T. (2013), “A Comparative Study of System Identification Techniques Under Ambient Vibration", Proceedings of the 31st IMAC, A Conference on Structural Dynamics, Topics in Dynamics of Civil Structures, Vol. 9, pp. 145-154.

26. Sohn, H., Worden, K., Farrar C.R. (2003), "Statistical Damage Classification Under Changing Environment and Operational Conditions", Journal of Intelligent Material Systems and Structures, Vol. 13(9), pp. 561-574.

27. Sohn, H. (2007), "Effects of Environmental and Operational Variability on Structural Health Monitoring”, A Special Issue of Philosophical Transactions of the Royal Society on Structural Health Monitoring, Vol. 365, pp. 539-560.

28. Tehranian, A., Lau, D.T., Mills, S. (2002), "Pattern Recognition by Time Series Analysis of Monitoring Vibration Response of Bridges”, 5th European Conference on Structural Control, Genoa, Italy. pp. 1-8. 
29. Teughels, A., Maeck, J., De Roeck G. (2002), “Damage Assessment by FE Model Updating Using Damage Functions”, Computers and Structures, Vol. 80(25), pp. 18691879.

30. The Center for Engineering Strong Motion Data (CESMD) established by the US Geological Survey (USGS) and California Geological Survey (CGS).

31. Wood, M.G. (1992), "Damage Analysis of Bridge Structures Using Vibrational Technique", PhD Thesis, University of Aston, Birmingham, UK.

32. Westgate, R. J., Koo, K. Y., Brownjohn, J.M.W. (2011), "Environmental Effects on a Suspension Bridge's Dynamic Response", Proceedings of the 8th International Conference on Structural Dynamics, EURODYN, Leuven, Belgium, pp. 1208-1212.

33. Yan, A.M., Kerschen, G., De boe, P., Golinval, J.C. (2005a), "Damage Diagnosis Under Varying Environmental Conditions Part-I; A Linear Analysis", Journal of Mechanical Systems and Signal Processing, Vol. 19, pp. 845 -864.

34. Yan, A.M., Kerschen, G., De boe, P., Golinval, J.C. (2005b), "Structural Damage Diagnosis Under Varying Environmental Conditions-Part II: Local PCA for NonLinear Cases", Journal of Mechanical Systems and Signal Processing, Vol. 19, pp. 865 $-880$. 